ESAIM: M2AN 51 (2017) 225-278

DOI: $10.1051 / \mathrm{m} 2 \mathrm{an} / 2016020$
ESAIM: Mathematical Modelling and Numerical Analysis

www.esaim-m2an.org

\title{
NUMERICAL APPROXIMATION OF STOCHASTIC CONSERVATION LAWS ON BOUNDED DOMAINS
}

\author{
Caroline Bauzet $^{1}$, Julia Charrier ${ }^{2}$ and Thierry Gallouët ${ }^{2}$
}

\begin{abstract}
This paper is devoted to the study of finite volume methods for the discretization of scalar conservation laws with a multiplicative stochastic force defined on a bounded domain $D$ of $\mathbb{R}^{d}$ with Dirichlet boundary conditions and a given initial data in $L^{\infty}(D)$. We introduce a notion of stochastic entropy process solution which generalizes the concept of weak entropy solution introduced by F.Otto for such kind of hyperbolic bounded value problems in the deterministic case. Using a uniqueness result on this solution, we prove that the numerical solution converges to the unique stochastic entropy weak solution of the continuous problem under a stability condition on the time and space steps.
\end{abstract}

Mathematics Subject Classification. 35L60, 60H15, 35L60.

Received September 11, 2015. Accepted March 11, 2016.

\section{INTRODUCTION}

We wish to find an approximate solution to the following nonlinear scalar conservation law with a stochastic multiplicative force, posed over a bounded domain $D$ with initial condition and Dirichlet boundary conditions:

$$
\left\{\begin{aligned}
\mathrm{d} u+\operatorname{div}[\boldsymbol{v}(x, t) f(u)] \mathrm{d} t & =g(u) \mathrm{d} W \text { in } \Omega \times D \times(0, T), \\
u(\omega, x, 0) & =u_{0}(x), \quad \omega \in \Omega, x \in D, \\
u(\omega, x, t) & =u^{b}(x, t), \omega \in \Omega, x \in \partial D, t \in(0, T),
\end{aligned}\right.
$$

where $D \subset \mathbb{R}^{d}, d \in \mathbb{N}^{*}$ is a polygonal subset with boundary $\partial D, T>0$ and $W=\left\{W_{t}, \mathcal{F}_{t} ; 0 \leqslant t \leqslant T\right\}$ is a standard adapted one-dimensional continuous Brownian motion defined on the classical Wiener space $(\Omega, \mathcal{F}, P)$. In order to make the lecture more fluent, we omit in the sequel the variables $\omega, x, t$ and write $u$ instead of $u(\omega, x, t)$.

Note that, even in the deterministic case, a weak solution to a nonlinear scalar conservation law is not unique in general. The mathematical challenge consists in introducing a selection criterion in order to identify a unique solution. The notion of entropy solution was first introduced in the 70s by Kruzkhov in the case where the domain \footnotetext{
scheme, Dirichlet boundary conditions.

1 LMA, Aix-Marseille Univ, CNRS, UPR 7051, Centrale Marseille, 13402 Marseille cedex 20, France.

caroline.bauzet@univ-amu.fr

2 I2M, Aix-Marseille Univ, CNRS, UMR 7373, Centrale Marseille, 13453 Marseille, France.

julia.charrier; thierry.gallouet@univ-amu.fr
}

Keywords and phrases. Stochastic PDE, first-order hyperbolic equation, multiplicative noise, finite volume method, monotone 
was the whole space. In the present work we consider a stochastic version of the entropy condition proposed by Otto in his Ph.D. (see [19]) to take into account our non-homogeneous Dirichlet boundary conditions. We assume the following hypotheses:

$\mathrm{H}_{1}: u_{0} \in L^{\infty}(D)$.

$\mathrm{H}_{2}: u^{b} \in L^{\infty}(\partial D \times(0, T))$.

$\mathrm{H}_{3}: f: \mathbb{R} \rightarrow \mathbb{R}$ is a Lipschitz-continuous function with $f(0)=0$.

$\mathrm{H}_{4}: g: \mathbb{R} \rightarrow \mathbb{R}$ is a Lipschitz-continuous function.

$\mathrm{H}_{5}: \boldsymbol{v}: D \times[0, T] \rightarrow \mathbb{R}^{d}$ is a Lipschitz-continuous function and satisfies $\operatorname{div}[\boldsymbol{v}(x, t)]=0 \forall(x, t) \in D \times[0, T]$.

$\mathrm{H}_{6}$ : There exists $V<\infty$ such that $|\boldsymbol{v}(x, t)| \leqslant V \forall(x, t) \in D \times[0, T]$.

$\mathrm{H}_{7}: g$ is a bounded function.

Remark 1.1 (On these assumptions).

- $\mathrm{H}_{1}$ to $\mathrm{H}_{6}$ are used in the present work to prove the well-posedness of problem (1.1). Note that, as it is classically done for hyperbolic scalar conservation laws, for convenience one can assume that $f(0)=0$ without loss of generality.

- Note that the present study can be extended to the case $\operatorname{div}[\boldsymbol{v}(x, t)] \neq 0$ (which brings additional technical difficulties) following for example the work of [8] in the deterministic case.

$-\mathrm{H}_{7}$ is a technical and sufficient assumption used to show the convergence of the finite volume scheme (precisely to prove that the terms denoted $\tilde{C}^{h, k}-C^{h, k}$ and $\tilde{D}^{h, k}-D^{h, k}$ go to 0 in the proof of Prop. 5.3).

Remark 1.2. Note that we can also consider the case where $f$ is only locally Lipschitz-continuous if we make the additional assumption that $g$ has a compact support. Indeed in this case, by adapting the proof of Vallet [21] Section 6.1 , we can show that the stochastic entropy solution $u$ also belongs to $L^{\infty}(D)$. More precisely, thanks to the Itô formula, this maximum principle is direct for the viscous solution $u_{\epsilon}$, then it is conserved at the limit for $u$. Therefore it allows us to treat the cases where $f$ is only locally Lipschitz-continuous. In particular, all the results stated in this paper hold if one considers the stochastic Burgers equation (i.e. when $\left.f(u)=u^{2}\right)$.

\subsection{State of the art}

Only few papers have been devoted to the theoretical study of scalar conservation laws with a multiplicative stochastic forcing, let us mention in chronological order the contributions of $[3,6,7,9,11,14,15,17]$. The last of these papers is the only one which proposes to study the problem with nonhomogeneous Dirichlet boundary conditions, whereas in the other papers cited, the problem is studied on $\mathbb{R}^{d}$, on the torus or on bounded domain with homogeneous Dirichlet boundary conditions. Concerning the study of numerical approximation of these stochastic problems, there is also, to our knowledge, few papers. Let us cite the work of [16] and also its recent generalization to the multidimensional-case [2] where a time-discretization of the equation is proposed by the use of an operator-splitting method. Let us also mention the paper of [18] where a space-discretization of the equation is investigated by considering monotone numerical fluxes. In recent works $[4,5]$, proposed a time and space discretization of the problem in the case where the domain is the whole space $\mathbb{R}^{d}$ and showed the convergence of a class of flux-splitting finite volume scheme (in [4]) and more generally of monotone finite volume schemes (in [5]) towards the unique stochastic entropy solution of the problem by using the theoretical framework of [3]. For a thorough exposition of all these papers, we refer the reader to the introduction of [4].

\subsection{Goal of the study and outline of the paper}

The aim of this paper is to fill the gap left by the previous authors by proposing a both time and space discretization for multi-dimensional nonlinear scalar conservation laws forced by a multiplicative noise on a bounded domain with nonhomogeneous Dirichlet boundary conditions and studying the convergence of this scheme.

The paper is organized as follows. In Section 2, we propose the definition of a stochastic entropy solution for (1.1) and state the well-posedness result of the problem as a consequence of [17], which proposes a kinetic 
approach. In Section 3 we define the scheme used to approximate the stochastic entropy solution of (1.1). Then, we give the main result of this paper, which states the convergence of the approximate solution towards the unique stochastic entropy solution of the equation. The remainder of the paper is devoted to the proof of this convergence result. In Section 4, several preliminary results satisfied by the finite volume approximate solution denoted $u_{\mathcal{T}, k}$ are stated. Then, Section 5 is devoted to show the convergence of $u_{\mathcal{T}, k}$ towards the unique stochastic entropy solution of problem (1.1).

\subsection{General notations}

First of all, we need to introduce some notations and make precise the functional setting.

$-Q=D \times(0, T)$.

$-\mathbb{R}^{\star}=\mathbb{R} \backslash\{0\}$ and $\mathbb{N}^{\star}=\mathbb{N} \backslash\{0\}$.

- Throughout the paper, we denote by $C_{f}$ and $C_{g}$ the Lipschitz constants of $f$ and $g$.

- $|x|$ denotes the Euclidian norm of $x$ in $\mathbb{R}^{d}$ and $x . y$ the usual scalar product of $x$ and $y$ in $\mathbb{R}^{d}$.

- For $p=1, d$ or $d+1,\|.\|_{\infty}$ denotes the $L^{\infty}\left(\mathbb{R}^{p}\right)$ norm.

- E[--] denotes the expectation, i.e. the integral over $\Omega$ with respect to the probability measure $P$.

- $\mathcal{D}^{+}\left(\mathbb{R}^{d} \times[0, T)\right)$ denotes the subset of nonnegative elements of $\mathcal{D}\left(\mathbb{R}^{d} \times[0, T)\right)$.

- For a given separable Banach space $X$ we denote by $\mathcal{N}_{w}^{2}(0, T, X)$ the space of the predictable $X$-valued processes endowed with the norm $\|\phi\|_{\mathcal{N}_{w}^{2}(0, T, X)}^{2}:=E\left[\int_{0}^{T}\|\phi\|_{X}^{2} \mathrm{~d} t\right]$ (see Da Prato-Zabczyk [10]).

- $\mathcal{A}$ denotes the set of nonnegative convex functions $\eta$ in $C^{2,1}(\mathbb{R})$, such that $\eta$ admits 0 as a minimum, which is reached at a unique point $\kappa \in \mathbb{R}$. We also suppose that $\eta^{\prime}$ and $\eta^{\prime \prime}$ are bounded functions.

- $\Phi$ denotes the entropy flux defined for any $a \in \mathbb{R}$ and for any smooth function $\eta \in \mathcal{A}$ by $\Phi(a)=\int_{\kappa}^{a} \eta^{\prime}(\sigma) f^{\prime}(\sigma) \mathrm{d} \sigma$. Note in particular that $\Phi$ is a Lipschitz-continuous function.

\section{The Continuous problem}

Let us introduce in this section the definition of a solution for problem (1.1) and the existence and uniqueness result which ensures us the well-posedness of such a problem. This result is obtained under hypotheses $H_{1}$ to $H_{6}$. We follow [22], which establishs the convergence of finite volume monotone schemes for scalar conservation laws on bounded domains in the deterministic case. This work uses the concept of entropy solution introduced by Otto (see [19]) for Dirichlet boundary conditions. Such a notion of solution is well-suited for numerical approximation (see [22]) and is additionally equivalent to the BLN concept of solution in the case where the solution is of bounded variation. We adapt this notion of solution to the stochastic case.

Definition 2.1 (Stochastic entropy solution). A function $u$ of $\mathcal{N}_{w}^{2}\left(0, T, L^{2}(D)\right) \cap L^{\infty}\left(0, T, L^{2}(\Omega \times D)\right)$ is an entropy solution of the stochastic scalar conservation law (1.1) with the initial condition $u_{0} \in L^{\infty}(D)$, if P-a.s in $\Omega$, for any $\eta \in \mathcal{A}$ and for any $\varphi \in \mathcal{D}^{+}\left(\mathbb{R}^{d} \times[0, T)\right)$

$$
\begin{aligned}
0 \leqslant & \int_{D} \eta\left(u_{0}\right) \varphi(x, 0) \mathrm{d} x+C_{f} V \int_{0}^{T} \int_{\partial D} \varphi(x, t) \eta\left(u^{b}(x, t)\right) \mathrm{d} \gamma(x) \mathrm{d} t \\
& +\int_{Q} \eta(u) \partial_{t} \varphi(x, t) \mathrm{d} x \mathrm{~d} t+\int_{Q} \Phi(u) \boldsymbol{v}(x, t) . \nabla_{x} \varphi(x, t) \mathrm{d} x \mathrm{~d} t \\
& +\int_{0}^{T} \int_{D} \eta^{\prime}(u) g(u) \varphi(x, t) \mathrm{d} x \mathrm{~d} W(t)+\frac{1}{2} \int_{Q} g^{2}(u) \eta^{\prime \prime}(u) \varphi(x, t) \mathrm{d} x \mathrm{~d} t .
\end{aligned}
$$

For technical reasons, as in [5] for the case $D=\mathbb{R}^{n}$ and as in [22] for the deterministic case, we also need to consider a more general notion of solution. In fact, in a first step, we will only prove the convergence of the 
finite volume approximate solution $u_{\mathcal{T}, k}$ to a stochastic measure-valued entropy solution. Then, thanks to the result of uniqueness stated in Theorem 2.3, we will be able to deduce the convergence of $u_{\mathcal{T}, k}$ to the unique stochastic entropy solution of problem (1.1).

Definition 2.2 (Stochastic measure-valued entropy solution). A function $\mathbf{u}$ of $\mathcal{N}_{w}^{2}\left(0, T, L^{2}(D \times(0,1))\right) \cap$ $L^{\infty}\left(0, T, L^{2}(\Omega \times D \times(0,1))\right)$ is a measure-valued entropy solution of the stochastic scalar conservation law (1.1) with the initial condition $u_{0} \in L^{\infty}(D)$, if P-a.s in $\Omega$, for any $\eta \in \mathcal{A}$ and for any $\varphi \in \mathcal{D}^{+}\left(\mathbb{R}^{d} \times[0, T)\right)$

$$
\begin{aligned}
0 \leqslant & \int_{D} \eta\left(u_{0}\right) \varphi(x, 0) \mathrm{d} x+C_{f} V \int_{0}^{T} \int_{\partial D} \varphi(x, t) \eta\left(u^{b}(x, t)\right) \mathrm{d} \gamma(x) \mathrm{d} t \\
& +\int_{Q} \int_{0}^{1} \eta(\mathbf{u}(., \alpha)) \partial_{t} \varphi(x, t) \mathrm{d} \alpha \mathrm{d} x \mathrm{~d} t+\int_{Q} \int_{0}^{1} \Phi(\mathbf{u}(., \alpha)) \boldsymbol{v}(x, t) . \nabla_{x} \varphi(x, t) \mathrm{d} \alpha \mathrm{d} x \mathrm{~d} t \\
& +\int_{0}^{T} \int_{D} \int_{0}^{1} \eta^{\prime}(\mathbf{u}(., \alpha)) g(\mathbf{u}(., \alpha)) \varphi(x, t) \mathrm{d} \alpha \mathrm{d} x \mathrm{~d} W(t) \\
& +\frac{1}{2} \int_{Q} \int_{0}^{1} g^{2}(\mathbf{u}(., \alpha)) \eta^{\prime \prime}(\mathbf{u}(., \alpha)) \varphi(x, t) \mathrm{d} \alpha \mathrm{d} x \mathrm{~d} t .
\end{aligned}
$$

Theorem 2.3. Under assumptions $H_{1}$ to $H_{6}$ there exists a unique measure-valued entropy solution for problem (1.1). Moreover, it is the unique stochastic entropy solution in the sense of Definition 2.1.

Proof. According to the uniqueness and reduction result of [17], there exists a unique generalized kinetic solution which is actually a kinetic solution to the first order stochastic conservation law (1.1). Moreover, using the same arguments as in the work of [11], we can show that a kinetic solution is an entropy solution and vice versa. To conclude we just have to exploit the equivalence between the notions of measure-valued entropy solution and generalized kinetic solution.

\section{MAIN RESULT}

In the sequel, assume that assumptions $H_{1}$ to $H_{7}$ hold. Let us first give a definition of the admissible meshes for the finite volume scheme.

\subsection{Meshes and scheme}

Definition 3.1 (Admissible mesh). An admissible mesh $\mathcal{T}$ of $\mathbb{R}^{d}$ for the discretization of problem (1.1) is given by a family of disjoint connected polygonal subset of $D$ such that $\bar{D}$ is the union of the closure of the elements of $\mathcal{T}$ (which are called control volumes in the following) and such that the common interface of any two control volumes is included in a hyperplane of $\mathbb{R}^{d}$. It is assumed that $h=\operatorname{size}(\mathcal{T})=\sup \{\operatorname{diam}(K), K \in \mathcal{T}\}<\infty$ and that, for some $\bar{\alpha} \in \mathbb{R}_{+}^{*}$, we have

$$
\bar{\alpha} h^{d} \leqslant|K|, \quad \text { and } \quad|\partial K| \leqslant \frac{1}{\bar{\alpha}} h^{d-1}, \quad \forall K \in \mathcal{T},
$$

where we denote by

- $\partial K$ the boundary of the control volume $K$.

- $|K|$ the $d$-dimensional Lebesgue measure of $K$.

- $|\partial K|$ the $(d-1)$-dimensional Lebesgue measure of $\partial K$.

- $\mathcal{N}(K)$ the set of control volumes neighbors of the control volume $K$.

- $\sigma_{K, L}$ the common interface between $K$ and $L$ for any $L \in \mathcal{N}(K)$.

- $n_{K, L}$ the unit normal vector to interface $\sigma_{K, L}$, oriented from $K$ to $L$, for any $L \in \mathcal{N}(K)$.

- $\mathcal{E}$ the set of all the interfaces of the mesh $\mathcal{T}$. 
$-\mathcal{E}^{b}=\{\sigma \in \mathcal{E}:|\sigma \cap \partial D|>0\}$ the set of boundary interfaces.

- $\mathcal{E}_{K}$ the set of interfaces of the control volume $K$.

- $n_{K, \sigma}$ the unit normal to interface $\sigma$, outward to the control volume $K$, for any $\sigma \in \mathcal{E}_{K}$.

It follows easily from (3.1) the following inequality, which will be used several times later:

$$
\frac{|\partial K|}{|K|} \leqslant \frac{1}{\bar{\alpha}^{2} h}
$$

Remark 3.2. Since $|D|=\sum_{K \in \mathcal{T}}|K|$, assumption (3.1) yields the following estimate on the number of control volumes:

$$
\operatorname{Card}(\mathcal{T}) \leqslant \frac{|D|}{\bar{\alpha}} h^{-d}
$$

We now define the general monotone scheme. Consider an admissible mesh $\mathcal{T}$ in the sense of Definition 3.1. In order to compute an approximation of $u$ on $[0, T]$ we take $N \in \mathbb{N}^{\star}$ and define the time step $k=\frac{T}{N} \in \mathbb{R}_{+}^{\star}$. In this way $[0, T]=\bigcup_{n=0}^{N-1}[n k,(n+1) k]$.

The equations satisfied by the discrete unknowns denoted by $u_{K}^{n}, n \in\{0, \ldots, N-1\}, K \in \mathcal{T}$, are obtained by discretizing problem (1.1). For the discretization of such a problem, we need to define the numerical flux.

Definition 3.3 (Monotone numerical flux). We say that a function $F: \mathbb{R}^{2} \rightarrow \mathbb{R}$ is a monotone numerical flux if it satisfies the following properties:

- $F(a, b)$ is nondecreasing with respect to $a$ and nonincreasing with respect to $b$.

- There exists $F_{1}, F_{2}>0$ such that for any $a, b \in \mathbb{R}$ we have

$$
|F(b, a)-F(a, a)| \leqslant F_{1}|a-b| \text { and }|F(a, b)-F(a, a)| \leqslant F_{2}|a-b| .
$$

- $F(a, a)=f(a)$ for all $a \in \mathbb{R}$.

\section{Remark 3.4.}

- Note that it is not necessary to suppose $F$ to be continuous, even with respect to each variable separately.

- It is possible to choose a numerical flux $F$ depending on $\mathcal{T}, \sigma_{K, L}, n$, as soon as the constants $F_{1}, F_{2}$ can be chosen independently of $\mathcal{T}, \sigma_{K, L}, n$. For the sake of readability we will consider in what follows a numerical flux $F$ independent of $\mathcal{T}, \sigma_{K, L}, n$.

The set $\left\{u_{K}^{0}, K \in \mathcal{T}\right\}$ is given by the initial condition

$$
u_{K}^{0}=\frac{1}{|K|} \int_{K} u_{0}(x) \mathrm{d} x, \forall K \in \mathcal{T} .
$$

The equations satisfied by the discrete unknowns $u_{K}^{n}, n \in\{0, \ldots, N-1\}, K \in \mathcal{T}$ are given by the following explicit scheme associated to any monotone numerical flux $F$ : for any $K \in \mathcal{T}$, any $n \in\{0, \ldots, N-1\}$

$$
\frac{|K|}{k}\left(u_{K}^{n+1}-u_{K}^{n}\right)+\sum_{\sigma \in \mathcal{E}_{K}}|\sigma|\left\{v_{K, \sigma}^{n,+} F\left(u_{K}^{n}, u_{K, \sigma}^{n}\right)-v_{K, \sigma}^{n,-} F\left(u_{K, \sigma}^{n}, u_{K}^{n}\right)\right\}=|K| g\left(u_{K}^{n}\right) \frac{W^{n+1}-W^{n}}{k},
$$


where, by denoting $n_{K, \sigma}$ the unit normal vector to interface $\sigma \in \mathcal{E}_{K}$ outward to $K$ :

$$
\begin{aligned}
& v_{K, \sigma}^{n,+}=\frac{1}{k|\sigma|} \int_{n k}^{(n+1) k} \int_{\sigma}\left(\boldsymbol{v}(x, t) \cdot n_{K, \sigma}\right)^{+} \mathrm{d} \gamma(x) \mathrm{d} t, \\
& v_{K, \sigma}^{n,-}=\frac{1}{k|\sigma|} \int_{n k}^{(n+1) k} \int_{\sigma}\left(\boldsymbol{v}(x, t) \cdot n_{K, \sigma}\right)^{-} \mathrm{d} \gamma(x) \mathrm{d} t, \\
& u_{K, \sigma}^{n}= \begin{cases}u_{L}^{n} & \text { if } \sigma=\sigma_{K, L}, \\
u_{\sigma}^{b, n}=\frac{1}{k|\sigma|} \int_{n k}^{(n+1) k} \int_{\sigma} u^{b}(x, t) \mathrm{d} \gamma(x) \mathrm{d} t & \text { if } \sigma \in \mathcal{E}^{b},\end{cases} \\
& W^{n}=W(n k), \forall n \in\{0, \ldots, N-1\} .
\end{aligned}
$$

Remark 3.5. When $\sigma=\sigma_{K, L}$, we will denote $v_{K, L}^{n,+}=v_{K, \sigma_{K, L}}^{n,+}$ and $v_{K, L}^{n,-}=v_{K, \sigma_{K, L}}^{n,-}$, and using these notations, we have $v_{L, K}^{n,+}=v_{K, L}^{n,-}$.

The approximate finite volume solution $u_{\mathcal{T}, k}$ may be defined on $\Omega \times D \times[0, T)$ from the discrete unknowns $u_{K}^{n}$, $K \in \mathcal{T}, n \in\{0, \ldots, N-1\}$ which are computed in (3.6) by:

$$
u_{\mathcal{T}, k}(\omega, x, t)=u_{K}^{n} \text { for } \omega \in \Omega, x \in K \text { and } t \in[n k,(n+1) k) .
$$

Remark 3.6. Note that for any interface $\sigma \in \mathcal{E}$

$$
\begin{aligned}
v_{K, \sigma}^{n,+}-v_{K, \sigma}^{n,-} & =\frac{1}{k|\sigma|} \int_{n k}^{(n+1) k} \int_{\sigma} \boldsymbol{v}(x, t) \cdot n_{K, \sigma} \mathrm{d} \gamma(x) \mathrm{d} t \\
\text { and } v_{K, \sigma}^{n,+}+v_{K, \sigma}^{n,-}= & \frac{1}{k|\sigma|} \int_{n k}^{(n+1) k} \int_{\sigma}\left|\boldsymbol{v}(x, t) \cdot n_{K, \sigma}\right| \mathrm{d} \gamma(x) \mathrm{d} t .
\end{aligned}
$$

Moreover, $\operatorname{since} \operatorname{div}[\boldsymbol{v}(x, t)]=0$ for any $(x, t) \in D \times[0, T]$, we have

$$
\sum_{\sigma \in \mathcal{E}_{K}}|\sigma|\left(v_{K, \sigma}^{n,+}-v_{K, \sigma}^{n,-}\right)=0 .
$$

Indeed,

$$
\sum_{\sigma \in \mathcal{E}_{K}}|\sigma|\left(v_{K, \sigma}^{n,+}-v_{K, \sigma}^{n,-}\right)=\frac{1}{k} \int_{n k}^{(n+1) k} \sum_{\sigma \in \mathcal{E}_{K}} \int_{\sigma} \boldsymbol{v}(x, t) \cdot n_{K, \sigma} \mathrm{d} \gamma(x) \mathrm{d} t=\frac{1}{k} \int_{n k}^{(n+1) k} \int_{K} \operatorname{div}[\boldsymbol{v}(x, t)] \mathrm{d} x \mathrm{~d} t=0
$$

Remark 3.7. By denoting for any $\sigma \in \mathcal{E}, K \in \mathcal{T}$ and $n \in\{0, \ldots, N-1\}$

$$
F_{K, \sigma}^{n}(a, b)=|\sigma|\left\{v_{K, \sigma}^{n,+} F(a, b)-v_{K, \sigma}^{n,-} F(b, a)\right\},
$$

as a consequence of (3.8) we get that:

$$
\forall a \in \mathbb{R}, \forall K \in \mathcal{T}, \sum_{\sigma \in \mathcal{E}_{K}} F_{K, \sigma}^{n}(a, a)=0,
$$

which allows us to rewrite the numerical scheme (3.6) in the following way:

$$
\frac{|K|}{k}\left(u_{K}^{n+1}-u_{K}^{n}\right)+\sum_{\sigma \in \mathcal{E}_{K}}\left\{F_{K, \sigma}^{n}\left(u_{K}^{n}, u_{K, \sigma}^{n}\right)-F_{K, \sigma}^{n}\left(u_{K}^{n}, u_{K}^{n}\right)\right\}=|K| g\left(u_{K}^{n}\right) \frac{W^{n+1}-W^{n}}{k} .
$$


Remark 3.8 (On the measurability of the approximate finite volume solution). Let us mention that using properties of the Brownian motion, for all $K$ in $\mathcal{T}$ and all $n$ in $\{0, \ldots, N-1\}, u_{K}^{n}$ is $\mathcal{F}_{n k}$-measurable and so, as an elementary process adapted to the filtration $\left(\mathcal{F}_{t}\right)_{t \geqslant 0}, u_{\mathcal{T}, k}$ is predictable with values in $L^{2}(D)$.

\subsection{Main result}

We now state the main result of this paper.

Theorem 3.9 (Convergence to the stochastic entropy solution). Assume that hypotheses $H_{1}$ to $H_{7}$ hold. Let $\mathcal{T}$ be an admissible mesh in the sense of Definition $3.1, N \in \mathbb{N}^{\star}$ and $k=\frac{T}{N} \in \mathbb{R}_{+}^{\star}$ be the time step. Let $u_{\mathcal{T}, k}$ be the finite volume approximation defined by the monotone finite volume scheme (3.6) and (3.7). Then $u_{\mathcal{T}, k}$ converges to the unique stochastic entropy solution of (1.1) in the sense of Definition 2.1, in $L^{p}(\Omega \times Q)$ for any $p<2$ as $(h, k / h) \rightarrow(0,0)$.

Remark 3.10. Note that $u_{\mathcal{T}, k}$ converges particularly in $L p\left(\Omega, L^{p}(Q)\right)$ for any $1 \leqslant p<2$ and so in probability in the space of random variables with values in $L^{p}(Q)$.

Remark 3.11. Under the CFL Condition

$$
k \leqslant(1-\xi) \frac{\bar{\alpha}^{2} h}{V\left(F_{1}+F_{2}\right)}
$$

where $\bar{\alpha} \in \mathbb{R}_{+}^{\star}$ is a constant independent of the mesh coming from (3.1), we will prove in the sequel firstly for $\xi=0$, the $L_{t}^{\infty} L_{\omega, x}^{2}$ stability of $u_{\mathcal{T}, k}$ stated in Proposition 4.1, p.231, and secondly for some $\xi \in(0,1)$, the "weak BV" estimate stated in Proposition 4.3. In the deterministic case, condition (3.10) for some $\xi \in(0,1)$ is sufficient to show the convergence of $u_{\mathcal{T}, k}$ to the unique entropy solution of the problem, whereas in the stochastic case this condition doesn't seem to be sufficient, that is why we assume the stronger assumption $k / h \rightarrow 0$ as $h \rightarrow 0$. Note that this assumption on $k / h$ is perhaps technical and is quite weak (with respect to the usual CFL condition) since $k / h$ can goes to 0 as slowly as we want. An interesting point would be to see by using numerical simulations if it seems to be a necessary condition or not, but it is out of the scope of the present paper.

Remark 3.12. This theorem can easily be generalized to the case of a stochastic finite dimensional perturbation of the form $g(u) . d \mathbf{W}$ where $g$ takes values into $\mathbb{R}^{p}$ and $\mathbf{W}$ is a $p$-dimensional Brownian motion.

\section{Preliminary Results on the finite volume approximation}

Let us state in this section several results satisfied by the finite volume approximate solution $u_{\mathcal{T}, k}$ defined by (3.6) and (3.7).

\subsection{Stability estimates}

Proposition $4.1\left(L_{t}^{\infty} L_{\omega, x}^{2}\right.$ estimate). Let $T>0, u_{0} \in L^{2}(D), \mathcal{T}$ be an admissible mesh in the sense of Definition 3.1, $N \in \mathbb{N}^{\star}$ and $k=\frac{T}{N} \in \mathbb{R}_{+}^{\star}$ satisfying the Courant-Friedrichs-Levy (CFL) condition

$$
k \leqslant \frac{\bar{\alpha}^{2} h}{V\left(F_{1}+F_{2}\right)}
$$

Let $u_{\mathcal{T}, k}$ be the finite volume approximate solution defined by (3.6) and (3.7).

Then we have the following bound

$$
\left\|u_{\tau, k}\right\|_{L^{\infty}\left(0, T ; L^{2}(\Omega \times D)\right)} \leqslant C_{e s t}
$$


where

$$
C_{e s t}=\mathrm{e}^{C_{g}^{2} T}\left(\left\|u_{0}\right\|_{L^{2}(D)}^{2}+2 T|D| g^{2}(0)+V\left(F_{1}+F_{2}\right)\left\|u^{b}\right\|_{L^{2}((0, T) \times \partial D)}^{2}\right)^{1 / 2} .
$$

As a consequence we get

$$
\left\|u_{\mathcal{T}, k}\right\|_{L^{2}(\Omega \times Q)}^{2} \leqslant T C_{e s t}^{2} .
$$

Proof. First one has

$$
\begin{aligned}
\sum_{K \in \mathcal{T}}|K| E\left[\left(u_{K}^{0}\right)^{2}\right] & =\sum_{K \in \mathcal{T}}|K| E\left[\left(\frac{1}{|K|} \int_{K} u_{0}(x) \mathrm{d} x\right)^{2}\right] \\
& \leqslant\left\|u_{0}\right\|_{L^{2}(D)}^{2} .
\end{aligned}
$$

Set $n \in\{0, \ldots, N-1\}$. Let us multiply the numerical scheme (3.6) by $u_{K}^{n}$, we thus get

$$
\begin{aligned}
\frac{|K|}{k}\left[u_{K}^{n+1}-u_{K}^{n}\right] u_{K}^{n}= & -\sum_{\sigma \in \mathcal{E}_{K}}|\sigma|\left\{v_{K, \sigma}^{n,+} F\left(u_{K}^{n}, u_{K, \sigma}^{n}\right)-v_{K, \sigma}^{n,-} F\left(u_{K, \sigma}^{n}, u_{K}^{n}\right)\right\} u_{K}^{n} \\
& +\frac{|K|}{k} g\left(u_{K}^{n}\right)\left(W^{n+1}-W^{n}\right) u_{K}^{n} .
\end{aligned}
$$

And by using formula $a b=\frac{1}{2}\left[(a+b)^{2}-a^{2}-b^{2}\right]$ with $a=u_{K}^{n+1}-u_{K}^{n}$ and $b=u_{K}^{n}$ we obtain

$$
\begin{aligned}
\frac{1}{2} \frac{|K|}{k}\left[\left(u_{K}^{n+1}\right)^{2}-\left(u_{K}^{n}\right)^{2}-\left(u_{K}^{n+1}-u_{K}^{n}\right)^{2}\right]= & -\sum_{\sigma \in \mathcal{E}_{K}}|\sigma|\left\{v_{K, \sigma}^{n,+} F\left(u_{K}^{n}, u_{K, \sigma}^{n}\right)-v_{K, \sigma}^{n,-} F\left(u_{K, \sigma}^{n}, u_{K}^{n}\right)\right\} u_{K}^{n} \\
& +\frac{|K|}{k} g\left(u_{K}^{n}\right)\left(W^{n+1}-W^{n}\right) u_{K}^{n},
\end{aligned}
$$

and then

$$
\begin{aligned}
\frac{|K|}{2}\left[\left(u_{K}^{n+1}\right)^{2}-\left(u_{K}^{n}\right)^{2}\right]= & \frac{|K|}{2}\left(u_{K}^{n+1}-u_{K}^{n}\right)^{2}-k \sum_{\sigma \in \mathcal{E}_{K}}|\sigma|\left\{v_{K, \sigma}^{n,+} F\left(u_{K}^{n}, u_{K, \sigma}^{n}\right)-v_{K, \sigma}^{n,-} F\left(u_{K, \sigma}^{n}, u_{K}^{n}\right)\right\} u_{K}^{n} \\
& +|K| g\left(u_{K}^{n}\right)\left(W^{n+1}-W^{n}\right) u_{K}^{n} .
\end{aligned}
$$

Using the finite volume scheme (3.6) we can replace $\left(u_{K}^{n+1}-u_{K}^{n}\right)^{2}$ and we take then the expectation. Thanks to the independance between the random variables $\left(W^{n+1}-W^{n}\right)$ and $u_{K}^{n}$, together with the equality

we get

$$
E\left[\left(g\left(u_{K}^{n}\right)\left(W^{n+1}-W^{n}\right)\right)^{2}\right]=E\left[\left(g\left(u_{K}^{n}\right)\right)^{2}\right] E\left[\left(W^{n+1}-W^{n}\right)^{2}\right]=k E\left[\left(g\left(u_{K}^{n}\right)\right)^{2}\right],
$$

$$
\begin{aligned}
& \frac{|K|}{2} E\left[\left(u_{K}^{n+1}\right)^{2}-\left(u_{K}^{n}\right)^{2}\right] \\
&=\frac{|K|}{2} E\left[\left(-\frac{k}{|K|} \sum_{\sigma \in \mathcal{E}_{K}}|\sigma|\left\{v_{K, \sigma}^{n,+} F\left(u_{K}^{n}, u_{K, \sigma}^{n}\right)-v_{K, \sigma}^{n,-} F\left(u_{K, \sigma}^{n}, u_{K}^{n}\right)\right\}+g\left(u_{K}^{n}\right)\left(W^{n+1}-W^{n}\right)\right)^{2}\right] \\
&-k E\left[\sum_{\sigma \in \mathcal{E}_{K}}|\sigma|\left\{v_{K, \sigma}^{n,+} F\left(u_{K}^{n}, u_{K, \sigma}^{n}\right)-v_{K, \sigma}^{n,-} F\left(u_{K, \sigma}^{n}, u_{K}^{n}\right)\right\} u_{K}^{n}\right]+|K| E\left[g\left(u_{K}^{n}\right)\left(W^{n+1}-W^{n}\right) u_{K}^{n}\right] \\
&= \frac{k^{2}}{2|K|} E\left[\left(\sum_{\sigma \in \mathcal{E}_{K}}|\sigma|\left\{v_{K, \sigma}^{n,+} F\left(u_{K}^{n}, u_{K, \sigma}^{n}\right)-v_{K, \sigma}^{n,-} F\left(u_{K, \sigma}^{n}, u_{K}^{n}\right)\right\}\right)^{2}\right]+\frac{k|K|}{2} E\left[\left(g\left(u_{K}^{n}\right)\right)^{2}\right] \\
&-k E\left[\sum_{\sigma \in \mathcal{E}_{K}}|\sigma|\left\{v_{K, \sigma}^{n,+} F\left(u_{K}^{n}, u_{K, \sigma}^{n}\right)-v_{K, \sigma}^{n,-} F\left(u_{K, \sigma}^{n}, u_{K}^{n}\right)\right\} u_{K}^{n}\right] .
\end{aligned}
$$


Using (3.8), which states that $\sum_{\sigma \in \mathcal{E}_{K}}|\sigma|\left(v_{K, \sigma}^{n,+}-v_{K, \sigma}^{n,-}\right)=0$, this equality can be rewritten as, after summing over each control volume $K \in \mathcal{T}$,

$$
\sum_{K \in \mathcal{T}} \frac{|K|}{2} E\left[\left(u_{K}^{n+1}\right)^{2}-\left(u_{K}^{n}\right)^{2}\right]=B_{1}-B_{2}+B_{3},
$$

where

$$
\begin{aligned}
& B_{1}=\sum_{K \in \mathcal{T}} \frac{k^{2}}{2|K|} E\left[\left(\sum_{\sigma \in \mathcal{E}_{K}}|\sigma|\left\{v_{K, \sigma}^{n,+}\left(F\left(u_{K}^{n}, u_{K, \sigma}^{n}\right)-f\left(u_{K}^{n}\right)\right)-v_{K, \sigma}^{n,-}\left(F\left(u_{K, \sigma}^{n}, u_{K}^{n}\right)-f\left(u_{K}^{n}\right)\right)\right\}\right)^{2}\right] \\
& B_{2}=\sum_{K \in \mathcal{T}} k E\left[\sum_{\sigma \in \mathcal{E}_{K}}|\sigma|\left\{v_{K, \sigma}^{n,+}\left(F\left(u_{K}^{n}, u_{K, \sigma}^{n}\right)-f\left(u_{K}^{n}\right)\right)-v_{K, \sigma}^{n,-}\left(F\left(u_{K, \sigma}^{n}, u_{K}^{n}\right)-f\left(u_{K}^{n}\right)\right)\right\} u_{K}^{n}\right]
\end{aligned}
$$

and

$$
B_{3}=\sum_{K \in \mathcal{T}} \frac{k|K|}{2} E\left[\left(g\left(u_{K}^{n}\right)\right)^{2}\right] .
$$

- Study of $B_{1}$ : Using the notations

$$
\begin{gathered}
A=F\left(u_{K}^{n}, u_{K, \sigma}^{n}\right)-f\left(u_{K}^{n}\right), B=F\left(u_{K, \sigma}^{n}, u_{K}^{n}\right)-f\left(u_{K}^{n}\right) \\
\zeta=\frac{v_{K, \sigma}^{n,+}}{v_{K, \sigma}^{n,+}+v_{K, \sigma}^{n,-}}, \quad \text { and } \quad 1-\zeta=\frac{v_{K, \sigma}^{n,-}}{v_{K, \sigma}^{n,+}+v_{K, \sigma}^{n,-}},
\end{gathered}
$$

since $\zeta \in(0,1)$ we get using Cauchy-Schwarz inequality that

$$
\begin{aligned}
\left(\sum_{\sigma \in \mathcal{E}_{K}}|\sigma|\left\{v_{K, \sigma}^{n,+} A-v_{K, \sigma}^{n,-} B\right\}\right)^{2} & =\left(\sum_{\sigma \in \mathcal{E}_{K}}|\sigma|\left(v_{K, \sigma}^{n,+}+v_{K, \sigma}^{n,-}\right)\{\zeta A-(1-\zeta) B\}\right)^{2} \\
& \leqslant \sum_{\sigma \in \mathcal{E}_{K}}|\sigma|\left(v_{K, \sigma}^{n,+}+v_{K, \sigma}^{n,-}\right) \sum_{\sigma \in \mathcal{E}_{K}}|\sigma|\left(v_{K, \sigma}^{n,+}+v_{K, \sigma}^{n,-}\right)\{\zeta A+(1-\zeta)(-B)\}^{2} \\
& \leqslant \sum_{\sigma \in \mathcal{E}_{K}}|\sigma|\left(v_{K, \sigma}^{n,+}+v_{K, \sigma}^{n,-}\right) \sum_{\sigma \in \mathcal{E}_{K}}|\sigma|\left(v_{K, \sigma}^{n,+}+v_{K, \sigma}^{n,-}\right)\left\{\zeta A^{2}+(1-\zeta) B^{2}\right\} .
\end{aligned}
$$

Since $\left(v_{K, \sigma}^{n,+}+v_{K, \sigma}^{n,-}\right) \zeta=v_{K, \sigma}^{n,+}$ and $\left(v_{K, \sigma}^{n,+}+v_{K, \sigma}^{n,-}\right)(1-\zeta)=v_{K, \sigma}^{n,-}$, we get the following estimate

$$
\begin{aligned}
B_{1} \leqslant & \sum_{K \in \mathcal{T}} \frac{k^{2}}{2|K|}\left(\sum_{\sigma \in \mathcal{E}_{K}}|\sigma|\left(v_{K, \sigma}^{n,+}+v_{K, \sigma}^{n,-}\right)\right) \times E\left[\sum _ { \sigma \in \mathcal { E } _ { K } } | \sigma | \left\{v_{K, \sigma}^{n,+}\left(F\left(u_{K}^{n}, u_{K, \sigma}^{n}\right)-f\left(u_{K}^{n}\right)\right)^{2}\right.\right. \\
& \left.\left.+v_{K, \sigma}^{n,-}\left(F\left(u_{K, \sigma}^{n}, u_{K}^{n}\right)-f\left(u_{K}^{n}\right)\right)^{2}\right\}\right] .
\end{aligned}
$$

Using the fact that

$$
\sum_{\sigma \in \mathcal{E}_{K}}|\sigma|\left(v_{K, \sigma}^{n,+}+v_{K, \sigma}^{n,-}\right) \leqslant V|\partial K|
$$


which implies thanks to (3.2) and to the CFL Condition (4.1) that

$$
\frac{k}{|K|} \sum_{\sigma \in \mathcal{E}_{K}}|\sigma|\left(v_{K, \sigma}^{n,+}+v_{K, \sigma}^{n,-}\right) \leqslant k V \frac{|\partial K|}{|K|} \leqslant \frac{\bar{\alpha}^{2} h}{V\left(F_{1}+F_{2}\right)} V \frac{1}{\bar{\alpha}^{2} h}=\frac{1}{F_{1}+F_{2}},
$$

we have

$$
B_{1} \leqslant \frac{k}{2\left(F_{1}+F_{2}\right)} \sum_{K \in \mathcal{T}} \sum_{\sigma \in \mathcal{E}_{K}}|\sigma| E\left[v_{K, \sigma}^{n,+}\left(F\left(u_{K}^{n}, u_{K, \sigma}^{n}\right)-f\left(u_{K}^{n}\right)\right)^{2}+v_{K, \sigma}^{n,-}\left(F\left(u_{K, \sigma}^{n}, u_{K}^{n}\right)-f\left(u_{K}^{n}\right)\right)^{2}\right] .
$$

By denoting

$$
\mathfrak{T}_{n}=\left\{(K, L) \in \mathcal{T}^{2}: L \in \mathcal{N}(K) \text { and } u_{K}^{n}>u_{L}^{n}\right\},
$$

we see that the double sum in the right hand side of (4.6) can be gathered by edges, according to the following formula (see [22], Lem. 7, p. 582):

$$
\sum_{K \in \mathcal{T}} \sum_{\sigma \in \mathcal{E}_{K}} \rho_{K, \sigma}^{n}=\sum_{\sigma \in \mathcal{E}^{b}} \rho_{K, \sigma}^{n}+\sum_{(K, L) \in \mathfrak{T}_{n}}\left(\rho_{K, K \mid L}^{n}+\rho_{L, K \mid L}^{n}\right),
$$

where $\rho_{K, \sigma}^{n}=|\sigma| E\left[v_{K, \sigma}^{n,+}\left(F\left(u_{K}^{n}, u_{K, \sigma}^{n}\right)-f\left(u_{K}^{n}\right)\right)^{2}+v_{K, \sigma}^{n,-}\left(F\left(u_{K, \sigma}^{n}, u_{K}^{n}\right)-f\left(u_{K}^{n}\right)\right)^{2}\right]$. This finally gives:

$$
B_{1} \leqslant B_{1,1}+B_{1,2}
$$

where

$$
\begin{aligned}
B_{1,1}= & \frac{k}{2\left(F_{1}+F_{2}\right)} \sum_{\sigma \in \mathcal{E}^{b}}|\sigma| E\left[v_{K, \sigma}^{n,+}\left(F\left(u_{K}^{n}, u_{\sigma}^{b, n}\right)-f\left(u_{K}^{n}\right)\right)^{2}+v_{K, \sigma}^{n,-}\left(F\left(u_{\sigma}^{b, n}, u_{K}^{n}\right)-f\left(u_{K}^{n}\right)\right)^{2}\right] \\
B_{1,2}= & \frac{k}{2\left(F_{1}+F_{2}\right)} \sum_{(K, L) \in \mathfrak{T}_{n}}\left|\sigma_{K, L}\right| E\left[v_{K, L}^{n,+}\left\{\left(F\left(u_{K}^{n}, u_{L}^{n}\right)-f\left(u_{K}^{n}\right)\right)^{2}+\left(F\left(u_{K}^{n}, u_{L}^{n}\right)-f\left(u_{L}^{n}\right)\right)^{2}\right\}\right. \\
& \left.+v_{K, L}^{n,-}\left\{\left(f\left(u_{K}^{n}\right)-F\left(u_{L}^{n}, u_{K}^{n}\right)\right)^{2}+\left(f\left(u_{L}^{n}\right)-F\left(u_{L}^{n}, u_{K}^{n}\right)\right)^{2}\right\}\right] .
\end{aligned}
$$

- Study of $B_{2}$ : We introduce the term $B_{2,1}$ defined by

$$
B_{2,1}=k \sum_{\sigma \in \mathcal{E}^{b}}|\sigma| E\left[u_{K}^{n}\left\{v_{K, \sigma}^{n,+}\left(F\left(u_{K}^{n}, u_{\sigma}^{b, n}\right)-f\left(u_{K}^{n}\right)\right)-v_{K, \sigma}^{n,-}\left(F\left(u_{\sigma}^{b, n}, u_{K}^{n}\right)-f\left(u_{K}^{n}\right)\right)\right\}\right] .
$$

We have then

$$
\begin{aligned}
B_{2}-B_{2,1}= & k \sum_{(K, L) \in \mathfrak{T}_{n}}\left|\sigma_{K, L}\right| E\left[v_{K, L}^{n,+}\left\{u_{K}^{n}\left(F\left(u_{K}^{n}, u_{L}^{n}\right)-f\left(u_{K}^{n}\right)\right)-u_{L}^{n}\left(F\left(u_{K}^{n}, u_{L}^{n}\right)-f\left(u_{L}^{n}\right)\right)\right\}\right. \\
& \left.-v_{K, L}^{n,-}\left\{u_{K}^{n}\left(F\left(u_{L}^{n}, u_{K}^{n}\right)-f\left(u_{K}^{n}\right)\right)-u_{L}^{n}\left(F\left(u_{L}^{n}, u_{K}^{n}\right)-f\left(u_{L}^{n}\right)\right)\right\}\right] .
\end{aligned}
$$

Denoting by $\phi$ the function defined for any $a \in \mathbb{R}$ by $\phi(a)=\int_{0}^{a} s f^{\prime}(s) \mathrm{d} s$, an integration by parts yields, for all $(a, b) \in \mathbb{R}^{2}$

$$
\phi(b)-\phi(a)=\int_{a}^{b} s f^{\prime}(s) \mathrm{d} s=b(f(b)-F(a, b))-a(f(a)-F(a, b))-\int_{a}^{b}(f(s)-F(a, b)) \mathrm{d} s .
$$


Using this formula, we define $B_{2,2}$ and $B_{2,3}$ by

$$
B_{2,2}=E\left[\sum_{(K, L) \in \mathfrak{T}_{n}} k\left|\sigma_{K, L}\right|\left\{v_{K, L}^{n,+}\left(\int_{u_{K}^{n}}^{u_{L}^{n}}\left(f(s)-F\left(u_{K}^{n}, u_{L}^{n}\right)\right) \mathrm{d} s\right)+v_{K, L}^{n,-}\left(\int_{u_{L}^{n}}^{u_{K}^{n}}\left(f(s)-F\left(u_{L}^{n}, u_{K}^{n}\right)\right) \mathrm{d} s\right)\right\}\right]
$$

and

$$
B_{2,3}=-E\left[\sum_{(K, L) \in \mathfrak{T}_{n}} k\left|\sigma_{K, L}\right|\left(v_{K, L}^{n,+}-v_{K, L}^{n,-}\right)\left\{\phi\left(u_{K}^{n}\right)-\phi\left(u_{L}^{n}\right)\right\}\right] .
$$

We have then split $B_{2}$ into three terms:

$$
B_{2}=B_{2,1}+B_{2,2}+B_{2,3} .
$$

Note that since $\operatorname{div}[\boldsymbol{v}(x, t)]=0 \forall(x, t) \in D \times[0, T]$, one has

$$
\begin{aligned}
B_{2,3}= & -E\left[\sum_{(K, L) \in \mathfrak{T}_{n}} k\left|\sigma_{K, L}\right|\left(\frac{1}{k\left|\sigma_{K, L}\right|} \int_{n k}^{(n+1) k} \int_{\sigma_{K, L}} \boldsymbol{v}(x, t) \cdot n_{K, L} \mathrm{~d} \gamma(x) \mathrm{d} t\right)\left\{\phi\left(u_{K}^{n}\right)-\phi\left(u_{L}^{n}\right)\right\}\right] \\
= & -E\left[\sum _ { ( K , L ) \in \mathfrak { T } _ { n } } \left\{\left(\int_{n k}^{(n+1) k} \int_{\sigma_{K, L}} \boldsymbol{v}(x, t) \cdot n_{K, L} \mathrm{~d} \gamma(x) \mathrm{d} t\right) \phi\left(u_{K}^{n}\right)\right.\right. \\
& \left.\left.+\left[\int_{n k}^{(n+1) k} \int_{\sigma_{K, L}} \boldsymbol{v}(x, t) \cdot n_{L, K} \mathrm{~d} \gamma(x) \mathrm{d} t\right) \phi\left(u_{L}^{n}\right)\right\}\right] \\
=E & {\left[\sum_{\sigma \in \mathcal{E}^{b}} k|\sigma|\left(v_{K, \sigma}^{n,+}-v_{K, \sigma}^{n,-}\right) \phi\left(u_{K}^{n}\right)\right]-E\left[\sum_{K \in \mathcal{T}} \sum_{\sigma \in \mathcal{E}_{K}} \phi\left(u_{K}^{n}\right) \int_{n k}^{(n+1) k} \int_{\sigma_{K, L}} \boldsymbol{v}(x, t) \cdot n_{K, L} \mathrm{~d} \gamma(x) \mathrm{d} t\right] } \\
=E & {\left[\sum_{\sigma \in \mathcal{E}^{b}} k|\sigma|\left(v_{K, \sigma}^{n,+}-v_{K, \sigma}^{n,-}\right) \phi\left(u_{K}^{n}\right)\right]-E\left[\sum_{K \in \mathcal{T}} \phi\left(u_{K}^{n}\right) \int_{n k}^{(n+1) k} \int_{K} \operatorname{div}[\boldsymbol{v}(x, t)] \mathrm{d} x \mathrm{~d} t\right] } \\
=E & {\left[\sum_{\sigma \in \mathcal{E}^{b}} k|\sigma|\left(v_{K, \sigma}^{n,+}-v_{K, \sigma}^{n,-}\right) \phi\left(u_{K}^{n}\right)\right] . }
\end{aligned}
$$

Next, we will estimate simultaneously $B_{2,1}$ and $B_{2,3}$ which correspond to the terms on the boundary of the domain. To do this, we first introduce the following technical lemma from [13] (Lem. 4.5, p. 107), which will be used several times in the sequel:

Lemma 4.2. Let $\mathcal{G}: \mathbb{R} \rightarrow \mathbb{R}$ be a monotone Lipschitz-continuous function with a Lipschitz constant $C_{\mathcal{G}}>0$. Then:

$$
\left|\int_{c}^{d} \mathcal{G}(t)-\mathcal{G}(c) \mathrm{d} t\right| \geqslant \frac{1}{2 C_{\mathcal{G}}}(\mathcal{G}(d)-\mathcal{G}(c))^{2}, \forall c, d \in \mathbb{R}
$$

Thanks to this lemma, we estimate $B_{2,1}$ by treating separately the terms $u_{K}^{n}\left(F\left(u_{K}^{n}, u_{\sigma}^{b, n}\right)-f\left(u_{K}^{n}\right)\right)$ and $-u_{K}^{n}\left(F\left(u_{\sigma}^{b, n}, u_{K}^{n}\right)-f\left(u_{K}^{n}\right)\right)$ :

- Study of $u_{K}^{n}\left(F\left(u_{K}^{n}, u_{\sigma}^{b, n}\right)-f\left(u_{K}^{n}\right)\right)$ : by using the nonincreasing and $F_{2}$-Lispchitz continuous function $\varphi_{d}$ function defined by

$$
\varphi_{d}(s)=F\left(u_{K}^{n}, s\right), \forall s \in \mathbb{R},
$$


we have

$$
u_{K}^{n}\left(F\left(u_{K}^{n}, u_{\sigma}^{b, n}\right)-f\left(u_{K}^{n}\right)\right)=u_{K}^{n}\left(F\left(u_{K}^{n}, u_{\sigma}^{b, n}\right)-F\left(u_{K}^{n}, u_{K}^{n}\right)\right)=u_{K}^{n}\left(\varphi_{d}\left(u_{\sigma}^{b, n}\right)-\varphi_{d}\left(u_{K}^{n}\right)\right) .
$$

We now introduce the function $\phi_{d}$ defined by $\phi_{d}(a)=a \varphi_{d}(a)-\int_{0}^{a} \varphi_{d}(s) \mathrm{d} s$ for any $a \in \mathbb{R}$, one has then for any $a, b \in \mathbb{R}$ :

$$
\phi_{d}(b)-\phi_{d}(a)=b\left(\varphi_{d}(b)-\varphi_{d}(a)\right)-\int_{a}^{b} \varphi_{d}(s)-\varphi_{d}(a) \mathrm{d} s
$$

With $a=u_{\sigma}^{b, n}$ and $b=u_{K}^{n}$, we deduce from this last equality that

$$
\begin{aligned}
u_{K}^{n}\left(F\left(u_{K}^{n}, u_{\sigma}^{b, n}\right)-f\left(u_{K}^{n}\right)\right) & =-u_{K}^{n}\left(\varphi_{d}\left(u_{K}^{n}\right)-\varphi_{d}\left(u_{\sigma}^{b, n}\right)\right) \\
& =\phi_{d}\left(u_{\sigma}^{b, n}\right)-\phi_{d}\left(u_{K}^{n}\right)-\int_{u_{\sigma}^{b, n}}^{u_{K}^{n}} \varphi_{d}(s)-\varphi_{d}\left(u_{\sigma}^{b, n}\right) \mathrm{d} s \\
& \geqslant \phi_{d}\left(u_{\sigma}^{b, n}\right)-\phi_{d}\left(u_{K}^{n}\right)+\frac{1}{2 F_{2}}\left(\varphi_{d}\left(u_{\sigma}^{b, n}\right)-\varphi_{d}\left(u_{K}^{n}\right)\right)^{2} \\
& \geqslant \phi_{d}\left(u_{\sigma}^{b, n}\right)-\phi_{d}\left(u_{K}^{n}\right)+\frac{1}{2\left(F_{1}+F_{2}\right)}\left(F\left(u_{K}^{n}, u_{\sigma}^{b, n}\right)-f\left(u_{K}^{n}\right)\right)^{2} .
\end{aligned}
$$

- Study of $-u_{K}^{n}\left(F\left(u_{\sigma}^{b, n}, u_{K}^{n}\right)-f\left(u_{K}^{n}\right)\right)$ : by using the nondecreasing and $F_{1}$-Lispchitz continuous function $\varphi_{g}$ defined by

$$
\varphi_{g}(s)=F\left(s, u_{K}^{n}\right), \forall s \in \mathbb{R}
$$

we have

$$
-u_{K}^{n}\left(F\left(u_{\sigma}^{b, n}, u_{K}^{n}\right)-f\left(u_{K}^{n}\right)\right)=u_{K}^{n}\left(F\left(u_{K}^{n}, u_{K}^{n}\right)-F\left(u_{\sigma}^{b, n}, u_{K}^{n}\right)\right)=u_{K}^{n}\left(\varphi_{g}\left(u_{K}^{n}\right)-\varphi_{g}\left(u_{\sigma}^{b, n}\right)\right) .
$$

We now introduce the function $\phi_{g}$ defined by $\phi_{g}(a)=a \varphi_{g}(a)-\int_{0}^{a} \varphi_{g}(s) \mathrm{d} s$ for any $a \in \mathbb{R}$, one has then for any $a, b \in \mathbb{R}$ :

$$
\phi_{g}(b)-\phi_{g}(a)=b\left(\varphi_{g}(b)-\varphi_{g}(a)\right)-\int_{a}^{b} \varphi_{g}(s)-\varphi_{g}(a) \mathrm{d} s
$$

With $a=u_{\sigma}^{b, n}$ and $b=u_{K}^{n}$, we deduce from this last equality that

$$
\begin{aligned}
-u_{K}^{n}\left(F\left(u_{\sigma}^{b, n}, u_{K}^{n}\right)-f\left(u_{K}^{n}\right)\right) & =u_{K}^{n}\left(\varphi_{g}\left(u_{K}^{n}\right)-\varphi_{g}\left(u_{\sigma}^{b, n}\right)\right) \\
& =\phi_{g}\left(u_{K}^{n}\right)-\phi_{g}\left(u_{\sigma}^{b, n}\right)+\int_{u_{\sigma}^{b, n}}^{u_{K}^{n}} \varphi_{g}(s)-\varphi_{g}\left(u_{\sigma}^{b, n}\right) \mathrm{d} s \\
& \geqslant \phi_{g}\left(u_{K}^{n}\right)-\phi_{g}\left(u_{\sigma}^{b, n}\right)+\frac{1}{2 F_{1}}\left(F\left(u_{\sigma}^{b, n}, u_{K}^{n}\right)-f\left(u_{K}^{n}\right)\right)^{2} \\
& \geqslant \phi_{g}\left(u_{K}^{n}\right)-\phi_{g}\left(u_{\sigma}^{b, n}\right)+\frac{1}{2\left(F_{1}+F_{2}\right)}\left(F\left(u_{\sigma}^{b, n}, u_{K}^{n}\right)-f\left(u_{K}^{n}\right)\right)^{2} .
\end{aligned}
$$


- Thanks to (4.9) and (4.10) we get

$$
\begin{aligned}
B_{2,1}= & k \sum_{\sigma \in \mathcal{E}^{b}}|\sigma| E\left[u_{K}^{n}\left\{v_{K, \sigma}^{n,+}\left(F\left(u_{K}^{n}, u_{\sigma}^{b, n}\right)-f\left(u_{K}^{n}\right)\right)-v_{K, \sigma}^{n,-}\left(F\left(u_{\sigma}^{b, n}, u_{K}^{n}\right)-f\left(u_{K}^{n}\right)\right)\right\}\right] \\
\geqslant & k \sum_{\sigma \in \mathcal{E}^{b}}|\sigma| E\left[v_{K, \sigma}^{n,+}\left\{\phi_{d}\left(u_{\sigma}^{b, n}\right)-\phi_{d}\left(u_{K}^{n}\right)+\frac{1}{2\left(F_{1}+F_{2}\right)}\left(F\left(u_{K}^{n}, u_{\sigma}^{b, n}\right)-f\left(u_{K}^{n}\right)\right)^{2}\right\}\right. \\
& \left.+v_{K, \sigma}^{n,-}\left\{\phi_{g}\left(u_{K}^{n}\right)-\phi_{g}\left(u_{\sigma}^{b, n}\right)+\frac{1}{2\left(F_{1}+F_{2}\right)}\left(F\left(u_{\sigma}^{b, n}, u_{K}^{n}\right)-f\left(u_{K}^{n}\right)\right)^{2}\right\}\right] \\
= & \frac{k}{2\left(F_{1}+F_{2}\right)} \sum_{\sigma \in \mathcal{E}^{b}}|\sigma| E\left[v_{K, \sigma}^{n,+}\left(F\left(u_{K}^{n}, u_{\sigma}^{b, n}\right)-f\left(u_{K}^{n}\right)\right)^{2}+v_{K, \sigma}^{n,-}\left(F\left(u_{\sigma}^{b, n}, u_{K}^{n}\right)-f\left(u_{K}^{n}\right)\right)^{2}\right] \\
& +k \sum_{\sigma \in \mathcal{E}^{b}}|\sigma| E\left[v_{K, \sigma}^{n,+}\left\{\phi_{d}\left(u_{\sigma}^{b, n}\right)-\phi_{d}\left(u_{K}^{n}\right)\right\}+v_{K, \sigma}^{n,-}\left\{\phi_{g}\left(u_{K}^{n}\right)-\phi_{g}\left(u_{\sigma}^{b, n}\right)\right\}\right] \\
= & B_{1,1}+k \sum_{\sigma \in \mathcal{E}^{b}}|\sigma| E\left[v_{K, \sigma}^{n,+}\left\{\phi_{d}\left(u_{\sigma}^{b, n}\right)-\phi_{d}\left(u_{K}^{n}\right)\right\}+v_{K, \sigma}^{n,-}\left\{\phi_{g}\left(u_{K}^{n}\right)-\phi_{g}\left(u_{\sigma}^{b, n}\right)\right\}\right] \\
\geqslant & B_{1,1}+k \sum_{\sigma \in \mathcal{E}^{b}}|\sigma| E\left[v_{K, \sigma}^{n,-} \phi_{g}\left(u_{K}^{n}\right)-v_{K, \sigma}^{n,+} \phi_{d}\left(u_{K}^{n}\right)\right]+k \sum_{\sigma \in \mathcal{E}^{b}}|\sigma| E\left[v_{K, \sigma}^{n,+} \phi_{d}\left(u_{\sigma}^{b, n}\right)-v_{K, \sigma}^{n,-} \phi_{g}\left(u_{\sigma}^{b, n}\right)\right]
\end{aligned}
$$

- Let us now estimate $B_{2,1}+B_{2,3}$ :

$$
\begin{aligned}
B_{2,1}+B_{2,3} \geqslant & B_{1,1}+k \sum_{\sigma \in \mathcal{E}^{b}}|\sigma| E\left[v_{K, \sigma}^{n,-} \phi_{g}\left(u_{K}^{n}\right)-v_{K, \sigma}^{n,+} \phi_{d}\left(u_{K}^{n}\right)\right]-k \sum_{\sigma \in \mathcal{E}^{b}}|\sigma| v_{K, \sigma}^{n,-} E\left[\phi_{g}\left(u_{\sigma}^{b, n}\right)\right] \\
& +k \sum_{\sigma \in \mathcal{E}^{b}}|\sigma| v_{K, \sigma}^{n,+} E\left[\phi_{d}\left(u_{\sigma}^{b, n}\right)\right]+B_{2,3} \\
= & B_{1,1}+k \sum_{\sigma \in \mathcal{E}^{b}}|\sigma| E\left[v_{K, \sigma}^{n,-} \phi_{g}\left(u_{K}^{n}\right)-v_{K, \sigma}^{n,+} \phi_{d}\left(u_{K}^{n}\right)\right]-k \sum_{\sigma \in \mathcal{E}^{b}}|\sigma| v_{K, \sigma}^{n,-} E\left[\phi_{g}\left(u_{\sigma}^{b, n}\right)\right] \\
& +k \sum_{\sigma \in \mathcal{E}^{b}}|\sigma| v_{K, \sigma}^{n,+} E\left[\phi_{d}\left(u_{\sigma}^{b, n}\right)\right]+E\left[\sum_{\sigma \in \mathcal{E}^{b}} k|\sigma|\left(v_{K, \sigma}^{n,+}-v_{K, \sigma}^{n,-}\right) \phi\left(u_{K}^{n}\right)\right] \\
= & B_{1,1}+k \sum_{\sigma \in \mathcal{E}^{b}}|\sigma| E\left[v_{K, \sigma}^{n,+}\left\{\phi\left(u_{K}^{n}\right)-\phi_{d}\left(u_{K}^{n}\right)\right\}+v_{K, \sigma}^{n,-}\left\{\phi_{g}\left(u_{K}^{n}\right)-\phi\left(u_{K}^{n}\right)\right\}\right] \\
& +k \sum_{\sigma \in \mathcal{E}^{b}}|\sigma| E\left[v_{K, \sigma}^{n,+} \phi_{d}\left(u_{\sigma}^{b, n}\right)-v_{K, \sigma}^{n,-} \phi_{g}\left(u_{\sigma}^{b, n}\right)\right] .
\end{aligned}
$$

Using the fact that $F$ is nondecreasing with respect to its first variable and nonincreasing with respect to its second one, one shows that

$$
\phi\left(u_{K}^{n}\right)-\phi_{d}\left(u_{K}^{n}\right) \geqslant 0
$$

and

$$
\phi_{g}\left(u_{K}^{n}\right)-\phi\left(u_{K}^{n}\right) \geqslant 0 .
$$


Indeed, since $\phi(a)=\int_{0}^{a} s f^{\prime}(s) \mathrm{d} s$, we have

$$
\begin{aligned}
\phi\left(u_{K}^{n}\right)-\phi_{d}\left(u_{K}^{n}\right) & =u_{K}^{n} f\left(u_{K}^{n}\right)-\int_{0}^{u_{K}^{n}} f(s) \mathrm{d} s-\left(u_{K}^{n} F\left(u_{K}^{n}, u_{K}^{n}\right)-\int_{0}^{u_{K}^{n}} F\left(u_{K}^{n}, s\right) \mathrm{d} s\right) \\
& =\int_{0}^{u_{K}^{n}}\left(F\left(u_{K}^{n}, s\right)-F(s, s)\right) \mathrm{d} s \\
& \geqslant 0
\end{aligned}
$$

and

$$
\begin{aligned}
\phi_{g}\left(u_{K}^{n}\right)-\phi\left(u_{K}^{n}\right) & =u_{K}^{n} F\left(u_{K}^{n}, u_{K}^{n}\right)-\int_{0}^{u_{K}^{n}} F\left(s, u_{K}^{n}\right) \mathrm{d} s-\left(u_{K}^{n} f\left(u_{K}^{n}\right)-\int_{0}^{u_{K}^{n}} f(s) \mathrm{d} s\right) \\
& =\int_{0}^{u_{K}^{n}} F(s, s)-F\left(s, u_{K}^{n}\right) \mathrm{d} s \\
& \geqslant 0 .
\end{aligned}
$$

Finally,

$$
B_{2,1}+B_{2,3} \geqslant B_{1,1}+k \sum_{\sigma \in \mathcal{E}^{b}}|\sigma| E\left[v_{K, \sigma}^{n,+} \phi_{d}\left(u_{\sigma}^{b, n}\right)-v_{K, \sigma}^{n,-} \phi_{g}\left(u_{\sigma}^{b, n}\right)\right]
$$

Let us now turn to an estimate of $B_{2,2}$. To do this, we use again Lemma 4.2 which gives us for all $a, b \in \mathbb{R}$ the following inequalities:

$$
\int_{a}^{b} f(t)-F(a, b) \mathrm{d} t \geqslant \int_{a}^{b} F(a, t)-F(a, a) \mathrm{d} t \geqslant \frac{1}{2 F_{2}}(f(a)-F(a, b))^{2}
$$

and

$$
\int_{a}^{b} f(t)-F(a, b) \mathrm{d} t \geqslant \int_{a}^{b} F(t, b)-F(a, b) \mathrm{d} t \geqslant \frac{1}{2 F_{1}}(f(b)-F(a, b))^{2} .
$$

Multiplying (4.14) (respectively (4.15)) by $\frac{F_{2}}{F_{1}+F_{2}}$ (respectively by $\frac{F_{1}}{F_{1}+F_{2}}$ ) and adding the two inequalities yields:

$$
\int_{a}^{b} f(t)-F(a, b) \mathrm{d} t \geqslant \frac{1}{2\left(F_{1}+F_{2}\right)}\left[(f(a)-F(a, b))^{2}+(f(b)-F(a, b))^{2}\right] .
$$

We can deduce from this last inequality that

$$
\begin{aligned}
B_{2,2}= & E\left[\sum_{(K, L) \in \mathfrak{T}_{n}} k\left|\sigma_{K, L}\right|\left\{v_{K, L}^{n,+}\left(\int_{u_{K}^{n}}^{u_{L}^{n}}\left(f(s)-F\left(u_{K}^{n}, u_{L}^{n}\right)\right) \mathrm{d} s\right)+v_{K, L}^{n,-}\left(\int_{u_{L}^{n}}^{u_{K}^{n}}\left(f(s)-F\left(u_{L}^{n}, u_{K}^{n}\right)\right) \mathrm{d} s\right)\right\}\right] \\
\geqslant & \frac{k}{2\left(F_{1}+F_{2}\right)} \sum_{(K, L) \in \mathfrak{T}_{n}}\left|\sigma_{K, L}\right| E\left[v_{K, L}^{n,+}\left\{\left(F\left(u_{K}^{n}, u_{L}^{n}\right)-f\left(u_{K}^{n}\right)\right)^{2}+\left(F\left(u_{K}^{n}, u_{L}^{n}\right)-f\left(u_{L}^{n}\right)\right)^{2}\right\}\right. \\
& \left.+v_{K, L}^{n,-}\left\{\left(f\left(u_{K}^{n}\right)-F\left(u_{L}^{n}, u_{K}^{n}\right)\right)^{2}+\left(f\left(u_{L}^{n}\right)-F\left(u_{L}^{n}, u_{K}^{n}\right)\right)^{2}\right\}\right] \\
= & B_{1,2} .
\end{aligned}
$$


In this way, combining (4.8), (4.13) and (4.16), one gets

$$
\begin{aligned}
B_{2} & =B_{2,1}+B_{2,2}+B_{2,3} \\
& \geqslant B_{1,1}+B_{1,2}+k \sum_{\sigma \in \mathcal{E}^{b}}|\sigma| E\left[v_{K, \sigma}^{n,+} \phi_{d}\left(u_{\sigma}^{b, n}\right)-v_{K, \sigma}^{n,-} \phi_{g}\left(u_{\sigma}^{b, n}\right)\right] \\
& \geqslant B_{1}+k \sum_{\sigma \in \mathcal{E}^{b}}|\sigma| E\left[v_{K, \sigma}^{n,+} \phi_{d}\left(u_{\sigma}^{b, n}\right)-v_{K, \sigma}^{n,-} \phi_{g}\left(u_{\sigma}^{b, n}\right)\right] .
\end{aligned}
$$

In summary, we showed that

$$
\begin{aligned}
\sum_{K \in \mathcal{T}} \frac{|K|}{2} E\left[\left(u_{K}^{n+1}\right)^{2}\right] & =B_{1}-B_{2}+B_{3}+\sum_{K \in \mathcal{T}} \frac{|K|}{2} E\left[\left(u_{K}^{n}\right)^{2}\right] \\
& \leqslant B_{3}+\sum_{K \in \mathcal{T}} \frac{|K|}{2} E\left[\left(u_{K}^{n}\right)^{2}\right]+k \sum_{\sigma \in \mathcal{E}^{b}}|\sigma| E\left[v_{K, \sigma}^{n,-} \phi_{g}\left(u_{\sigma}^{b, n}\right)-v_{K, \sigma}^{n,+} \phi_{d}\left(u_{\sigma}^{b, n}\right)\right] .
\end{aligned}
$$

Since for any $x \in \mathbb{R}$ we have

$$
\phi_{g}(x)=x F\left(x, u_{K}^{n}\right)-\int_{0}^{x} F\left(s, u_{K}^{n}\right) \mathrm{d} s=\int_{0}^{x}\left(F\left(x, u_{K}^{n}\right)-F\left(s, u_{K}^{n}\right)\right) \mathrm{d} s \leqslant F_{1} \int_{0}^{x}(x-s) \mathrm{d} s=F_{1} \frac{x^{2}}{2},
$$

and similarly for any $x \in \mathbb{R}, \phi_{d}(x) \geqslant-F_{2} \frac{x^{2}}{2}$, one finally gets that

$$
\begin{aligned}
\sum_{K \in \mathcal{T}} \frac{|K|}{2} E\left[\left(u_{K}^{n+1}\right)^{2}\right] & \leqslant \sum_{K \in \mathcal{T}} \frac{k|K|}{2} E\left[g\left(u_{K}^{n}\right)^{2}\right]+\sum_{K \in \mathcal{T}} \frac{|K|}{2} E\left[\left(u_{K}^{n}\right)^{2}\right]+k \sum_{\sigma \in \mathcal{E}^{b}}|\sigma| E\left[v_{K, \sigma}^{n,-} \phi_{g}\left(u_{\sigma}^{b, n}\right)-v_{K, \sigma}^{n,+} \phi_{d}\left(u_{\sigma}^{b, n}\right)\right] \\
& \leqslant \sum_{K \in \mathcal{T}} \frac{|K|}{2}\left(1+2 k C_{g}^{2}\right) E\left[\left(u_{K}^{n}\right)^{2}\right]+k \sum_{K \in \mathcal{T}}|K| g^{2}(0)+\frac{V}{2}\left(F_{1}+F_{2}\right) \sum_{\sigma \in \mathcal{E}^{b}}|\sigma| k\left(u_{\sigma}^{b, n}\right)^{2} \\
& \leqslant \sum_{K \in \mathcal{T}} \frac{|K|}{2}\left(1+2 k C_{g}^{2}\right) E\left[\left(u_{K}^{n}\right)^{2}\right]+k|D| g^{2}(0)+\frac{V}{2}\left(F_{1}+F_{2}\right) \int_{n k}^{(n+1) k} \int_{\partial D}\left(u^{b}(x, t)\right)^{2} \mathrm{~d} \gamma(x) \mathrm{d} t
\end{aligned}
$$

where we have used Jensen inequality. In this way, we deduce from the discrete Gronwall lemma that for any $n \in\{0, \ldots, N\}$

$$
\begin{aligned}
\sum_{K \in \mathcal{T}}|K| E\left[\left(u_{K}^{n}\right)^{2}\right] & \leqslant \mathrm{e}^{2 k n C_{g}^{2}}\left\{\sum_{K \in \mathcal{T}}|K| E\left[\left(u_{K}^{0}\right)^{2}\right]+2 n k|D| g^{2}(0)+V\left(F_{1}+F_{2}\right) \int_{0}^{n k} \int_{\partial D}\left(u^{b}(x, t)\right)^{2} \mathrm{~d} \gamma(x) \mathrm{d} t\right\} \\
& \leqslant \mathrm{e}^{2 T C_{g}^{2}}\left\{\left\|u_{0}\right\|_{L^{2}(D)}^{2}+2 T|D| g^{2}(0)+V\left(F_{1}+F_{2}\right) \int_{0}^{T} \int_{\partial D}\left(u^{b}(x, t)\right)^{2} \mathrm{~d} \gamma(x) \mathrm{d} t\right\} .
\end{aligned}
$$

We conclude that

$$
\left\|u_{\mathcal{T}, k}\right\|_{L^{\infty}\left(0, T ; L^{2}(\Omega \times D)\right)} \leqslant \mathrm{e}^{C_{g}^{2} T} \sqrt{\left\|u_{0}\right\|_{L^{2}(D)}^{2}+2 T|D| g^{2}(0)+V\left(F_{1}+F_{2}\right)\left\|u^{b}\right\|_{L^{2}((0, T) \times \partial D)}^{2}} .
$$

This gives the $L_{t}^{\infty} L_{\omega, x}^{2}$ stability of the approximate solution. As a consequence, we have

$$
\begin{aligned}
\left\|u_{\mathcal{T}, k}\right\|_{L^{2}(\Omega \times Q)}^{2} & =\sum_{n=0}^{N-1} \sum_{K \in \mathcal{T}} k|K| E\left[\left(u_{K}^{n}\right)^{2}\right] \\
& \leqslant T \mathrm{e}^{2 C_{g}^{2} T}\left(\left\|u_{0}\right\|_{L^{2}(D)}^{2}+2 T|D| g^{2}(0)+V\left(F_{1}+F_{2}\right)\left\|u^{b}\right\|_{L^{2}((0, T) \times \partial D)}^{2}\right) .
\end{aligned}
$$




\subsection{Weak BV estimate}

Proposition 4.3 (Weak BV estimate). Let $\mathcal{T}$ be an admissible mesh in the sense of Definition 3.1, $T>0$, $N \in \mathbb{N}^{\star}$ and let $k=\frac{T}{N} \in \mathbb{R}_{+}^{\star}$ satisfying the CFL Condition

$$
k \leqslant(1-\xi) \frac{\bar{\alpha}^{2} h}{V\left(F_{1}+F_{2}\right)},
$$

for some $\xi \in(0,1)$.

Let $\left\{u_{K}^{n}, K \in \mathcal{T}, n \in\{0, \ldots, N-1\}\right\}$ be given by the finite volume scheme (3.6).

Then the following hold:

1. There exists $C_{1} \in \mathbb{R}_{+}^{\star}$, only depending on $T,|D|, u_{0}, u^{b}, \xi, F_{1}, F_{2}, C_{g}$ and $g(0)$ such that

$$
\sum_{n=0}^{N-1} k \sum_{K \in \mathcal{T}} \sum_{\sigma \in \mathcal{E}_{K}}|\sigma| E\left[v_{K, \sigma}^{n,+}\left\{F\left(u_{K}^{n}, u_{K, \sigma}^{n}\right)-f\left(u_{K}^{n}\right)\right\}^{2}+v_{K, \sigma}^{n,-}\left\{F\left(u_{K, \sigma}^{n}, u_{K}^{n}\right)-f\left(u_{K}^{n}\right)\right\}^{2}\right] \leqslant C_{1} .
$$

2. There exists $C_{2} \in \mathbb{R}_{+}^{\star}$, only depending on $T,|D|, \bar{\alpha}, u_{0}, u^{b}, \xi, F_{1}, F_{2}, C_{g}$ and $g(0)$ such that

$$
\begin{aligned}
\sum_{n=0}^{N-1} k \sum_{(K, L) \in \mathfrak{T}_{n}}\left|\sigma_{K, L}\right| E[ & v_{K, L}^{n,+}\left\{\max _{u_{L}^{n} \leqslant c \leqslant d \leqslant u_{K}^{n}}(F(d, c)-f(d))+\max _{u_{L}^{n} \leqslant c \leqslant d \leqslant u_{K}^{n}}(F(d, c)-f(c))\right\} \\
& \left.+v_{K, L}^{n,-}\left\{\max _{u_{L}^{n} \leqslant c \leqslant d \leqslant u_{K}^{n}}(f(d)-F(c, d))+\max _{u_{L}^{n} \leqslant c \leqslant d \leqslant u_{K}^{n}}(f(c)-F(c, d))\right\}\right] \leqslant C_{2} h^{-1 / 2},
\end{aligned}
$$

where

$$
\mathfrak{T}_{n}=\left\{(K, L) \in \mathcal{T}^{2}: L \in \mathcal{N}(K) \text { and } u_{K}^{n}>u_{L}^{n}\right\} .
$$

Proof. Recall that by multiplying the finite volume scheme (3.6) by $k u_{K}^{n}$, taking the expectation and summing over $K \in \mathcal{T}$ yields equality (4.3) and after summing over $n \in\{0, \ldots, N\}$, we have:

$$
\sum_{n=0}^{N-1} \sum_{K \in \mathcal{T}} \frac{|K|}{2} E\left[\left(u_{K}^{n+1}\right)^{2}-\left(u_{K}^{n}\right)^{2}\right]=\sum_{n=0}^{N-1}\left(B_{1}-B_{2}+B_{3}\right),
$$

where

$$
\begin{aligned}
& B_{1}=\sum_{K \in \mathcal{T}} \frac{k^{2}}{2|K|} E\left[\left(\sum_{\sigma \in \mathcal{E}_{K}}|\sigma|\left\{v_{K, \sigma}^{n,+}\left(F\left(u_{K}^{n}, u_{K, \sigma}^{n}\right)-f\left(u_{K}^{n}\right)\right)-v_{K, \sigma}^{n,-}\left(F\left(u_{K, \sigma}^{n}, u_{K}^{n}\right)-f\left(u_{K}^{n}\right)\right)\right\}\right)^{2}\right] \\
& B_{2}=\sum_{K \in \mathcal{T}} k E\left[\sum_{\sigma \in \mathcal{E}_{K}}|\sigma|\left\{v_{K, \sigma}^{n,+}\left(F\left(u_{K}^{n}, u_{K, \sigma}^{n}\right)-f\left(u_{K}^{n}\right)\right)-v_{K, \sigma}^{n,-}\left(F\left(u_{K, \sigma}^{n}, u_{K}^{n}\right)-f\left(u_{K}^{n}\right)\right)\right\} u_{K}^{n}\right]
\end{aligned}
$$

and

$$
B_{3}=\sum_{K \in \mathcal{T}} \frac{k|K|}{2} E\left[\left(g\left(u_{K}^{n}\right)\right)^{2}\right] .
$$

- Study of $B_{1}$ : Similarly to (4.5), it follows from the CFL Condition (4.17) and the mesh properties (3.2) that

$$
\frac{k}{|K|} \sum_{\sigma \in \mathcal{E}_{K}}|\sigma|\left(v_{K, \sigma}^{n,+}+v_{K, \sigma}^{n,-}\right) \leqslant k V \frac{|\partial K|}{|K|} \leqslant \frac{1-\xi}{F_{1}+F_{2}} .
$$


Then, by using Cauchy-Schwarz inequality and Jensen inequality, we get similarly to (4.6) the following estimate

$$
B_{1} \leqslant \frac{k(1-\xi)}{2\left(F_{1}+F_{2}\right)} \sum_{K \in \mathcal{T}} \sum_{\sigma \in \mathcal{E}_{K}}|\sigma| E\left[v_{K, \sigma}^{n,+}\left(F\left(u_{K}^{n}, u_{K, \sigma}^{n}\right)-f\left(u_{K}^{n}\right)\right)^{2}+v_{K, \sigma}^{n,-}\left(F\left(u_{K, \sigma}^{n}, u_{K}^{n}\right)-f\left(u_{K}^{n}\right)\right)^{2}\right] .
$$

By denoting again

$$
\mathfrak{T}_{n}=\left\{(K, L) \in \mathcal{T}^{2}: L \in \mathcal{N}(K) \text { and } u_{K}^{n}>u_{L}^{n}\right\}
$$

and by reordering the summation in the right hand side of (4.19) thanks to the formula (4.7), one gets:

$$
B_{1} \leqslant B_{1,1}+B_{1,2}
$$

where

$$
\begin{aligned}
B_{1,1}= & \frac{k(1-\xi)}{2\left(F_{1}+F_{2}\right)} \sum_{\sigma \in \mathcal{E}^{b}}|\sigma| E\left[v_{K, \sigma}^{n,+}\left(F\left(u_{K}^{n}, u_{\sigma}^{b, n}\right)-f\left(u_{K}^{n}\right)\right)^{2}+v_{K, \sigma}^{n,-}\left(F\left(u_{\sigma}^{b, n}, u_{K}^{n}\right)-f\left(u_{K}^{n}\right)\right)^{2}\right] \\
B_{1,2}= & \frac{k(1-\xi)}{2\left(F_{1}+F_{2}\right)} \sum_{(K, L) \in \mathcal{T}_{n}}\left|\sigma_{K, L}\right| E\left[v_{K, L}^{n,+}\left\{\max _{u_{L}^{n} \leqslant c \leqslant d \leqslant u_{K}^{n}}(F(d, c)-f(d))^{2}+\max _{u_{L}^{n} \leqslant c \leqslant d \leqslant u_{K}^{n}}(F(d, c)-f(c))^{2}\right\}\right. \\
& \left.+v_{K, L}^{n,-}\left\{\max _{u_{L}^{n} \leqslant c \leqslant d \leqslant u_{K}^{n}}(f(d)-F(c, d))^{2}+\max _{u_{L}^{n} \leqslant c \leqslant d \leqslant u_{K}^{n}}(f(c)-F(c, d))^{2}\right\}\right] .
\end{aligned}
$$

- Study of $B_{2}$ : By reordering the summation and using again the notation $\phi(a)=\int_{0}^{a} s f^{\prime}(s) \mathrm{d} s, B_{2}$ can be decomposed, as in the proof of Proposition 4.1, in the following way

$$
B_{2}=B_{2,1}+B_{2,2}+B_{2,3}
$$

where

$$
\begin{aligned}
& B_{2,1}=k \sum_{\sigma \in \mathcal{E}^{b}}|\sigma| E\left[u_{K}^{n}\left\{v_{K, \sigma}^{n,+}\left(F\left(u_{K}^{n}, u_{\sigma}^{b, n}\right)-f\left(u_{K}^{n}\right)\right)-v_{K, \sigma}^{n,-}\left(F\left(u_{\sigma}^{b, n}, u_{K}^{n}\right)-f\left(u_{K}^{n}\right)\right)\right\}\right], \\
& B_{2,2}=E\left[\sum_{(K, L) \in \mathfrak{T}_{n}} k\left|\sigma_{K, L}\right|\left\{v_{K, L}^{n,+}\left(\int_{u_{K}^{n}}^{u_{L}^{n}}\left(f(s)-F\left(u_{K}^{n}, u_{L}^{n}\right)\right) \mathrm{d} s\right)+v_{K, L}^{n,-}\left(\int_{u_{L}^{n}}^{u_{K}^{n}}\left(f(s)-F\left(u_{L}^{n}, u_{K}^{n}\right)\right) \mathrm{d} s\right)\right\}\right],
\end{aligned}
$$

and

$$
B_{2,3}=E\left[\sum_{\sigma \in \mathcal{E}^{b}} k|\sigma|\left(v_{K, \sigma}^{n,+}-v_{K, \sigma}^{n,-}\right) \phi\left(u_{K}^{n}\right)\right] .
$$

Following the proof of Proposition 4.1 one shows that

$$
\begin{aligned}
B_{2,1} \geqslant & \frac{k}{2\left(F_{1}+F_{2}\right)} \sum_{\sigma \in \mathcal{E}^{b}}|\sigma| E\left[v_{K, \sigma}^{n,+}\left(F\left(u_{K}^{n}, u_{\sigma}^{b, n}\right)-f\left(u_{K}^{n}\right)\right)^{2}+v_{K, \sigma}^{n,-}\left(F\left(u_{\sigma}^{b, n}, u_{K}^{n}\right)-f\left(u_{K}^{n}\right)\right)^{2}\right] \\
& +k \sum_{\sigma \in \mathcal{E}^{b}}|\sigma| E\left[v_{K, \sigma}^{n,+}\left\{\phi_{d}\left(u_{\sigma}^{b, n}\right)-\phi_{d}\left(u_{K}^{n}\right)\right\}+v_{K, \sigma}^{n,-}\left\{\phi_{g}\left(u_{K}^{n}\right)-\phi_{g}\left(u_{\sigma}^{b, n}\right)\right\}\right] \\
= & \frac{1}{1-\xi} B_{1,1}+k \sum_{\sigma \in \mathcal{E}^{b}}|\sigma| E\left[v_{K, \sigma}^{n,+}\left\{\phi_{d}\left(u_{\sigma}^{b, n}\right)-\phi_{d}\left(u_{K}^{n}\right)\right\}+v_{K, \sigma}^{n,-}\left\{\phi_{g}\left(u_{K}^{n}\right)-\phi_{g}\left(u_{\sigma}^{b, n}\right)\right\}\right] \\
\geqslant & \frac{1}{1-\xi} B_{1,1}+k \sum_{\sigma \in \mathcal{E}^{b}}|\sigma| E\left[v_{K, \sigma}^{n,-} \phi_{g}\left(u_{K}^{n}\right)-v_{K, \sigma}^{n,+} \phi_{d}\left(u_{K}^{n}\right)\right] \\
& +k \sum_{\sigma \in \mathcal{E}^{b}}|\sigma| E\left[v_{K, \sigma}^{n,+} \phi_{d}\left(u_{\sigma}^{b, n}\right)-v_{K, \sigma}^{n,-} \phi_{g}\left(u_{\sigma}^{b, n}\right)\right] .
\end{aligned}
$$


We still follow the proof of Proposition 4.1. In particular we use the fact that $F$ is nondecreasing with respect to its first variable and nonincreasing with respect to its second variable, and we deduce that

$$
\begin{aligned}
B_{2,1}+B_{2,3} \geqslant & \frac{1}{1-\xi} B_{1,1}+k \sum_{\sigma \in \mathcal{E}^{b}}|\sigma| E\left[v_{K, \sigma}^{n,-} \phi_{g}\left(u_{K}^{n}\right)-v_{K, \sigma}^{n,+} \phi_{d}\left(u_{K}^{n}\right)\right]+B_{2,3} \\
& +k \sum_{\sigma \in \mathcal{E}^{b}}|\sigma| E\left[v_{K, \sigma}^{n,+} \phi_{d}\left(u_{\sigma}^{b, n}\right)-v_{K, \sigma}^{n,-} \phi_{g}\left(u_{\sigma}^{b, n}\right)\right] \\
= & \frac{1}{1-\xi} B_{1,1}+k \sum_{\sigma \in \mathcal{E}^{b}}|\sigma| E\left[v_{K, \sigma}^{n,-} \phi_{g}\left(u_{K}^{n}\right)-v_{K, \sigma}^{n,+} \phi_{d}\left(u_{K}^{n}\right)\right]+E\left[\sum_{\sigma \in \mathcal{E}^{b}} k|\sigma|\left(v_{K, \sigma}^{n,+}-v_{K, \sigma}^{n,-}\right) \phi\left(u_{K}^{n}\right)\right] \\
& +k \sum_{\sigma \in \mathcal{E}^{b}}|\sigma| E\left[v_{K, \sigma}^{n,+} \phi_{d}\left(u_{\sigma}^{b, n}\right)-v_{K, \sigma}^{n,-} \phi_{g}\left(u_{\sigma}^{b, n}\right)\right] \\
\geqslant & \frac{1}{1-\xi} B_{1,1}+k \sum_{\sigma \in \mathcal{E}^{b}}|\sigma| E\left[v_{K, \sigma}^{n,+} \phi_{d}\left(u_{\sigma}^{b, n}\right)-v_{K, \sigma}^{n,-} \phi_{g}\left(u_{\sigma}^{b, n}\right)\right] .
\end{aligned}
$$

Let us now turn to an estimate of $B_{2,2}$. For this purpose, let $a, b \in \mathbb{R}$ and define

$$
\mathcal{C}(a, b)=\left\{(c, d) \in[\min (a, b), \max (a, b)]^{2}:(d-c)(b-a) \geqslant 0\right\} .
$$

Thanks to the monotonicity of $F$, the following inequality holds for any $(c, d) \in \mathcal{C}(a, b)$ :

$$
\int_{a}^{b} f(s)-F(a, b) \mathrm{d} s \geqslant \int_{c}^{d} f(s)-F(a, b) \mathrm{d} s \geqslant \int_{c}^{d} f(s)-F(c, d) \mathrm{d} s .
$$

We now use again Lemma 4.2 and deduce that for all $(c, d) \in \mathcal{C}(a, b)$ :

$$
\int_{a}^{b} f(s)-F(a, b) \mathrm{d} s \geqslant \int_{c}^{d} f(s)-F(c, d) \mathrm{d} s \geqslant \int_{c}^{d} F(c, s)-F(c, d) \mathrm{d} s \geqslant \frac{1}{2 F_{2}}(f(c)-F(c, d))^{2}
$$

and

$$
\int_{a}^{b} f(s)-F(a, b) \mathrm{d} s \geqslant \int_{c}^{d} f(s)-F(c, d) \mathrm{d} s \geqslant \int_{c}^{d} F(s, d)-F(c, d) \mathrm{d} s \geqslant \frac{1}{2 F_{1}}(f(d)-F(c, d))^{2} .
$$

Multiplying (4.22) (respectively (4.23)) by $\frac{F_{2}}{F_{1}+F_{2}}$ (respectively by $\frac{F_{1}}{F_{1}+F_{2}}$ ), taking the maximum for $(c, d) \in \mathcal{C}(a, b)$ and adding the two inequalities yields:

$$
\int_{a}^{b} f(s)-F(a, b) \mathrm{d} s \geqslant \frac{1}{2\left(F_{1}+F_{2}\right)}\left[\max _{(c, d) \in \mathcal{C}(a, b)}(f(c)-F(c, d))^{2}+\max _{(c, d) \in \mathcal{C}(a, b)}(f(d)-F(c, d))^{2}\right] .
$$

We can deduce from this last inequality that

$$
\begin{aligned}
B_{2,2} \geqslant & k \sum_{(K, L) \in \mathfrak{T}_{n}} \frac{\left|\sigma_{K, L}\right|}{2\left(F_{1}+F_{2}\right)} E\left[v_{K, L}^{n,+}\left\{\max _{u_{L}^{n} \leqslant c \leqslant d \leqslant u_{K}^{n}}(F(d, c)-f(d))^{2}+\max _{u_{L}^{n} \leqslant c \leqslant d \leqslant u_{K}^{n}}(F(d, c)-f(c))^{2}\right\}\right. \\
& \left.+v_{K, L}^{n,-}\left\{\max _{u_{L}^{n} \leqslant c \leqslant d \leqslant u_{K}^{n}}(f(d)-F(c, d))^{2}+\max _{u_{L}^{n} \leqslant c \leqslant d \leqslant u_{K}^{n}}(f(c)-F(c, d))^{2}\right\}\right] . \\
= & \frac{1}{1-\xi} B_{1,2} .
\end{aligned}
$$


In this way, using (4.21) and (4.24), one gets

$$
\begin{aligned}
B_{2} & =B_{2,1}+B_{2,2}+B_{2,3} \\
& \geqslant \frac{1}{1-\xi}\left(B_{1,1}+B_{1,2}\right)+k \sum_{\sigma \in \mathcal{E}^{b}}|\sigma| E\left[v_{K, \sigma}^{n,+} \phi_{d}\left(u_{\sigma}^{b, n}\right)-v_{K, \sigma}^{n,-} \phi_{g}\left(u_{\sigma}^{b, n}\right)\right] .
\end{aligned}
$$

- Study of $B_{3}$ : Using the constant $C_{\text {est }}$ introduced in the stability result stated in Proposition 4.1,

$$
\begin{aligned}
\sum_{n=0}^{N-1} B_{3} & =\sum_{n=0}^{N-1} \sum_{K \in \mathcal{T}} \frac{k|K|}{2} E\left[\left(g\left(u_{K}^{n}\right)\right)^{2}\right] \\
& \leqslant T|D| g^{2}(0)+C_{g}^{2} \sum_{n=0}^{N-1} k \sum_{K \in \mathcal{T}}|K| E\left[\left(u_{K}^{n}\right)^{2}\right] \\
& \leqslant T|D| g^{2}(0)+C_{g}^{2} T C_{\text {est }}^{2} .
\end{aligned}
$$

Finally, since

$$
\begin{aligned}
\sum_{n=0}^{N-1} \sum_{K \in \mathcal{T}} \frac{|K|}{2} E\left[\left(u_{K}^{n+1}\right)^{2}-\left(u_{K}^{n}\right)^{2}\right] & =\sum_{K \in \mathcal{T}} \frac{|K|}{2} E\left[\left(u_{K}^{N}\right)^{2}-\left(u_{K}^{0}\right)^{2}\right] \\
& =\sum_{n=0}^{N-1}\left(B_{1}-B_{2}+B_{3}\right)
\end{aligned}
$$

one gets with (4.20), (4.25), and (4.26)

$$
\begin{aligned}
0 \leqslant \sum_{K \in \mathcal{T}} \frac{|K|}{2} E\left[\left(u_{K}^{N}\right)^{2}\right] \leqslant & \frac{1}{2}|| u_{0} \|_{L^{2}(D)}^{2}+k \sum_{\sigma \in \mathcal{E}^{b}}|\sigma| E\left[v_{K, \sigma}^{n,-} \phi_{g}\left(u_{\sigma}^{b, n}\right)-v_{K, \sigma}^{n,+} \phi_{d}\left(u_{\sigma}^{b, n}\right)\right] \\
& +\left(1-\frac{1}{1-\xi}\right) \sum_{n=0}^{N-1}\left(B_{1,1}+B_{1,2}\right)+T|D| g^{2}(0)+C_{g}^{2} T C_{\text {est }}^{2} .
\end{aligned}
$$

Then, following again the proof of Proposition 4.1, and using in particular the fact that for any $x \in \mathbb{R}$,

$$
\phi_{g}(x) \leqslant F_{1} \frac{x^{2}}{2} \text { and } \phi_{d}(x) \geqslant-F_{2} \frac{x^{2}}{2},
$$

we get

$$
\begin{gathered}
\frac{\xi}{2\left(F_{1}+F_{2}\right)} \sum_{n=0}^{N-1} k \sum_{\sigma \in \mathcal{E}^{b}}|\sigma| E\left[v_{K, \sigma}^{n,+}\left(F\left(u_{K}^{n}, u_{\sigma}^{b, n}\right)-f\left(u_{K}^{n}\right)\right)^{2}+v_{K, \sigma}^{n,-}\left(F\left(u_{\sigma}^{b, n}, u_{K}^{n}\right)-f\left(u_{K}^{n}\right)\right)^{2}\right] \\
+\frac{\xi}{2\left(F_{1}+F_{2}\right)} \sum_{n=0}^{N-1} k \sum_{(K, L) \in \mathfrak{T}_{n}}\left|\sigma_{K, L}\right| E\left[v_{K, L}^{n,+}\left\{\max _{u_{L}^{n} \leqslant c \leqslant d \leqslant u_{K}^{n}}(F(d, c)-f(d))^{2}+\max _{u_{L}^{n} \leqslant s \leqslant d \leqslant u_{K}^{n}}(F(d, c)-f(c))^{2}\right\}\right. \\
\left.+v_{K, L}^{n,-}\left\{\max _{u_{L}^{n} \leqslant c \leqslant d \leqslant u_{K}^{n}}(f(d)-F(c, d))^{2}+\max _{u_{L}^{n} \leqslant c \leqslant d \leqslant u_{K}^{n}}(f(c)-F(c, d))^{2}\right\}\right] \\
\leqslant \frac{1}{2}\left\|u_{0}\right\|_{L^{2}(D)}^{2}+V \frac{\left(F_{1}+F_{2}\right)}{2}\left\|u^{b}\right\|_{L^{2}((0, T) \times \partial D)}^{2}+T|D| g^{2}(0)+C_{g}^{2} T C_{e s t}^{2}
\end{gathered}
$$


which, in turn, gives the existence of $C_{1} \in \mathbb{R}_{+}^{*}$, only depending on $T,|D|, V, C_{g}, g(0), F_{1}, F_{2}, \xi,\left\|u_{0}\right\|_{L^{2}(D)}$ and $\left\|u^{b}\right\|_{L^{2}((0, T) \times \partial D)}^{2}$ such that

$$
\begin{aligned}
& \sum_{n=0}^{N-1} k \sum_{\sigma \in \mathcal{E}^{b}}|\sigma| E\left[v_{K, \sigma}^{n,+}\left(F\left(u_{K}^{n}, u_{\sigma}^{b, n}\right)-f\left(u_{K}^{n}\right)\right)^{2}+v_{K, \sigma}^{n,-}\left(F\left(u_{\sigma}^{b, n}, u_{K}^{n}\right)-f\left(u_{K}^{n}\right)\right)^{2}\right] \\
& +\sum_{n=0}^{N-1} k \sum_{(K, L) \in \mathcal{T}_{n}}\left|\sigma_{K, L}\right| E\left[v_{K, \sigma}^{n,+}\left\{\max _{u_{L}^{n} \leqslant c \leqslant d \leqslant u_{K}^{n}}(F(d, c)-f(d))^{2}+\max _{u_{L}^{n} \leqslant c \leqslant d \leqslant u_{K}^{n}}(F(d, c)-f(c))^{2}\right\}\right. \\
& \left.+v_{K, \sigma}^{n,-}\left\{\max _{u_{L}^{n} \leqslant c \leqslant d \leqslant u_{K}^{n}}(f(d)-F(c, d))^{2}+\max _{u_{L}^{n} \leqslant c \leqslant d \leqslant u_{K}^{n}}(f(c)-F(c, d))^{2}\right\}\right] \leqslant C_{1} .
\end{aligned}
$$

Moreover by reordering the summation and taking $c=u_{L}^{n}$ and $d=u_{K}^{n}$ in the maximum, we have in particular

$$
\sum_{n=0}^{N-1} k \sum_{K \in \mathcal{T}} \sum_{\sigma \in \mathcal{E}_{K}}|\sigma| E\left[v_{K, \sigma}^{n,+}\left\{F\left(u_{K}^{n}, u_{K, \sigma}^{n}\right)-f\left(u_{K}^{n}\right)\right\}^{2}+v_{K, \sigma}^{n,-}\left\{F\left(u_{K, \sigma}^{n}, u_{K}^{n}\right)-f\left(u_{K}^{n}\right)\right\}^{2}\right] \leqslant C_{1},
$$

which proves the first point of the proposition.

Let us now turn to the second point of the proposition. To do this, we aim to estimate

$$
\begin{aligned}
\left\{\sum _ { n = 0 } ^ { N - 1 } k \sum _ { ( K , L ) \in \mathfrak { T } _ { n } } | \sigma _ { K , L } | E \left[v_{K, L}^{n,+}\left\{\max _{u_{L}^{n} \leqslant c \leqslant d \leqslant u_{K}^{n}}(F(d, c)-f(d))+\max _{u_{L}^{n} \leqslant c \leqslant d \leqslant u_{K}^{n}}(F(d, c)-f(c))\right\}\right.\right. & \\
& \left.\left.+v_{K, L}^{n,-}\left\{\max _{u_{L}^{n} \leqslant c \leqslant d \leqslant u_{K}^{n}}(f(d)-F(c, d))+\max _{u_{L}^{n} \leqslant c \leqslant d \leqslant u_{K}^{n}}(f(c)-F(c, d))\right\}\right]\right\}
\end{aligned}
$$

Let us denote by

$$
T_{1}=\max _{u_{L}^{n} \leqslant c \leqslant d \leqslant u_{K}^{n}}(F(d, c)-f(d))+\max _{u_{L}^{n} \leqslant c \leqslant d \leqslant u_{K}^{n}}(F(d, c)-f(c))
$$

and

$$
T_{2}=\max _{u_{L}^{n} \leqslant c \leqslant d \leqslant u_{K}^{n}}(f(d)-F(c, d))+\max _{u_{L}^{n} \leqslant c \leqslant d \leqslant u_{K}^{n}}(f(c)-F(c, d)) .
$$

Using Cauchy-Schwarz inequality, one gets

$$
\begin{aligned}
& \left(\sum_{n=0}^{N-1} k \sum_{(K, L) \in \mathfrak{T}_{n}}\left|\sigma_{K, L}\right| E\left[v_{K, L}^{n,+} T_{1}+v_{K, L}^{n,-} T_{2}\right]\right)^{2} \\
& \quad \leqslant\left(\sum_{n=0}^{N-1} k \sum_{(K, L) \in \mathfrak{T}_{n}}\left|\sigma_{K, L}\right|\left(v_{K, L}^{n,+}+v_{K, L}^{n,-}\right)\right) \times\left(\sum_{n=0}^{N-1} k \sum_{(K, L) \in \mathfrak{T}_{n}}\left|\sigma_{K, L}\right| E\left[\frac{\left(v_{K, L}^{n,+} T_{1}+v_{K, L}^{n,-} T_{2}\right)^{2}}{v_{K, L}^{n,+}+v_{K, L}^{n,-}}\right]\right) \\
& \quad \leqslant\left(\sum_{n=0}^{N-1} k \sum_{(K, L) \in \mathfrak{T}_{n}}\left|\sigma_{K, L}\right|\left(v_{K, L}^{n,+}+v_{K, L}^{n,-}\right)\right) \times\left(\sum_{n=0}^{N-1} k \sum_{(K, L) \in \mathfrak{T}_{n}}\left|\sigma_{K, L}\right| E\left[v_{K, L}^{n,+} T_{1}^{2}+v_{K, L}^{n,-} T_{2}^{2}\right]\right),
\end{aligned}
$$

where we have used the convexity inequality

$$
\left(\frac{v_{K, L}^{n,+} T_{1}+v_{K, L}^{n,-} T_{2}}{v_{K, L}^{n,+}+v_{K, L}^{n,-}}\right)^{2} \leq \frac{v_{K, L}^{n,+}}{v_{K, L}^{n,+}+v_{K, L}^{n,-}} T_{1}^{2}+\frac{v_{K, L}^{n,-}}{v_{K, L}^{n,+}+v_{K, L}^{n,-}} T_{2}^{2}
$$


We have then, by using the consequence of the mesh properties (3.2)

$$
\sum_{n=0}^{N-1} k \sum_{(K, L) \in \mathfrak{T}_{n}}\left|\sigma_{K, L}\right|\left(v_{K, L}^{n,+}+v_{K, L}^{n,-}\right) \leqslant T V \sum_{K \in \mathcal{T}}|\partial K| \leqslant T V \frac{1}{\bar{\alpha}^{2} h} \sum_{K \in \mathcal{T}}|K| \leqslant T V \frac{1}{\bar{\alpha}^{2} h}|D| .
$$

Finally, using (4.27) and (4.28), the fact that

$$
\begin{aligned}
& T_{1}^{2} \leqslant 2\left\{\max _{u_{L}^{n} \leqslant c \leqslant d \leqslant u_{K}^{n}}(F(d, c)-f(d))^{2}+\max _{u_{L}^{n} \leqslant c \leqslant d \leqslant u_{K}^{n}}(F(d, c)-f(c))^{2}\right\} \\
& T_{2}^{2} \leqslant 2\left\{\max _{u_{L}^{n} \leqslant c \leqslant d \leqslant u_{K}^{n}}(f(d)-F(c, d))^{2}+\max _{u_{L}^{n} \leqslant c \leqslant d \leqslant u_{K}^{n}}(f(c)-F(c, d))^{2}\right\}
\end{aligned}
$$

one finally gets

$$
\begin{aligned}
\left(\sum_{n=0}^{N-1} k \sum_{(K, L) \in \mathfrak{T}_{n}}\left|\sigma_{K, L}\right| E\right. & {\left[v_{K, L}^{n}\left\{\max _{u_{L}^{n} \leqslant c \leqslant d \leqslant u_{K}^{n}}(F(d, c)-f(d))+\max _{u_{L}^{n} \leqslant c \leqslant d \leqslant u_{K}^{n}}(F(d, c)-f(c))\right\}\right.} \\
& \left.\left.+v_{L, K}^{n}\left\{\max _{u_{L}^{n} \leqslant c \leqslant d \leqslant u_{K}^{n}}(f(d)-F(c, d))+\max _{u_{L}^{n} \leqslant c \leqslant d \leqslant u_{K}^{n}}(f(c)-F(c, d))\right\}\right]\right)^{2} \leqslant \frac{2 T V C_{1}|D|}{\bar{\alpha}^{2} h},
\end{aligned}
$$

which concludes the proof of the second point of the proposition.

\subsection{Convergence of the finite volume approximate solution}

First of all, note that the estimates stated in Proposition 4.1 only provide (up to a subsequence) weak convergences for $u_{\mathcal{T}, k}$. Moreover, due to the nonlinearity of $f$ and $g$, one needs compactness arguments to pass to the limit in the nonlinear terms and these arguments have to be compatible with the random variable. The concept of Young measures is appropriate here and the technique is based on the notion of narrow convergence of Young measures (or entropy processes), we refer to Balder [1] but also to Eymard-Gallouët-Herbin [12].

In this way, taking a sequence of approximate finite volume solution $u_{\mathcal{T}, k}$, it converges (up to a subsequence still denoted $u_{\mathcal{T}, k}$ ) in the sense of Young measures to an "entropy process" denoted by $\mathbf{u}$ which belongs to $L^{2}(\Omega \times Q \times(0,1))$. Precisely, given a Carathéodory function $\Psi: \Omega \times Q \times \mathbb{R} \rightarrow \mathbb{R}$ such that $\Psi\left(., u_{\mathcal{T}, k}\right)$ is uniformly integrable, one has:

$$
E\left[\int_{Q} \Psi\left(., u_{\mathcal{T}, k}\right) \mathrm{d} x \mathrm{~d} t\right] \rightarrow E\left[\int_{Q} \int_{0}^{1} \Psi(., \mathbf{u}(., \alpha)) \mathrm{d} \alpha \mathrm{d} x \mathrm{~d} t\right] .
$$

A proof of this result can be found in [3], Section A.3.2. We recall that a function $\Psi: \Omega \times Q \times \mathbb{R} \rightarrow \mathbb{R}$ is a Carathéodory function if for almost any $(\omega, x, t) \in \Omega \times Q$ the function $\nu \mapsto \Psi(\omega, x, t, \nu)$ is continuous and for all $\nu \in \mathbb{R}$, the function $(\omega, x, t) \mapsto \Psi(\omega, x, t, \nu)$ is measurable. We also recall that a sequence $\left(\psi_{n}\right)_{n \geq 0}$ of functions $\psi_{n}: \Omega \times Q \rightarrow \mathbb{R}$ is said to be uniformly integrable on the domain $\Omega \times Q$ if it satisfies the following properties:

- $\left(\psi_{n}\right)_{n \geq 0}$ is bounded in $L^{1}(\Omega \times Q)$.

- $\left(\psi_{n}\right)_{n \geq 0}$ is equi-integrable, that is to say that for any $\varepsilon>0$, there exists $\delta>0$ such that for any measurable set $A$ of $\Omega \times Q$ satisfying $\left(\mathcal{L}^{d+1} \otimes P\right)(A) \leqslant \delta$, we have for any $n \in \mathbb{N}$,

$$
\int_{A}\left|\psi_{n}(\omega, x, t)\right| \mathrm{d} x \mathrm{~d} t \mathrm{~d} P \leqslant \varepsilon
$$

(where $\mathcal{L}^{d+1}$ is the $d+1$-dimensional Lebesgue measure). 
Remark 4.4 (On the measurability of $\mathbf{u}$ ). Since $u_{\mathcal{T}, k}$ is bounded in the Hilbert space $\mathcal{N}_{w}^{2}\left(0, T, L^{2}(D)\right.$ ), by identification one shows that $u_{\mathcal{T}, k} \rightarrow \int_{0}^{1} \mathbf{u}(., \alpha) \mathrm{d} \alpha$ weakly in $L^{2}(\Omega \times Q)$ so that $\int_{0}^{1} \mathbf{u}(., \alpha) \mathrm{d} \alpha$ is a predictable process with values in $L^{2}(D)$. An interesting point is the measurability of $\mathbf{u}$ with respect to all its variables $(\omega, x, t, \alpha)$. Revisiting the work of Panov [20] with the $\sigma$-field $\mathcal{P}_{T} \otimes \mathcal{L}(D)$, one shows that $\mathbf{u}$ is measurable for the $\sigma$-field $\mathcal{P}_{T} \otimes \mathcal{L}(D \times] 0,1[)$, thus $\mathbf{u} \in \mathcal{N}_{w}^{2}\left(0, T, L^{2}(D \times] 0,1[)\right)$ (see Appendix A.3.3, p. $707[3]$ ).

Remark 4.5 $\left(L^{\infty}\left(0, T ; L^{2}(\Omega \times D \times(0,1))\right)\right)$ regularity of $\left.\mathbf{u}\right)$. Since the sequence of approximate solutions $u_{\mathcal{T}, k}$ is bounded in $L^{\infty}\left(0, T ; L^{2}(\Omega \times D)\right)$ according to Proposition 4.1, following [3] (Rem. 2.4, pp. 667-668) we show that $\mathbf{u} \in L^{\infty}\left(0, T ; L^{2}(\Omega \times D \times(0,1))\right)$.

Note that if one is able to show that $\mathbf{u}$ is a measure-valued entropy solution of problem (1.1) in the sense of Definition 2.2, then, using Theorem 2.3, we will be able to conclude that all the sequence $u_{\mathcal{T}, k}$ converges in $L^{1}(\Omega \times Q)$ to the unique stochastic entropy solution of (1.1) in the sense of Definition 2.1. Since $\mathbf{u}$ satisfied the regularities required by Definition 2.2, it remains to show that $\mathbf{u}$ satisfies the following entropy inequalities:

$\forall \eta \in \mathcal{A}, \forall \varphi \in \mathcal{D}^{+}\left(\mathbb{R}^{d} \times[0, T)\right)$ and P-a.s. in $\Omega$

$$
\begin{aligned}
0 \leqslant & \int_{D} \eta\left(u_{0}\right) \varphi(x, 0) \mathrm{d} x+\int_{Q} \int_{0}^{1}\left\{\eta(\mathbf{u}(., \alpha)) \partial_{t} \varphi(x, t)+\Phi(\mathbf{u}(., \alpha)) \boldsymbol{v}(x, t) . \nabla_{x} \varphi(x, t)\right\} \mathrm{d} \alpha \mathrm{d} x \mathrm{~d} t \\
& +\int_{0}^{T} \int_{D} \int_{0}^{1} \eta^{\prime}(\mathbf{u}(., \alpha)) g(\mathbf{u}(., \alpha)) \varphi(x, t) \mathrm{d} \alpha \mathrm{d} x \mathrm{~d} W(t)+C_{f} V \int_{0}^{T} \int_{\partial D} \varphi(x, t) \eta\left(u^{b}(x, t)\right) \mathrm{d} \gamma(t) \mathrm{d} t \\
& +\frac{1}{2} \int_{Q} \int_{0}^{1} g^{2}(\mathbf{u}(., \alpha)) \eta^{\prime \prime}(\mathbf{u}(., \alpha)) \varphi(x, t) \mathrm{d} \alpha \mathrm{d} x \mathrm{~d} t .
\end{aligned}
$$

This is the aim of the next section.

\section{Convergence OF THE SCHEME}

In order to show the convergence of the finite volume scheme (3.6), we are going to use the following lemma as in [5], which states that any general monotone numerical flux can be split into the sum of a Godunov flux and a modified Lax-Friedrichs flux (also known as Rusanov flux). More precisely, we have the following result, whose proof can be found in [8] or in [5].

Lemma 5.1. Any monotone flux F (i.e. any numerical flux satisfying Def. 3.3) can be written as a convex combination of a Godunov flux and a modified Lax-Friedrichs flux as follows:

For any $a, b \in \mathbb{R}$ there exists $\theta(a, b) \in[0,1]$ such that

$$
F(a, b)=\theta(a, b) F^{G}(a, b)+(1-\theta(a, b)) F^{L F}(a, b),
$$

where $F^{G}$ is a Godunov flux, that is to say a flux defined by

$$
F^{G}(a, b)= \begin{cases}\min _{s \in[a, b]} f(s) & \text { if } a \leqslant b \\ \max _{s \in[b, a]} f(s) & \text { if } a \geqslant b\end{cases}
$$

and $F^{L F}$ is a modified Lax-Friedrichs flux with parameter $M_{F}=\max \left(F_{1}, F_{2}\right)$ satisfying:

$$
F^{L F}(a, b)=\frac{f(a)+f(b)}{2}-M_{F}(b-a) .
$$


In order to treat the general case of a monotone flux, we will first treat the case of a Godunov one. The case of the Lax-Friedrichs flux will follow easily since it can be split into the sum ot two monotone fluxes, which are particular cases of Godunov fluxes. From now on we will hence suppose that $F$ is a Godunov flux and at the end of the proof we will extend it to the general case of a monotone one, following the idea presented above. As we will see later, we will only exploit the fact that the flux is a Godunov one in some parts of the proof of Proposition 5.3: precisely to show that $B_{1}^{h, k}-B_{2}^{h, k} \geqslant 0$ in Step I.2.1, that $B_{2, b}^{h, k}-\tilde{B}_{3, b}^{h, k} \geqslant 0$ in Step I.2.2 and that $B_{2, \text { int }}^{h, k}-B_{3, \text { int }}^{h, k}$ goes to 0 in Step II.2.

We propose in this section entropy inequalities satisfied by the finite volume approximate solution and aim to pass to the limit in these formulations in order to show the convergence of the scheme. For technical reason, one considers a time-continuous approximate solution constructed from $u_{\mathcal{T}, k}$ and denoted $\bar{u}_{\mathcal{T}, k}$ in the sequel.

\subsection{A time-continuous approximation}

Set $K \in \mathcal{T}, n \in\{0, \ldots, N-1\}$ and consider $\bar{u}_{K, n}(s)$ the time-continuous stochastic process defined on $\Omega \times[n k,(n+1) k)$ from the discrete unknowns $u_{K}^{n}$ by:

$$
\begin{aligned}
\bar{u}_{K, n}(s) & =u_{K}^{n}-\int_{n k}^{s} \frac{1}{|K|} \sum_{\sigma \in \mathcal{E}_{K}}|\sigma|\left\{v_{K, \sigma}^{n,+} F\left(u_{K}^{n}, u_{K, \sigma}^{n}\right)-v_{K, \sigma}^{n,-} F\left(u_{K, \sigma}^{n}, u_{K}^{n}\right)\right\} \mathrm{d} t+\int_{n k}^{s} g\left(u_{K}^{n}\right) \mathrm{d} W(t) \\
& =u_{K}^{n}-\frac{s-n k}{|K|} \sum_{\sigma \in \mathcal{E}_{K}}|\sigma|\left\{v_{K, \sigma}^{n,+} F\left(u_{K}^{n}, u_{K, \sigma}^{n}\right)-v_{K, \sigma}^{n,-} F\left(u_{K, \sigma}^{n}, u_{K}^{n}\right)\right\}+\int_{n k}^{s} g\left(u_{K}^{n}\right) \mathrm{d} W(t) .
\end{aligned}
$$

In this way, we have for almost all $\omega, \bar{u}_{K, n}(\omega, n k)=u_{K}^{n}$ and $\bar{u}_{K, n}(\omega,(n+1) k)=u_{K}^{n+1}$ and therefore we can now define a time-continuous approximate solution $\bar{u}_{\mathcal{T}, k}$ on $\Omega \times D \times[0, T)$ by

$$
\bar{u}_{\mathcal{T}, k}(\omega, x, t)=\bar{u}_{K, n}(\omega, t), \omega \in \Omega, x \in K \text { and } t \in[n k,(n+1) k] .
$$

Using again the fact that $\sum_{\sigma \in \mathcal{E}_{K}}|\sigma|\left(v_{K, \sigma}^{n,+}-v_{K, \sigma}^{n,-}\right)=0$ we can rewrite for any $K \in \mathcal{T}$ and $n \in\{0, \ldots, N-1\}$ the time-continuous approximate solution $\bar{u}_{\mathcal{T}, k}$ on $\Omega \times K \times[n k,(n+1) k]$ in the following way:

$$
\begin{aligned}
\bar{u}_{K, n}(s)= & u_{K}^{n}-\frac{s-n k}{|K|} \sum_{\sigma \in \mathcal{E}_{K}}|\sigma|\left\{v_{K, \sigma}^{n,+}\left(F\left(u_{K}^{n}, u_{K, \sigma}^{n}\right)-f\left(u_{K}^{n}\right)\right)-v_{K, \sigma}^{n,-}\left(F\left(u_{K, \sigma}^{n}, u_{K}^{n}\right)-f\left(u_{K}^{n}\right)\right)\right\} \\
& +\int_{n k}^{s} g\left(u_{K}^{n}\right) \mathrm{d} W(t) .
\end{aligned}
$$

We now estimate the difference between the continuous approximation $\bar{u}_{\mathcal{T}, k}$ and the finite volume solution $u_{\mathcal{T}, k}$.

Proposition 5.2. Let $\mathcal{T}$ be an admissible mesh in the sense of Definition $3.1, N \in \mathbb{N}^{\star}$ and let $k=\frac{T}{N} \in \mathbb{R}_{+}^{\star}$ satisfying the CFL Condition (4.17). Let $\bar{u}_{\mathcal{T}, k}$ be the time-continuous approximate solution defined by (5.3)(5.4), and $u_{\mathcal{T}, k}$ be the finite volume approximate solution defined by (3.5)-(3.6). Then there exists $c \in \mathbb{R}_{+}^{\star}$ depending only on $T,|D|, V, C_{g}, g(0), F_{1}, F_{2}, \bar{\alpha}, u_{0}$ and $u^{b}$ such that

$$
\left\|u_{\mathcal{T}, k}-\bar{u}_{\mathcal{T}, k}\right\|_{L^{2}(\Omega \times Q)}^{2} \leqslant c(h+k) .
$$


Proof. Using the equivalent definition (5.4) of $\bar{u}_{\mathcal{T}, k}$, the fact that $W(s)-W^{n}$ and $u_{K}^{n}$ are independent, we have

$$
\begin{aligned}
\| u_{\mathcal{T}, k}-\left.\bar{u}_{\mathcal{T}, k}\right|_{L^{2}(\Omega \times Q)} ^{2} & \sum_{K \in \mathcal{T}} \sum_{n=0}^{N-1} \int_{n k}^{(n+1) k} \int_{K} E\left[\left(g\left(u_{K}^{n}\right)\left(W(s)-W^{n}\right)\right.\right. \\
& \left.\left.+\frac{s-n k}{|K|} \sum_{\sigma \in \mathcal{E}_{K}}|\sigma|\left\{v_{K, \sigma}^{n,+}\left(F\left(u_{K}^{n}, u_{K, \sigma}^{n}\right)-f\left(u_{K}^{n}\right)\right)-v_{K, \sigma}^{n,-}\left(F\left(u_{K, \sigma}^{n}, u_{K}^{n}\right)-f\left(u_{K}^{n}\right)\right)\right\}\right)^{2}\right] \mathrm{d} x \mathrm{~d} s \\
= & \sum_{K \in \mathcal{T}} \sum_{n=0}^{N-1} \int_{n k}^{(n+1) k}\left\{|K| E\left[\left(g\left(u_{K}^{n}\right)\left(W^{n}-W(s)\right)\right)^{2}\right]\right. \\
& \left.+|K| E\left[\left(\frac{s-n k}{|K|} \sum_{\sigma \in \mathcal{E}_{K}}|\sigma|\left\{v_{K, \sigma}^{n,+}\left(F\left(u_{K}^{n}, u_{K, \sigma}^{n}\right)-f\left(u_{K}^{n}\right)\right)-v_{K, \sigma}^{n,-}\left(F\left(u_{K, \sigma}^{n}, u_{K}^{n}\right)-f\left(u_{K}^{n}\right)\right)\right\}\right)^{2}\right]\right\} \mathrm{d} s \\
\leqslant & \sum_{K \in \mathcal{T}} \sum_{n=0}^{N-1}|K| k^{2} 2\left(g^{2}(0)+C_{g}^{2} E\left[\left(u_{K}^{n}\right)^{2}\right]\right) \\
& +\sum_{K \in \mathcal{T}} \sum_{n=0}^{N-1} k \frac{k^{2}|K|}{|K|^{2}} E\left[\left(\sum_{\sigma \in \mathcal{E}_{K}}|\sigma|\left\{v_{K, \sigma}^{n,+}\left(F\left(u_{K}^{n}, u_{K, \sigma}^{n}\right)-f\left(u_{K}^{n}\right)\right)-v_{K, \sigma}^{n,-}\left(F\left(u_{K, \sigma}^{n}, u_{K}^{n}\right)-f\left(u_{K}^{n}\right)\right)\right\}\right)^{2}\right] .
\end{aligned}
$$

We use now Cauchy-Schwarz inequality (similarly to the proof of inequality (4.6)), the consequence of the assumptions on the mesh (3.2), the CFL condition (4.1) and then the first estimate given in Proposition 4.3 (note that we can apply this proposition since the more restrictive CFL condition (4.17) is fulfilled):

$$
\begin{aligned}
\left\|u_{\mathcal{T}, k}-\bar{u}_{\mathcal{T}, k}\right\|_{L^{2}(\Omega \times Q)}^{2} \leqslant & 2 k C_{g}^{2}\left\|u_{\mathcal{T}, k}\right\|_{L^{2}(\Omega \times Q)}^{2}+2 k T|D| g^{2}(0) \\
& +\sum_{n=0}^{N-1} k \sum_{K \in \mathcal{T}} \sum_{\sigma \in \mathcal{E}_{K}} k^{2} V \frac{|\partial K|}{|K|}|\sigma| E \\
& \times\left[v_{K, \sigma}^{n,+}\left(F\left(u_{K}^{n}, u_{K, \sigma}^{n}\right)-f\left(u_{K}^{n}\right)\right)^{2}+v_{K, \sigma}^{n,-}\left(F\left(u_{K, \sigma}^{n}, u_{K}^{n}\right)-f\left(u_{K}^{n}\right)\right)^{2}\right] \\
\leqslant & 2 k T\left(C_{g}^{2} C_{e s t}^{2}+|D| g^{2}(0)\right)+C_{1} \frac{\bar{\alpha}^{2} h}{V\left(F_{1}+F_{2}\right)^{2}},
\end{aligned}
$$

where we have used the constants $C_{\text {est }}$ and $C_{1}$ given respectively by Propositions 4.1 and 4.3.

\subsection{Entropy inequalities for the approximate solutions}

In this section, entropy inequalities satisfied by the approximate solutions are introduced (Prop. 5.5), and will be used in the proof of convergence of the numerical scheme (Thm. 5.6). In order to obtain these entropy inequalities, some discrete entropy inequalities satisfied by the approximate solution are first derived in the following proposition.

For all $(a, b) \in \mathbb{R}^{2}$ we will denote in the sequel by $s(a, b) \in[\min (a, b), \max (a, b)]$ a real such that $F(a, b)=$ $f(s(a, b))$. We define then the associated numerical entropy flux $G$ by $G(a, b)=\Phi(s(a, b))$ for any $a, b \in \mathbb{R}$ which satisfies for all $a \in \mathbb{R}, G(a, a)=\Phi(a)$. Let us mention that we exploit here the fact that the numerical flux is a Godunov flux to get the existence of $s(a, b)$ and therefore to define the numerical entropy flux $\mathrm{G}$.

Proposition 5.3 (Discrete entropy inequalities). Assume that hypotheses $H_{1}$ to $H_{7}$ hold and that $F$ is the Godunov flux defined by (5.1). Let $\mathcal{T}$ be an admissible mesh in the sense of Definition $3.1, N \in \mathbb{N}^{\star}$ and let 
$k=\frac{T}{N} \in \mathbb{R}_{+}^{\star}$ be the time step. Then $P$-a.s in $\Omega$, for any $\eta \in \mathcal{A}$ and for any $\varphi \in \mathcal{D}^{+}\left(\mathbb{R}^{d} \times[0, T)\right)$ :

$$
\begin{array}{r}
-\sum_{n=0}^{N-1} \sum_{K \in \mathcal{T}} \int_{K}\left(\eta\left(u_{K}^{n+1}\right)-\eta\left(u_{K}^{n}\right)\right) \varphi(x, n k) \mathrm{d} x+\sum_{n=0}^{N-1} \sum_{K \in \mathcal{T}} \int_{n k}^{(n+1) k} \int_{K} \Phi\left(u_{K}^{n}\right) \boldsymbol{v}(x, t) . \nabla_{x} \varphi(x, n k) \mathrm{d} x \mathrm{~d} t \\
+\sum_{n=0}^{N-1} \sum_{K \in \mathcal{T}} \int_{n k}^{(n+1) k} \int_{K} \eta^{\prime}\left(u_{K}^{n}\right) g\left(u_{K}^{n}\right) \varphi(x, n k) \mathrm{d} x \mathrm{~d} W(t)+\frac{1}{2} \sum_{n=0}^{N-1} \sum_{K \in \mathcal{T}} \int_{n k}^{(n+1) k} \int_{K} \eta^{\prime \prime}\left(u_{K}^{n}\right) g^{2}\left(u_{K}^{n}\right) \varphi(x, n k) \mathrm{d} x \mathrm{~d} t \\
+C_{f} V \sum_{n=0}^{N-1} \sum_{\sigma \in \mathcal{E}^{b}} \int_{n k}^{(n+1) k} \int_{\sigma} \eta\left(u^{b}(x, t)\right) \varphi(x, n k) \mathrm{d} \gamma(x) \mathrm{d} t \geqslant R^{h, k}
\end{array}
$$

where for any P-measurable set $A, E\left[\mathbb{1}_{A} R^{h, k}\right] \rightarrow 0$ as $\left(h, \frac{k}{h}\right) \rightarrow(0,0)$.

Proof. In order to prove this proposition, we are going to show firstly that inequality (5.5) holds for a convenient $R^{h, k}$ and in a second time, we will prove that for any $P$-measurable set $A, E\left[\mathbb{1}_{A} R^{h, k}\right] \rightarrow 0$ as $\left(h, \frac{k}{h}\right) \rightarrow(0,0)$. We will in particular use some technics from $[8,13,22]$ and adapt them to our case.

Let $\mathcal{T}$ be an admissible mesh in the sense of Definition 3.1, $N \in \mathbb{N}^{\star}$ and $k=\frac{T}{N} \in \mathbb{R}_{+}^{\star}$. Consider $\eta \in \mathcal{A}$ and $\varphi \in \mathcal{D}^{+}\left(\mathbb{R}^{d} \times[0, T)\right)$. Recall that there exists $\kappa \in \mathbb{R}$ such that for any $t \neq \kappa, \eta(t)>\eta(\kappa)=0$. We assume that $(h, k / h) \rightarrow(0,0)$, in this way we can suppose that the CFL Condition

$$
k \leqslant \frac{(1-\xi) \bar{\alpha}^{2} h}{\left(F_{1}+F_{2}\right) V}
$$

holds for some $\xi \in(0,1)$. In this manner, the estimates given by Proposition 4.1 and Proposition 4.3 hold.

Step I: Existence of $R_{h, k}$

The application of Itô's formula to the time-continuous process $\bar{u}_{K, n}$ defined by equation (5.2) for some $K \in \mathcal{T}$ and the function $v \in \mathbb{R} \mapsto \eta(v) \in \mathbb{R}$ on the interval $[n k,(n+1) k]$ yields P-a.s in $\Omega$

$$
\begin{aligned}
\eta\left(\bar{u}_{K, n}((n+1) k)\right)= & \eta\left(\bar{u}_{K, n}(n k)\right)-\frac{1}{|K|} \int_{n k}^{(n+1) k} \eta^{\prime}\left(\bar{u}_{\mathcal{T}, k}(t)\right) \sum_{\sigma \in \mathcal{E}_{K}}|\sigma|\left\{v_{K, \sigma}^{n,+} F\left(u_{K}^{n}, u_{K, \sigma}^{n}\right)-v_{K, \sigma}^{n,-} F\left(u_{K, \sigma}^{n}, u_{K}^{n}\right)\right\} \mathrm{d} t \\
& +\int_{n k}^{(n+1) k} \eta^{\prime}\left(\bar{u}_{\mathcal{T}, k}(t)\right) g\left(u_{K}^{n}\right) \mathrm{d} W(t)+\frac{1}{2} \int_{n k}^{(n+1) k} \eta^{\prime \prime}\left(\bar{u}_{\mathcal{T}, k}(t)\right) g^{2}\left(u_{K}^{n}\right) \mathrm{d} t .
\end{aligned}
$$

Let us multiply equation (5.6) by $|K| \varphi_{K}^{n}$, where $\varphi_{K}^{n}=\frac{1}{|K|} \int_{K} \varphi(x, n k) \mathrm{d} x$, and sum for all $K \in \mathcal{T}$ and $n \in$ $\{0, \ldots, N-1\}$. One gets P-a.s in $\Omega$

$$
\begin{aligned}
\sum_{n=0}^{N-1} \sum_{K \in \mathcal{T}}\left[\eta\left(u_{K}^{n+1}\right)-\eta\left(u_{K}^{n}\right)\right]|K| \varphi_{K}^{n}= & -\sum_{n=0}^{N-1} \sum_{K \in \mathcal{T}} \int_{n k}^{(n+1) k} \eta^{\prime}\left(\bar{u}_{\mathcal{T}, k}(t)\right) \\
& \times \sum_{\sigma \in \mathcal{E}_{K}}|\sigma|\left\{v_{K, \sigma}^{n,+} F\left(u_{K}^{n}, u_{K, \sigma}^{n}\right)-v_{K, \sigma}^{n,-} F\left(u_{K, \sigma}^{n}, u_{K}^{n}\right)\right\} \mathrm{d} t \varphi_{K}^{n} \\
& +\sum_{n=0}^{N-1} \sum_{K \in \mathcal{T}} \int_{n k}^{(n+1) k} \eta^{\prime}\left(\bar{u}_{\mathcal{T}, k}(t)\right) g\left(u_{K}^{n}\right) \mathrm{d} W(t)|K| \varphi_{K}^{n} \\
& +\frac{1}{2} \sum_{n=0}^{N-1} \sum_{K \in \mathcal{T}} \int_{n k}^{(n+1) k} \eta^{\prime \prime}\left(\bar{u}_{\mathcal{T}, k}(t)\right) g^{2}\left(u_{K}^{n}\right) \mathrm{d} t|K| \varphi_{K}^{n} .
\end{aligned}
$$


This can be written as $A^{h, k}=-B^{h, k}+C^{h, k}+D^{h, k}$, where

$$
\begin{aligned}
A^{h, k} & =\sum_{n=0}^{N-1} \sum_{K \in \mathcal{T}}\left[\eta\left(u_{K}^{n+1}\right)-\eta\left(u_{K}^{n}\right)\right]|K| \varphi_{K}^{n} \\
B^{h, k} & =\sum_{n=0}^{N-1} \sum_{K \in \mathcal{T}} \int_{n k}^{(n+1) k} \eta^{\prime}\left(\bar{u}_{\mathcal{T}, k}(t)\right) \sum_{\sigma \in \mathcal{E}_{K}}|\sigma|\left\{v_{K, \sigma}^{n,+} F\left(u_{K}^{n}, u_{K, \sigma}^{n}\right)-v_{K, \sigma}^{n,-} F\left(u_{K, \sigma}^{n}, u_{K}^{n}\right)\right\} \mathrm{d} t \varphi_{K}^{n} \\
C^{h, k} & =\sum_{n=0}^{N-1} \sum_{K \in \mathcal{T}} \int_{n k}^{(n+1) k} \eta^{\prime}\left(\bar{u}_{\mathcal{T}, k}(t)\right) g\left(u_{K}^{n}\right) \mathrm{d} W(t)|K| \varphi_{K}^{n} \\
D^{h, k} & =\frac{1}{2} \sum_{n=0}^{N-1} \sum_{K \in \mathcal{T}} \int_{n k}^{(n+1) k} \eta^{\prime \prime}\left(\bar{u}_{\mathcal{T}, k}(t)\right) g^{2}\left(u_{K}^{n}\right) \mathrm{d} t|K| \varphi_{K}^{n} .
\end{aligned}
$$

Let us analyze separately these terms.

I.1 Study of $A^{h, k}$ : we note that $-A^{h, k}$ is equal to the first left hand side term of inequality (5.5).

I.2 Study of $B^{h, k}$ : we decompose

$$
B^{h, k}=\sum_{n=0}^{N-1} \sum_{K \in \mathcal{T}} \int_{n k}^{(n+1) k} \frac{1}{|K|} \int_{K} \eta^{\prime}\left(\bar{u}_{\mathcal{T}, k}(t)\right) \sum_{\sigma \in \mathcal{E}_{K}}|\sigma|\left\{v_{K, \sigma}^{n,+} F\left(u_{K}^{n}, u_{K, \sigma}^{n}\right)-v_{K, \sigma}^{n,-} F\left(u_{K, \sigma}^{n}, u_{K}^{n}\right)\right\} \varphi(x, n k) \mathrm{d} x \mathrm{~d} t
$$

in the following way

$$
B^{h, k}=B^{h, k}-B_{1}^{h, k}+B_{1}^{h, k}-B_{2}^{h, k}+B_{2}^{h, k}-B_{3}^{h, k}+B_{3}^{h, k},
$$

where

$$
\begin{aligned}
B_{1}^{h, k}= & \sum_{n=0}^{N-1} \sum_{K \in \mathcal{T}} \int_{n k}^{(n+1) k} \frac{1}{|K|} \int_{K} \eta^{\prime}\left(u_{K}^{n}\right) \sum_{\sigma \in \mathcal{E}_{K}}|\sigma|\left\{v_{K, \sigma}^{n,+} F\left(u_{K}^{n}, u_{K, \sigma}^{n}\right)-v_{K, \sigma}^{n,-} F\left(u_{K, \sigma}^{n}, u_{K}^{n}\right)\right\} \varphi(x, n k) \mathrm{d} x \mathrm{~d} t \\
B_{2}^{h, k}= & \sum_{n=0}^{N-1} \sum_{K \in \mathcal{T}} \int_{n k}^{(n+1) k} \frac{1}{|K|} \int_{K} \sum_{\sigma \in \mathcal{E}_{K}}|\sigma|\left\{v_{K, \sigma}^{n,+} G\left(u_{K}^{n}, u_{K, \sigma}^{n}\right)-v_{K, \sigma}^{n,-} G\left(u_{K, \sigma}^{n}, u_{K}^{n}\right)\right\} \varphi(x, n k) \mathrm{d} x \mathrm{~d} t \\
B_{3}^{h, k}= & -\sum_{n=0}^{N-1} \sum_{K \in \mathcal{T}} \int_{n k}^{(n+1) k} \int_{K} \Phi\left(u_{K}^{n}\right) \boldsymbol{v}(x, t) . \nabla_{x} \varphi(x, n k) \mathrm{d} x \mathrm{~d} t \\
& -C_{f} V \sum_{n=0}^{N-1} \sum_{\sigma \in \mathcal{E}^{b}} \int_{n k}^{(n+1) k} \int_{\sigma} \eta\left(u^{b}(x, t)\right) \varphi(x, n k) \mathrm{d} \gamma(x) \mathrm{d} t .
\end{aligned}
$$

I.2.1 Study of $B_{1}^{h, k}-B_{2}^{h, k}$ : we show that P-almost surely in $\Omega, B_{1}^{h, k}-B_{2}^{h, k} \geqslant 0$.

Firstly, we notice that by (3.8), we have

$$
\sum_{\sigma \in \mathcal{E}_{K}}|\sigma|\left(v_{K, \sigma}^{n,+}-v_{K, \sigma}^{n,-}\right) \Phi\left(u_{K}^{n}\right)=0 \text { and } \sum_{\sigma \in \mathcal{E}_{K}}|\sigma|\left(v_{K, \sigma}^{n,+}-v_{K, \sigma}^{n,-}\right) f\left(u_{K}^{n}\right)=0 .
$$


Secondly, recall that for any $K \in \mathcal{T}, F\left(u_{K}^{n}, u_{K}^{n}\right)=f\left(u_{K}^{n}\right)$ and $G\left(u_{K}^{n}, u_{K}^{n}\right)=\Phi\left(u_{K}^{n}\right)$ and that $\Phi$ is defined by $\Phi(a)=\int_{\kappa}^{a} \eta^{\prime}(t) f^{\prime}(t) \mathrm{d} t, \forall a \in \mathbb{R}$. Hence we can rewrite $B_{1}^{h, k}-B_{2}^{h, k}$ in the following way:

$$
\begin{aligned}
B_{1}^{h, k}-B_{2}^{h, k}= & \sum_{n=0}^{N-1} \sum_{K \in \mathcal{T}} \frac{k}{|K|} \sum_{\sigma \in \mathcal{E}_{K}}|\sigma|\left\{v_{K, \sigma}^{n,+}\left[\eta^{\prime}\left(u_{K}^{n}\right)\left(F\left(u_{K}^{n}, u_{K, \sigma}^{n}\right)-f\left(u_{K}^{n}\right)\right)-\left(G\left(u_{K}^{n}, u_{K, \sigma}^{n}\right)-\Phi\left(u_{K}^{n}\right)\right)\right]\right. \\
& \left.-v_{K, \sigma}^{n,-}\left[\eta^{\prime}\left(u_{K}^{n}\right)\left(F\left(u_{K, \sigma}^{n}, u_{K}^{n}\right)-f\left(u_{K}^{n}\right)\right)-\left(G\left(u_{K, \sigma}^{n}, u_{K}^{n}\right)-\Phi\left(u_{K}^{n}\right)\right)\right]\right\} \int_{K} \varphi(x, n k) \mathrm{d} x .
\end{aligned}
$$

Let $K \in \mathcal{T}, \sigma \in \mathcal{E}_{K}$ and suppose that $u_{K}^{n}<u_{K, \sigma}^{n}$ (note that the case $u_{K, \sigma}^{n}<u_{K}^{n}$ is similar).

We first determine the sign of

$$
\eta^{\prime}\left(u_{K}^{n}\right)\left(F\left(u_{K}^{n}, u_{K, \sigma}^{n}\right)-f\left(u_{K}^{n}\right)\right)-\left(G\left(u_{K}^{n}, u_{K, \sigma}^{n}\right)-\Phi\left(u_{K}^{n}\right)\right) .
$$

Using the fact that we are in the particular case where $F$ is the Godunov numerical flux, we know that there exists $s\left(u_{K}^{n}, u_{K, \sigma}^{n}\right) \in\left[u_{K}^{n}, u_{K, \sigma}^{n}\right]$ such that

$$
F\left(u_{K}^{n}, u_{K, \sigma}^{n}\right)=f\left(s\left(u_{K}^{n}, u_{K, \sigma}^{n}\right)\right)=\min _{t \in\left[u_{K}^{n}, u_{K, \sigma}^{n}\right]} f(t) .
$$

In this way,

$$
\begin{aligned}
\eta^{\prime} & \left(u_{K}^{n}\right)\left(F\left(u_{K}^{n}, u_{K, \sigma}^{n}\right)-f\left(u_{K}^{n}\right)\right)-\left(G\left(u_{K}^{n}, u_{K, \sigma}^{n}\right)-\Phi\left(u_{K}^{n}\right)\right) \\
= & \eta^{\prime}\left(u_{K}^{n}\right)\left(f\left(s\left(u_{K}^{n}, u_{K, \sigma}^{n}\right)\right)-f\left(u_{K}^{n}\right)\right)-\left(\Phi\left(s\left(u_{K}^{n}, u_{K, \sigma}^{n}\right)\right)-\Phi\left(u_{K}^{n}\right)\right) \\
= & \int_{u_{K}^{n}}^{s\left(u_{K}^{n}, u_{K, \sigma}^{n}\right)} f^{\prime}(t) \eta^{\prime}\left(u_{K}^{n}\right) \mathrm{d} t-\int_{u_{K}^{n}}^{s\left(u_{K}^{n}, u_{K, \sigma}^{n}\right)} f^{\prime}(t) \eta^{\prime}(t) \mathrm{d} t \\
= & \int_{u_{K}^{n}}^{s\left(u_{K}^{n}, u_{K, \sigma}^{n}\right)} f^{\prime}(t)\left(\eta^{\prime}\left(u_{K}^{n}\right)-\eta^{\prime}(t)\right) \mathrm{d} t \\
= & \int_{u_{K}^{n}}^{s\left(u_{K}^{n}, u_{K, \sigma}^{n}\right)} f(t) \eta^{\prime \prime}(t) \mathrm{d} t+f\left(s\left(u_{K}^{n}, u_{K, \sigma}^{n}\right)\right)\left\{\eta^{\prime}\left(u_{K}^{n}\right)-\eta^{\prime}\left(s\left(u_{K}^{n}, u_{K, \sigma}^{n}\right)\right)\right\} \\
\geqslant & \int_{u_{K}^{n}}^{s\left(u_{K}^{n}, u_{K, \sigma}^{n}\right)} f\left(s\left(u_{K}^{n}, u_{K, \sigma}^{n}\right)\right) \eta^{\prime \prime}(t) \mathrm{d} t+f\left(s\left(u_{K}^{n}, u_{K, \sigma}^{n}\right)\right)\left\{\eta^{\prime}\left(u_{K}^{n}\right)-\eta^{\prime}\left(s\left(u_{K}^{n}, u_{K, \sigma}^{n}\right)\right)\right\} \\
= & 0 .
\end{aligned}
$$

Using the same technics, we get

$$
\eta^{\prime}\left(u_{K}^{n}\right)\left(F\left(u_{K, \sigma}^{n}, u_{K}^{n}\right)-f\left(u_{K}^{n}\right)\right)-\left(G\left(u_{K, \sigma}^{n}, u_{K}^{n}\right)-\Phi\left(u_{K}^{n}\right)\right) \leqslant 0 .
$$

Finally we obtain the following inequality

$$
\begin{aligned}
& v_{K, \sigma}^{n,+}\left[\eta^{\prime}\left(u_{K}^{n}\right)\left(F\left(u_{K}^{n}, u_{K, \sigma}^{n}\right)-f\left(u_{K}^{n}\right)\right)-\left(G\left(u_{K}^{n}, u_{K, \sigma}^{n}\right)-\Phi\left(u_{K}^{n}\right)\right)\right] \\
- & v_{K, \sigma}^{n,-}\left[\eta^{\prime}\left(u_{K}^{n}\right)\left(F\left(u_{K, \sigma}^{n}, u_{K}^{n}\right)-f\left(u_{K}^{n}\right)\right)-\left(G\left(u_{K, \sigma}^{n}, u_{K}^{n}\right)-\Phi\left(u_{K}^{n}\right)\right)\right] \geqslant 0,
\end{aligned}
$$

which allows us to deduce that for almost all $\omega \in \Omega$,

$$
B_{1}^{h, k}-B_{2}^{h, k} \geqslant 0 \text {. }
$$


I.2.2 Study of $B_{2}^{h, k}-B_{3}^{h, k}$ : Using again (3.8), we get

$$
\sum_{\sigma \in \mathcal{E}_{K}}|\sigma|\left(v_{K, \sigma}^{n,+}-v_{K, \sigma}^{n,-}\right) \Phi\left(u_{K}^{n}\right)=0
$$

we can rewrite $B_{2}^{h, k}$ in the following way

$$
\begin{aligned}
B_{2}^{h, k}= & \sum_{n=0}^{N-1} \sum_{K \in \mathcal{T}} \frac{k}{|K|} \sum_{\sigma \in \mathcal{E}_{K}}|\sigma|\left\{v_{K, \sigma}^{n,+}\left(G\left(u_{K}^{n}, u_{K, \sigma}^{n}\right)-\Phi\left(u_{K}^{n}\right)\right)-v_{K, \sigma}^{n,-}\left(G\left(u_{K, \sigma}^{n}, u_{K}^{n}\right)-\Phi\left(u_{K}^{n}\right)\right)\right\} \\
& \times \int_{K} \varphi(x, n k) \mathrm{d} x .
\end{aligned}
$$

By separating this summation by edges belonging respectively to the interior of the domain $D$ and respectively to the boundary of the domain $D$ (see formula (4.7)), we get

$$
B_{2}^{h, k}=B_{2, i n t}^{h, k}+B_{2, b}^{h, k},
$$

where

$$
\begin{aligned}
B_{2, \text { int }}^{h, k}= & S_{2, \text { int }}^{h, k}-T_{2, \text { int }}^{h, k}, \text { with } \\
S_{2, \text { int }}^{h, k}= & \sum_{n=0}^{N} \sum_{(K, L) \in \mathfrak{T}_{n}} \frac{k}{|K|}\left|\sigma_{K, L}\right|\left\{v_{K, L}^{n,+}\left(G\left(u_{K}^{n}, u_{L}^{n}\right)-\Phi\left(u_{K}^{n}\right)\right)-v_{K, L}^{n,-}\left(G\left(u_{L}^{n}, u_{K}^{n}\right)-\Phi\left(u_{K}^{n}\right)\right)\right\} \\
& \times \int_{K} \varphi(x, n k) \mathrm{d} x, \\
T_{2, \text { int }}^{h, k}= & \sum_{n=0}^{N} \sum_{(K, L) \in \mathfrak{T}_{n}} \frac{k}{|L|}\left|\sigma_{K, L}\right|\left\{v_{K, L}^{n,+}\left(G\left(u_{K}^{n}, u_{L}^{n}\right)-\Phi\left(u_{L}^{n}\right)\right)-v_{K, L}^{n,-}\left(G\left(u_{L}^{n}, u_{K}^{n}\right)-\Phi\left(u_{L}^{n}\right)\right)\right\} \\
& \times \int_{L} \varphi(x, n k) \mathrm{d} x, \\
B_{2, b}^{h, k}= & \sum_{n=0}^{N} \sum_{\sigma \in \mathcal{E}^{b}} \frac{k}{|K|}|\sigma|\left\{v_{K, \sigma}^{n,+}\left(G\left(u_{K}^{n}, u_{\sigma}^{b, n}\right)-\Phi\left(u_{K}^{n}\right)\right)-v_{K, \sigma}^{n,-}\left(G\left(u_{\sigma}^{b, n}, u_{K}^{n}\right)-\Phi\left(u_{K}^{n}\right)\right)\right\} \int_{K} \varphi(x, n k) \mathrm{d} x,
\end{aligned}
$$

where we remind to the reader the definition of the set

$$
\mathfrak{T}_{n}=\left\{(K, L) \in \mathcal{T}^{2}: L \in \mathcal{N}(K) \text { and } u_{K}^{n}>u_{L}^{n}\right\} .
$$

Similarly, since

$$
\begin{aligned}
& -\sum_{n=0}^{N-1} \sum_{K \in \mathcal{T}} \int_{n k}^{(n+1) k} \int_{K} \Phi\left(u_{K}^{n}\right) \boldsymbol{v}(x, t) \cdot \nabla_{x} \varphi(x, n k) \mathrm{d} x \mathrm{~d} t \\
& =-\sum_{n=0}^{N-1} \sum_{K \in \mathcal{T}} \int_{n k}^{(n+1) k} \int_{K} \Phi\left(u_{K}^{n}\right) \operatorname{div}[\boldsymbol{v}(x, t) \varphi(x, n k)] \mathrm{d} x \mathrm{~d} t \\
& =-\sum_{n=0}^{N-1} \sum_{K \in \mathcal{T}} \sum_{\sigma \in \mathcal{E}_{K}} \int_{n k}^{(n+1) k} \int_{\sigma} \Phi\left(u_{K}^{n}\right) \boldsymbol{v}(x, t) \cdot n_{K, \sigma} \varphi(x, n k) \mathrm{d} \gamma(x) \mathrm{d} t
\end{aligned}
$$

we have

$$
B_{3}^{h, k}=B_{3, i n t}^{h, k}+B_{3, b}^{h, k},
$$


where $B_{3, \text { int }}^{h, k}=S_{3, \text { int }}^{h, k}-T_{3, \text { int }}^{h, k}$, with

$$
\begin{aligned}
S_{3, i n t}^{h, k}= & \sum_{n=0}^{N-1} \sum_{(K, L) \in \mathfrak{T}_{n}} \int_{n k}^{(n+1) k} \int_{\sigma_{K, L}}\left\{v_{K, L}^{n,+} G\left(u_{K}^{n}, u_{L}^{n}\right)-v_{K, L}^{n,-} G\left(u_{L}^{n}, u_{K}^{n}\right)\right. \\
& \left.-\Phi\left(u_{K}^{n}\right) \boldsymbol{v}(x, t) \cdot n_{K, L}\right\} \varphi(x, n k) \mathrm{d} \gamma(x) \mathrm{d} t, \\
T_{3, i n t}^{h, k}= & \sum_{n=0}^{N-1} \sum_{(K, L) \in \mathfrak{T}_{n}} \int_{n k}^{(n+1) k} \int_{\sigma_{K, L}}\left\{v_{K, L}^{n,+} G\left(u_{K}^{n}, u_{L}^{n}\right)-v_{K, L}^{n,-} G\left(u_{L}^{n}, u_{K}^{n}\right)\right. \\
& \left.-\Phi\left(u_{L}^{n}\right) \boldsymbol{v}(x, t) \cdot n_{K, L}\right\} \varphi(x, n k) \mathrm{d} \gamma(x) \mathrm{d} t,
\end{aligned}
$$

and

$$
\begin{aligned}
B_{3, b}^{h, k}= & -\sum_{n=0}^{N-1} \sum_{\sigma \in \mathcal{E}^{b}} \int_{n k}^{(n+1) k} \int_{\sigma} \boldsymbol{v}(x, t) \cdot n_{K, \sigma} \Phi\left(u_{K}^{n}\right) \varphi(x, n k) \mathrm{d} \gamma(x) \mathrm{d} t \\
& -C_{f} V \sum_{n=0}^{N-1} \sum_{\sigma \in \mathcal{E}^{b}} \int_{n k}^{(n+1) k} \int_{\sigma} \eta\left(u^{b}(x, t)\right) \varphi(x, n k) \mathrm{d} \gamma(x) \mathrm{d} t .
\end{aligned}
$$

Using these notations, we will be able to show that for almost all $\omega \in \Omega$,

$$
B_{2}^{h, k}-B_{3}^{h, k} \geqslant B_{2, \text { int }}^{h, k}-B_{3, \text { int }}^{h, k}+\tilde{B}_{3, b}^{h, k}-B_{3, b}^{h, k},
$$

where

$$
\tilde{B}_{3, b}^{h, k}=-\sum_{n=0}^{N-1} \sum_{\sigma \in \mathcal{E}^{b}} \frac{k}{|K|}|\sigma|\left\{\left(v_{K, \sigma}^{n,+}-v_{K, \sigma}^{n,-}\right) \Phi\left(u_{K}^{n}\right)+C_{f} V \eta\left(u_{\sigma}^{b, n}\right)\right\} \int_{K} \varphi(x, n k) \mathrm{d} x .
$$

Since

$$
B_{2}^{h, k}-B_{3}^{h, k}=B_{2, \text { int }}^{h, k}-B_{3, \text { int }}^{h, k}+B_{2, b}^{h, k}-\tilde{B}_{3, b}^{h, k}+\tilde{B}_{3, b}^{h, k}-B_{3, b}^{h, k},
$$

it remains to show that for almost all $\omega \in \Omega$,

$$
B_{2, b}^{h, k}-\tilde{B}_{3, b}^{h, k} \geqslant 0
$$

We have

$$
B_{2, b}^{h, k}-\tilde{B}_{3, b}^{h, k}=\sum_{n=0}^{N-1} \sum_{\sigma \in \mathcal{E}^{b}} \frac{k}{|K|}|\sigma|\left\{v_{K, \sigma}^{n,+} G\left(u_{K}^{n}, u_{\sigma}^{b, n}\right)-v_{K, \sigma}^{n,-} G\left(u_{\sigma}^{b, n}, u_{K}^{n}\right)+C_{f} V \eta\left(u_{\sigma}^{b, n}\right)\right\} \int_{K} \varphi(x, n k) \mathrm{d} x .
$$

Since $\varphi$ is a nonnegative test function, it remains to show that for any $n \in\{0, \ldots, N\}$ and any $\sigma \in \partial K \cap \mathcal{E}^{b}$, we have

$$
v_{K, \sigma}^{n,+} G\left(u_{K}^{n}, u_{\sigma}^{b, n}\right)-v_{K, \sigma}^{n,-} G\left(u_{\sigma}^{b, n}, u_{K}^{n}\right)+C_{f} V \eta\left(u_{\sigma}^{b, n}\right) \geqslant 0 .
$$

To obtain this last inequality, we will exploit the fact that we are in the particular case where $F$ is the Godunov numerical flux. We will split the proof in six cases which correspond to the six possible positions for $u_{K}^{n}$ and $u_{\sigma}^{b, n}$ with respect to the parameter $\kappa(\kappa \in \mathbb{R}$ is the unique minimizer of the entropy $\eta$ over $\mathbb{R}$, it satisfies $\eta(\kappa)=0)$. 
Recall that there exists $s\left(u_{K}^{n}, u_{\sigma}^{b, n}\right)$ and $s\left(u_{\sigma}^{b, n}, u_{K}^{n}\right)$ belonging to $\left[\min \left(u_{K}^{n}, u_{\sigma}^{b, n}\right), \max \left(u_{K}^{n}, u_{\sigma}^{b, n}\right)\right]$ such that

$$
\begin{aligned}
f\left(s\left(u_{K}^{n}, u_{\sigma}^{b, n}\right)\right)=\left\{\begin{array}{l}
\min _{t \in\left[u_{K}^{n}, u_{\sigma}^{b, n}\right]} f(t) \text { if } u_{K}^{n} \leqslant u_{\sigma}^{b, n} \\
\max _{t \in\left[u_{\sigma}^{b, n}, u_{K}^{n}\right]} f(t) \text { if } u_{K}^{n} \geqslant u_{\sigma}^{b, n},
\end{array}\right. \\
f\left(s\left(u_{\sigma}^{b, n}, u_{K}^{n}\right)\right)=\left\{\begin{array}{l}
\max _{t \in\left[u_{K}^{n}, u_{\sigma}^{b, n}\right]} f(t) \text { if } u_{K}^{n} \leqslant u_{\sigma}^{b, n} \\
\min _{t \in\left[u_{\sigma}^{b, n}, u_{K}^{n}\right]} f(t) \text { if } u_{K}^{n} \geqslant u_{\sigma}^{b, n},
\end{array}\right. \\
G\left(u_{K}^{n}, u_{\sigma}^{b, n}\right)=\Phi\left(s\left(u_{K}^{n}, u_{\sigma}^{b, n}\right)\right)=\int_{\kappa}^{s\left(u_{K}^{n}, u_{\sigma}^{b, n}\right)} \eta^{\prime}(t) f^{\prime}(t) \mathrm{d} t,
\end{aligned}
$$

and

$$
G\left(u_{\sigma}^{b, n}, u_{K}^{n}\right)=\Phi\left(s\left(u_{\sigma}^{b, n}, u_{K}^{n}\right)\right)=\int_{\kappa}^{s\left(u_{\sigma}^{b, n}, u_{K}^{n}\right)} \eta^{\prime}(t) f^{\prime}(t) \mathrm{d} t .
$$

We show in the following that whatever the position of $u_{\sigma}^{b, n}$ and $u_{K}^{n}$ with respect to $\kappa$ is, we always have

$$
G\left(u_{K}^{n}, u_{\sigma}^{b, n}\right) \geqslant-C_{f} \eta\left(u_{\sigma}^{b, n}\right) \text { and } G\left(u_{\sigma}^{b, n}, u_{K}^{n}\right) \leqslant C_{f} \eta\left(u_{\sigma}^{b, n}\right) .
$$

As a consequence we get the announced inequality, that is

$$
\begin{aligned}
v_{K, \sigma}^{n,+} G\left(u_{K}^{n}, u_{\sigma}^{b, n}\right)-v_{K, \sigma}^{n,-} G\left(u_{\sigma}^{b, n}, u_{K}^{n}\right) & \geqslant-\left(v_{K, \sigma}^{n,+}+v_{K, \sigma}^{n,-}\right) C_{f} \eta\left(u_{\sigma}^{b, n}\right) \\
& \geqslant-V C_{f} \eta\left(u_{\sigma}^{b, n}\right) .
\end{aligned}
$$

Let us mention that in every cases, the proofs to show that the two previous inequalities hold are due to the three following properties: firstly, the minimization or maximization of the flux function $f$ by $s\left(u_{K}^{n}, u_{\sigma}^{b, n}\right)$ (respectively by $\left.s\left(u_{\sigma}^{b, n}, u_{K}^{n}\right)\right)$ according to the position of $u_{K}^{n}$ and $u_{\sigma}^{b, n}$, secondly the positivity (respectively negativity) of $\eta^{\prime}$ on $] \kappa,+\infty[$ (respectively on ] $-\infty, \kappa[$ ) and thirdly the montony of $\eta$ which is nondecreasing on $] \kappa,+\infty[$ and nonincreasing on $]-\infty, \kappa[$. This last property implies particularly that $\eta$ is nonnegative on $\mathbb{R}$.

Case 1: $u_{K}^{n} \leqslant \kappa \leqslant u_{\sigma}^{b, n}$

Note that if $s\left(u_{K}^{n}, u_{\sigma}^{b, n}\right) \geqslant \kappa$, then

$$
G\left(u_{K}^{n}, u_{\sigma}^{b, n}\right)=\int_{\kappa}^{s\left(u_{K}^{n}, u_{\sigma}^{b, n}\right)} \eta^{\prime}(t) f^{\prime}(t) \mathrm{d} t \geqslant-C_{f} \int_{\kappa}^{u_{\sigma}^{b, n}} \eta^{\prime}(t) \mathrm{d} t=-C_{f} \eta\left(u_{\sigma}^{b, n}\right) .
$$

Else, if $s\left(u_{K}^{n}, u_{\sigma}^{b, n}\right) \leqslant \kappa$, since $\eta \geqslant 0$ on $\mathbb{R}$ we get

$$
\begin{aligned}
G\left(u_{K}^{n}, u_{\sigma}^{b, n}\right) & =\left[\eta^{\prime}(t) f(t)\right]_{\kappa}^{s\left(u_{K}^{n}, u_{\sigma}^{b, n}\right)}-\int_{\kappa}^{s\left(u_{K}^{n}, u_{\sigma}^{b, n}\right)} \eta^{\prime \prime}(t) f(t) \mathrm{d} t \\
& =\eta^{\prime}\left(s\left(u_{K}^{n}, u_{\sigma}^{b, n}\right)\right) f\left(s\left(u_{K}^{n}, u_{\sigma}^{b, n}\right)\right)-\eta^{\prime}(\kappa) f(\kappa)-\int_{\kappa}^{s\left(u_{K}^{n}, u_{\sigma}^{b, n}\right)} \eta^{\prime \prime}(t) f(t) \mathrm{d} t \\
& =\eta^{\prime}\left(s\left(u_{K}^{n}, u_{\sigma}^{b, n}\right)\right) f\left(s\left(u_{K}^{n}, u_{\sigma}^{b, n}\right)\right)+\int_{s\left(u_{K}^{n}, u_{\sigma}^{b, n}\right)}^{\kappa} \eta^{\prime \prime}(t) f(t) \mathrm{d} t \\
& \geqslant \eta^{\prime}\left(s\left(u_{K}^{n}, u_{\sigma}^{b, n}\right)\right) f\left(s\left(u_{K}^{n}, u_{\sigma}^{b, n}\right)\right)+f\left(s\left(u_{K}^{n}, u_{\sigma}^{b, n}\right)\right) \int_{s\left(u_{K}^{n}, u_{\sigma}^{b, n}\right)}^{\kappa} \eta^{\prime \prime}(t) \mathrm{d} t \\
& =0 \\
& \geqslant-C_{f} \eta\left(u_{\sigma}^{b, n}\right) .
\end{aligned}
$$


Moreover, if $s\left(u_{\sigma}^{b, n}, u_{K}^{n}\right) \geqslant \kappa$

$$
G\left(u_{\sigma}^{b, n}, u_{K}^{n}\right)=\int_{\kappa}^{s\left(u_{\sigma}^{b, n}, u_{K}^{n}\right)} \eta^{\prime}(t) f^{\prime}(t) \mathrm{d} t \leqslant C_{f} \int_{\kappa}^{u_{\sigma}^{b, n}} \eta^{\prime}(t) \mathrm{d} t=C_{f} \eta\left(u_{\sigma}^{b, n}\right) .
$$

And if $s\left(u_{\sigma}^{b, n}, u_{K}^{n}\right) \leqslant \kappa$, using again $\eta \geqslant 0$ on $\mathbb{R}$ we obtain

$$
\begin{aligned}
G\left(u_{\sigma}^{b, n}, u_{K}^{n}\right) & =\left[\eta^{\prime}(t) f(t)\right]_{\kappa}^{s\left(u_{\sigma}^{b, n}, u_{K}^{n}\right)}-\int_{\kappa}^{s\left(u_{\sigma}^{b, n}, u_{K}^{n}\right)} \eta^{\prime \prime}(t) f(t) \mathrm{d} t \\
& =\eta^{\prime}\left(s\left(u_{\sigma}^{b, n}, u_{K}^{n}\right)\right) f\left(s\left(u_{\sigma}^{b, n}, u_{K}^{n}\right)\right)+\int_{s\left(u_{\sigma}^{b, n}, u_{K}^{n}\right)}^{\kappa} \eta^{\prime \prime}(t) f(t) \mathrm{d} t \\
& \leqslant \eta^{\prime}\left(s\left(u_{\sigma}^{b, n}, u_{K}^{n}\right)\right) f\left(s\left(u_{\sigma}^{b, n}, u_{K}^{n}\right)\right)+f\left(s\left(u_{\sigma}^{b, n}, u_{K}^{n}\right)\right) \int_{s\left(u_{\sigma}^{b, n}, u_{K}^{n}\right)}^{\kappa} \eta^{\prime \prime}(t) \mathrm{d} t \\
& =0 \\
& \leqslant C_{f} \eta\left(u_{\sigma}^{b, n}\right) .
\end{aligned}
$$

Case 2: $u_{K}^{n} \leqslant u_{\sigma}^{b, n} \leqslant \kappa$

In this case, we get that $s\left(u_{K}^{n}, u_{\sigma}^{b, n}\right) \leqslant \kappa$ and $s\left(u_{\sigma}^{b, n}, u_{K}^{n}\right) \leqslant \kappa$. Similarly to Case 1 , we obtain that

$$
G\left(u_{K}^{n}, u_{\sigma}^{b, n}\right) \geqslant-C_{f} \eta\left(u_{\sigma}^{b, n}\right) \text { and } G\left(u_{\sigma}^{b, n}, u_{K}^{n}\right) \leqslant C_{f} \eta\left(u_{\sigma}^{b, n}\right) .
$$

Case 3: $\kappa \leqslant u_{K}^{n} \leqslant u_{\sigma}^{b, n}$

Since on $\left[\kappa,+\infty\left[\eta\right.\right.$ is nondecreasing and $\eta^{\prime}$ nonnegative, one gets

$$
G\left(u_{K}^{n}, u_{\sigma}^{b, n}\right)=\int_{\kappa}^{s\left(u_{K}^{n}, u_{\sigma}^{b, n}\right)} \eta^{\prime}(t) f^{\prime}(t) \mathrm{d} t \geqslant-C_{f} \int_{\kappa}^{s\left(u_{K}^{n}, u_{\sigma}^{b, n}\right)} \eta^{\prime}(t) \mathrm{d} t \geqslant-C_{f} \eta\left(u_{\sigma}^{b, n}\right) .
$$

In the same way,

$$
G\left(u_{\sigma}^{b, n}, u_{K}^{n}\right)=\int_{\kappa}^{s\left(u_{\sigma}^{b, n}, u_{K}^{n}\right)} \eta^{\prime}(t) f^{\prime}(t) \mathrm{d} t \leqslant C_{f} \int_{\kappa}^{s\left(u_{\sigma}^{b, n}, u_{K}^{n}\right)} \eta^{\prime}(t) \mathrm{d} t \leqslant C_{f} \eta\left(u_{\sigma}^{b, n}\right) .
$$

Case 4: $\kappa \leqslant u_{\sigma}^{b, n} \leqslant u_{K}^{n}$.

Since on $\left[\kappa,+\infty\left[\eta\right.\right.$ is nondecreasing and $\eta^{\prime}$ nonnegative, one gets

$$
\begin{aligned}
G\left(u_{K}^{n}, u_{\sigma}^{b, n}\right)= & \int_{\kappa}^{s\left(u_{K}^{n}, u_{\sigma}^{b, n}\right)} \eta^{\prime}(t) f^{\prime}(t) \mathrm{d} t \\
= & \int_{\kappa}^{u_{\sigma}^{b, n}} \eta^{\prime}(t) f^{\prime}(t) \mathrm{d} t+\int_{u_{\sigma}^{b, n}}^{s\left(u_{K}^{n}, u_{\sigma}^{b, n}\right)} \eta^{\prime}(t) f^{\prime}(t) \mathrm{d} t \\
\geqslant & -C_{f} \eta\left(u_{\sigma}^{b, n}\right)+\left[\eta^{\prime}(t) f(t)\right]_{u_{\sigma}^{b, n}}^{s\left(u_{K}^{n}, u_{\sigma}^{b, n}\right)}-\int_{u_{\sigma}^{b, n}}^{s\left(u_{K}^{n}, u_{\sigma}^{b, n}\right)} \eta^{\prime \prime}(t) f(t) \mathrm{d} t \\
\geqslant & -C_{f} \eta\left(u_{\sigma}^{b, n}\right)+\eta^{\prime}\left(s\left(u_{K}^{n}, u_{\sigma}^{b, n}\right)\right) f\left(s\left(u_{K}^{n}, u_{\sigma}^{b, n}\right)\right)-\eta^{\prime}\left(u_{\sigma}^{b, n}\right) f\left(u_{\sigma}^{b, n}\right) \\
& -f\left(s\left(u_{K}^{n}, u_{\sigma}^{b, n}\right)\right) \int_{u_{\sigma}^{b, n}}^{s\left(u_{K}^{n}, u_{\sigma}^{b, n}\right)} \eta^{\prime \prime}(t) \mathrm{d} t \\
\geqslant & -C_{f} \eta\left(u_{\sigma}^{b, n}\right)+\eta^{\prime}\left(u_{\sigma}^{b, n}\right)\left(f\left(s\left(u_{K}^{n}, u_{\sigma}^{b, n}\right)\right)-f\left(u_{\sigma}^{b, n}\right)\right) \\
\geqslant & -C_{f} \eta\left(u_{\sigma}^{b, n}\right) .
\end{aligned}
$$


Moreover

$$
\begin{aligned}
G\left(u_{\sigma}^{b, n}, u_{K}^{n}\right) & =\int_{\kappa}^{u_{\sigma}^{b, n}} \eta^{\prime}(t) f^{\prime}(t) \mathrm{d} t+\int_{u_{\sigma}^{b, n}}^{s\left(u_{\sigma}^{b, n}, u_{K}^{n}\right)} \eta^{\prime}(t) f^{\prime}(t) \mathrm{d} t \\
& \leqslant C_{f} \eta\left(u_{\sigma}^{b, n}\right)+\left[\eta^{\prime}(t) f(t)\right]_{u_{\sigma}^{b, n}}^{s\left(u_{\sigma}^{b, n}, u_{K}^{n}\right)}-\int_{u_{\sigma}^{b, n}}^{s\left(u_{\sigma}^{b, n}, u_{K}^{n}\right)} \eta^{\prime \prime}(t) f(t) \mathrm{d} t \\
& \leqslant C_{f} \eta\left(u_{\sigma}^{b, n}\right)+\left[\eta^{\prime}(t) f(t)\right]_{u_{\sigma}^{b, n}}^{s\left(u_{\sigma}^{b, n}, u_{K}^{n}\right)}-f\left(s\left(u_{\sigma}^{b, n}, u_{K}^{n}\right)\right) \int_{u_{\sigma}^{b, n}}^{s\left(u_{\sigma}^{b, n}, u_{K}^{n}\right)} \eta^{\prime \prime}(t) \mathrm{d} t \\
& \leqslant C_{f} \eta\left(u_{\sigma}^{b, n}\right)+\eta^{\prime}\left(u_{\sigma}^{b, n}\right)\left(f\left(s\left(u_{\sigma}^{b, n}, u_{K}^{n}\right)\right)-f\left(u_{\sigma}^{b, n}\right)\right) \\
& \leqslant C_{f} \eta\left(u_{\sigma}^{b, n}\right) .
\end{aligned}
$$

Case 5: $u_{\sigma}^{b, n} \leqslant \kappa \leqslant u_{K}^{n}$

Note that if $s\left(u_{K}^{n}, u_{\sigma}^{b, n}\right) \leqslant \kappa$, then since on $\left.]-\infty, \kappa\right] \eta^{\prime}$ is nonpositive, we get

$$
G\left(u_{K}^{n}, u_{\sigma}^{b, n}\right)=-\int_{s\left(u_{K}^{n}, u_{\sigma}^{b, n}\right)}^{\kappa} \eta^{\prime}(t) f^{\prime}(t) \mathrm{d} t \geqslant C_{f} \int_{s\left(u_{K}^{n}, u_{\sigma}^{b, n}\right)}^{\kappa} \eta^{\prime}(t) \mathrm{d} t \geqslant C_{f} \int_{u_{\sigma}^{b, n}}^{\kappa} \eta^{\prime}(t) \mathrm{d} t \geqslant-C_{f} \eta\left(u_{\sigma}^{b, n}\right) .
$$

Else, if $s\left(u_{K}^{n}, u_{\sigma}^{b, n}\right) \geqslant \kappa$, using the nonnegativity of $\eta$ we have

$$
\begin{aligned}
G\left(u_{K}^{n}, u_{\sigma}^{b, n}\right) & =\left[\eta^{\prime}(t) f(t)\right]_{\kappa}^{s\left(u_{K}^{n}, u_{\sigma}^{b, n}\right)}-\int_{\kappa}^{s\left(u_{K}^{n}, u_{\sigma}^{b, n}\right)} \eta^{\prime \prime}(t) f(t) \mathrm{d} t \\
& \geqslant \eta^{\prime}\left(s\left(u_{K}^{n}, u_{\sigma}^{b, n}\right)\right) f\left(s\left(u_{K}^{n}, u_{\sigma}^{b, n}\right)\right)-f\left(s\left(u_{K}^{n}, u_{\sigma}^{b, n}\right)\right) \int_{\kappa}^{s\left(u_{K}^{n}, u_{\sigma}^{b, n}\right)} \eta^{\prime \prime}(t) \mathrm{d} t \\
& =0 \\
& \geqslant-C_{f} \eta\left(u_{\sigma}^{b, n}\right) .
\end{aligned}
$$

Moreover, if $s\left(u_{\sigma}^{b, n}, u_{K}^{n}\right) \leqslant \kappa$, since $\eta$ is nonincreasing on ] $\left.-\infty, \kappa\right]$ one gets

$$
\begin{aligned}
G\left(u_{\sigma}^{b, n}, u_{K}^{n}\right) & =-\int_{s\left(u_{\sigma}^{b, n}, u_{K}^{n}\right)}^{\kappa} \eta^{\prime}(t) f^{\prime}(t) \mathrm{d} t \\
& \leqslant-C_{f} \int_{s\left(u_{\sigma}^{b, n}, u_{K}^{n}\right)}^{\kappa} \eta^{\prime}(t) \mathrm{d} t \\
& =C_{f} \eta\left(s\left(u_{\sigma}^{b, n}, u_{K}^{n}\right)\right) \\
& \leqslant C_{f} \eta\left(u_{\sigma}^{b, n}\right) .
\end{aligned}
$$

And in the case where $s\left(u_{\sigma}^{b, n}, u_{K}^{n}\right) \geqslant \kappa$, we have using again the nonnegativity of $\eta$

$$
\begin{aligned}
G\left(u_{\sigma}^{b, n}, u_{K}^{n}\right) & =\int_{\kappa}^{s\left(u_{\sigma}^{b, n}, u_{K}^{n}\right)} \eta^{\prime}(t) f^{\prime}(t) \mathrm{d} t \\
& \leqslant\left[\eta^{\prime}(t) f(t)\right]_{\kappa}^{s\left(u_{\sigma}^{b, n}, u_{K}^{n}\right)}-\int_{\kappa}^{s\left(u_{\sigma}^{b, n}, u_{K}^{n}\right)} \eta^{\prime \prime}(t) f(t) \mathrm{d} t \\
& \leqslant \eta^{\prime}\left(s\left(u_{\sigma}^{b, n}, u_{K}^{n}\right)\right) f\left(s\left(u_{\sigma}^{b, n}, u_{K}^{n}\right)\right)-f\left(s\left(u_{\sigma}^{b, n}, u_{K}^{n}\right)\right) \int_{\kappa}^{s\left(u_{\sigma}^{b, n}, u_{K}^{n}\right)} \eta^{\prime \prime}(t) \mathrm{d} t \\
& =0 \\
& \leqslant C_{f} \eta\left(u_{\sigma}^{b, n}\right) .
\end{aligned}
$$


Case 6: $u_{\sigma}^{b, n} \leqslant u_{K}^{n} \leqslant \kappa$

In this case, $s\left(u_{K}^{n}, u_{\sigma}^{b, n}\right) \leqslant \kappa$, using the proof of Case 5 , one gets directly

$$
G\left(u_{K}^{n}, u_{\sigma}^{b, n}\right) \geqslant-C_{f} \eta\left(u_{\sigma}^{b, n}\right) .
$$

And in the same manner, $s\left(u_{\sigma}^{b, n}, u_{K}^{n}\right) \leqslant \kappa$ so that using again Case 5 ,

$$
G\left(u_{\sigma}^{b, n}, u_{K}^{n}\right) \leqslant C_{f} \eta\left(u_{\sigma}^{b, n}\right) .
$$

Finally, we have shown, using (5.8) and (5.9) that for almost all $\omega \in \Omega$,

$$
\begin{aligned}
B^{h, k} & =B^{h, k}-B_{1}^{h, k}+B_{1}^{h, k}-B_{2}^{h, k}+B_{2}^{h, k}-B_{3}^{h, k}+B_{3}^{h, k} \\
& \geqslant B^{h, k}-B_{1}^{h, k}+B_{2, \text { int }}^{h, k}-B_{3, \text { int }}^{h, k}+\tilde{B}_{3, b}^{h, k}-B_{3, b}^{h, k}+B_{3}^{h, k} .
\end{aligned}
$$

I.3 Study of $C^{h, k}$ : we decompose $C^{h, k}$ in the following way

$$
C^{h, k}=C^{h, k}-\tilde{C}^{h, k}+\tilde{C}^{h, k},
$$

where

$$
\tilde{C}^{h, k}=\sum_{n=0}^{N-1} \sum_{K \in \mathcal{T}} \int_{K} \int_{n k}^{(n+1) k} \eta^{\prime}\left(u_{K}^{n}\right) g\left(u_{K}^{n}\right) \varphi(x, n k) \mathrm{d} W(t) \mathrm{d} x .
$$

I.4 Study of $D^{h, k}$ : we decompose $D^{h, k}$ in the following way

$$
D^{h, k}=D^{h, k}-\tilde{D}^{h, k}+\tilde{D}^{h, k},
$$

where

$$
\tilde{D}^{h, k}=\frac{1}{2} \sum_{n=0}^{N-1} \sum_{K \in \mathcal{T}} \int_{n k}^{(n+1) k} \int_{K} \eta^{\prime \prime}\left(u_{K}^{n}\right) g^{2}\left(u_{K}^{n}\right) \varphi(x, n k) \mathrm{d} x \mathrm{~d} t .
$$

Conclusion of Step I:

Since P-a.s in $\Omega, A^{h, k}=-B^{h, k}+C^{h, k}+D^{h, k}$, we get by using inequality (5.10) that

$$
\begin{aligned}
& -A^{h, k}-B_{3}^{h, k}+\tilde{C}^{h, k}+\tilde{D}^{h, k} \\
\geqslant & \left(B^{h, k}-B_{1}^{h, k}\right)+\left(B_{2, \text { int }}^{h, k}-B_{3, \text { int }}^{h, k}\right)+\left(\tilde{B}_{3, b}^{h, k}-B_{3, b}^{h, k}\right)+\left(\tilde{C}^{h, k}-C^{h, k}\right)+\left(\tilde{D}^{h, k}-D^{h, k}\right) .
\end{aligned}
$$

In this way

$$
\begin{aligned}
-\sum_{n=0}^{N-1} \sum_{K \in \mathcal{T}} & \int_{K}\left(\eta\left(u_{K}^{n+1}\right)-\eta\left(u_{K}^{n}\right)\right) \varphi(x, n k) \mathrm{d} x \\
& +\sum_{n=0}^{N-1} \sum_{K \in \mathcal{T}} \int_{n k}^{(n+1) k} \int_{K} \Phi\left(u_{K}^{n}\right) \boldsymbol{v}(x, t) . \nabla_{x} \varphi(x, n k) \mathrm{d} x \mathrm{~d} t \\
& +\sum_{n=0}^{N-1} \sum_{K \in \mathcal{T}} \int_{n k}^{(n+1) k} \int_{K} \eta^{\prime}\left(u_{K}^{n}\right) g\left(u_{K}^{n}\right) \varphi(x, n k) \mathrm{d} x \mathrm{~d} W(t) \\
& +\frac{1}{2} \sum_{n=0}^{N-1} \sum_{K \in \mathcal{T}} \int_{n k}^{(n+1) k} \int_{K} \eta^{\prime \prime}\left(u_{K}^{n}\right) g^{2}\left(u_{K}^{n}\right) \varphi(x, n k) \mathrm{d} x \mathrm{~d} t \\
& +C_{f} V \sum_{n=0}^{N-1} \sum_{\sigma \in \mathcal{E}^{b}} \int_{n k}^{(n+1) k} \int_{\sigma} \eta\left(u^{b}(x, t)\right) \varphi(x, n k) \mathrm{d} \gamma(x) \mathrm{d} t \\
\geqslant & R^{h, k},
\end{aligned}
$$


which is exactly inequality (5.5), where

$$
R^{h, k}=\left(B^{h, k}-B_{1}^{h, k}\right)+\left(B_{2, \text { int }}^{h, k}-B_{3, \text { int }}^{h, k}\right)+\left(\tilde{B}_{3, b}^{h, k}-B_{3, b}^{h, k}\right)+\left(\tilde{C}^{h, k}-C^{h, k}\right)+\left(\tilde{D}^{h, k}-D^{h, k}\right) .
$$

Step II: Convergence of $R_{h, k}$

In what follows, we consider $A$ a $P$-measurable set of $\Omega$. In this second step, we show that

$$
E\left[\mathbb{1}_{A} R_{h, k}\right] \underset{h \rightarrow 0}{\rightarrow} 0 .
$$

To do this, we show that the following quantities converge to 0 :

$$
\begin{aligned}
& E\left[\mathbb{1}_{A}\left(B^{h, k}-B_{1}^{h, k}\right)\right], E\left[\mathbb{1}_{A}\left(B_{2, i n t}^{h, k}-B_{3, \text { int }}^{h, k}\right)\right], E\left[\mathbb{1}_{A}\left(\tilde{B}_{3, b}^{h, k}-B_{3, b}^{h, k}\right)\right], E\left[\mathbb{1}_{A}\left(\tilde{C}^{h, k}-C^{h, k}\right)\right] \text { and } \\
& E\left[\mathbb{1}_{A}\left(\tilde{D}^{h, k}-D^{h, k}\right)\right] .
\end{aligned}
$$

II.1 Convergence of $E\left[\mathbb{1}_{A}\left(B^{h, k}-B_{1}^{h, k}\right)\right]$

For almost all $\omega \in \Omega, t \in(n k,(n+1) k)$, any $K \in \mathcal{T}$ and any $n \in\{0, \ldots, N-1\}$, there exists $\zeta_{K}^{n}(\omega, t) \in \mathbb{R}$ such that

$$
\eta^{\prime}\left(\bar{u}_{\mathcal{T}, k}(t)\right)-\eta^{\prime}\left(u_{K}^{n}\right)=\eta^{\prime \prime}\left(\zeta_{K}^{n}(\omega, t)\right)\left(\bar{u}_{\mathcal{T}, k}(t)-u_{K}^{n}\right)
$$

Note that $\eta^{\prime \prime}\left(\zeta_{K}^{n}(\omega, t)\right)$ is measurable with respect to $\omega$ and $t$ since it depends continuously from $\bar{u}_{\mathcal{T}, k}(t)$ and $u_{K}^{n}$. In this way, by denoting $\zeta_{K}^{n}(\omega, t)=\zeta_{K}^{n}(t)$

$$
\begin{aligned}
B^{h, k}-B_{1}^{h, k}= & \sum_{n=0}^{N-1} \sum_{K \in \mathcal{T}} \int_{n k}^{(n+1) k} \frac{1}{|K|} \int_{K}\left[\eta^{\prime}\left(\bar{u}_{\mathcal{T}, k}(t)\right)-\eta^{\prime}\left(u_{K}^{n}\right)\right] \\
& \times \sum_{\sigma \in \mathcal{E}_{K}}|\sigma|\left\{v_{K, \sigma}^{n,+} F\left(u_{K}^{n}, u_{K, \sigma}^{n}\right)-v_{K, \sigma}^{n,-} F\left(u_{K, \sigma}^{n}, u_{K}^{n}\right)\right\} \varphi(x, n k) \mathrm{d} x \mathrm{~d} t \\
= & \sum_{n=0}^{N-1} \sum_{K \in \mathcal{T}} \int_{n k}^{(n+1) k} \frac{1}{|K|} \int_{K}\left[\eta^{\prime \prime}\left(\zeta_{K}^{n}(t)\right)\left(\bar{u}_{\mathcal{T}, k}(t)-u_{K}^{n}\right)\right] \\
& \times \sum_{\sigma \in \mathcal{E}_{K}}|\sigma|\left\{v_{K, \sigma}^{n,+} F\left(u_{K}^{n}, u_{K, \sigma}^{n}\right)-v_{K, \sigma}^{n,-} F\left(u_{K, \sigma}^{n}, u_{K}^{n}\right)\right\} \varphi(x, n k) \mathrm{d} x \mathrm{~d} t \\
= & Q_{1}^{h, k}+Q_{2}^{h, k},
\end{aligned}
$$


where

$$
\begin{aligned}
Q_{1}^{h, k}= & -\sum_{n=0}^{N-1} \sum_{K \in \mathcal{T}} \int_{n k}^{(n+1) k} \frac{1}{|K|} \int_{K} \eta^{\prime \prime}\left(\zeta_{K}^{n}(t)\right) \frac{t-n k}{|K|} \varphi(x, n k) \mathrm{d} x \mathrm{~d} t \\
& \times\left(\sum_{\sigma \in \mathcal{E}_{K}}|\sigma|\left\{v_{K, \sigma}^{n,+}\left(F\left(u_{K}^{n}, u_{K, \sigma}^{n}\right)-f\left(u_{K}^{n}\right)\right)-v_{K, \sigma}^{n,-}\left(F\left(u_{K, \sigma}^{n}, u_{K}^{n}\right)-f\left(u_{K}^{n}\right)\right)\right\}\right)^{2}
\end{aligned}
$$

and

$$
\begin{aligned}
Q_{2}^{h, k}= & \sum_{n=0}^{N-1} \sum_{K \in \mathcal{T}} \int_{n k}^{(n+1) k} \frac{1}{|K|} \int_{K}\left\{\eta^{\prime \prime}\left(\zeta_{K}^{n}(t)\right) g\left(u_{K}^{n}\right)(W(t)-W(n k))\right\} \\
& \times \sum_{\sigma \in \mathcal{E}_{K}}|\sigma|\left\{v_{K, \sigma}^{n,+} F\left(u_{K}^{n}, u_{K, \sigma}^{n}\right)-v_{K, \sigma}^{n,-} F\left(u_{K, \sigma}^{n}, u_{K}^{n}\right)\right\} \varphi(x, n k) \mathrm{d} x \mathrm{~d} t .
\end{aligned}
$$

- Study of $E\left[\mathbb{1}_{A} Q_{1}^{h, k}\right]$

Note that here the assumption $k / h \rightarrow 0$ as $h \rightarrow 0$ is crucial. Similarly to the proof of (4.6), we use CauchySchwarz inequality, the consequence (3.2) of the assumptions on the mesh and finally the first estimate of Proposition 4.3 to get that

$$
\begin{aligned}
\left|E\left[\mathbb{1}_{A} Q_{1}^{h, k}\right]\right|= & \mid E\left[\mathbb{1}_{A} \sum_{n=0}^{N-1} \sum_{K \in \mathcal{T}} \int_{n k}^{(n+1) k} \frac{1}{|K|} \int_{K} \eta^{\prime \prime}\left(\zeta_{K}^{n}(t)\right) \frac{t-n k}{|K|} \varphi(x, n k) \mathrm{d} x \mathrm{~d} t\right. \\
& \left.\times\left(\sum_{\sigma \in \mathcal{E}_{K}}|\sigma|\left\{v_{K, \sigma}^{n,+}\left(F\left(u_{K}^{n}, u_{K, \sigma}^{n}\right)-f\left(u_{K}^{n}\right)\right)-v_{K, \sigma}^{n,-}\left(F\left(u_{K, \sigma}^{n}, u_{K}^{n}\right)-f\left(u_{K}^{n}\right)\right)\right\}\right)^{2}\right] \mid \\
\leqslant & \left\|\eta^{\prime \prime}\right\|_{\infty}\|\varphi\|_{\infty} \sum_{n=0}^{N-1} \sum_{K \in \mathcal{T}} \frac{k^{2}}{|K|}\left(\sum_{\sigma \in \mathcal{E}_{K}}|\sigma|\left(v_{K, \sigma}^{n,+}+v_{K, \sigma}^{n,-}\right)\right) \\
& \times E\left[\sum_{\sigma \in \mathcal{E}_{K}}|\sigma|\left\{v_{K, \sigma}^{n,+}\left(F\left(u_{K}^{n}, u_{K, \sigma}^{n}\right)-f\left(u_{K}^{n}\right)\right)^{2}+v_{K, \sigma}^{n,-}\left(F\left(u_{K, \sigma}^{n}, u_{K}^{n}\right)-f\left(u_{K}^{n}\right)\right)^{2}\right\}\right] \\
\leqslant & \left\|\eta^{\prime \prime}\right\|_{\infty}\|\varphi\|_{\infty} \sum_{n=0}^{N-1} \sum_{K \in \mathcal{T}} \frac{k^{2}}{|K|} V|\partial K| \\
& \times E\left[\sum_{\sigma \in \mathcal{E}_{K}}|\sigma|\left\{v_{K, \sigma}^{n,+}\left(F\left(u_{K}^{n}, u_{K, \sigma}^{n}\right)-f\left(u_{K}^{n}\right)\right)^{2}+v_{K, \sigma}^{n,-}\left(F\left(u_{K, \sigma}^{n}, u_{K}^{n}\right)-f\left(u_{K}^{n}\right)\right)^{2}\right\}\right] \\
\leqslant & C_{1}\left\|\eta^{\prime \prime}\right\|_{\infty}\|\varphi\|_{\infty} \frac{k}{\bar{\alpha}^{2} h} V \rightarrow 0, \text { as }\left(h, \frac{k}{h}\right) \rightarrow(0,0),
\end{aligned}
$$

where $C_{1}$ is the constant given by Proposition 4.3.

- Study of $E\left[\mathbb{1}_{A} Q_{2}^{h, k}\right]$

Let $C$ be a constant depending only on $\varphi, \eta, C_{g}, g(0),|D|, T, u_{0}, u^{b}, \xi, F_{1}, F_{2}$ whose value may change from one line to another. With the same arguments and by using additionally for any $t \geqslant n k$, the independence 
between $W(t)-W(n k)$ and any $\mathcal{F}_{n k}$-measurable process, we obtain: we get

$$
\begin{aligned}
&\left(E\left[\mathbb{1}_{A} Q_{2}^{h, k}\right]\right)^{2}=\left(E \left[\mathbb{1}_{A} \sum_{n=0}^{N-1} \sum_{K \in \mathcal{T}} \int_{n k}^{(n+1) k} \frac{1}{|K|} \int_{K}\left\{\eta^{\prime \prime}\left(\zeta_{K}^{n}\right) g\left(u_{K}^{n}\right)(W(t)-W(n k))\right\}\right.\right. \\
&\left.\left.\times \sum_{\sigma \in \mathcal{E}_{K}}|\sigma|\left\{v_{K, \sigma}^{n,+} F\left(u_{K}^{n}, u_{K, \sigma}^{n}\right)-v_{K, \sigma}^{n,-} F\left(u_{K, \sigma}^{n}, u_{K}^{n}\right)\right\} \varphi(x, n k) \mathrm{d} x \mathrm{~d} t\right]\right)^{2} \\
& \leqslant E\left[\sum_{n=0}^{N-1} \sum_{K \in \mathcal{T}} \int_{n k}^{(n+1) k} \int_{K}\left|\mathbb{1}_{A} \eta^{\prime \prime}\left(\zeta_{K}^{n}\right) g\left(u_{K}^{n}\right) \varphi(x, n k)\right|^{2} \mathrm{~d} x \mathrm{~d} t\right] \\
& \times E\left[\sum_{n=0}^{N-1} \sum_{K \in \mathcal{T}} \int_{n k}^{(n+1) k} \frac{1}{|K|}\left((W(t)-W(n k)) \sum_{\sigma \in \mathcal{E}_{K}}|\sigma|\left\{v_{K, \sigma}^{n,+} F\left(u_{K}^{n}, u_{K, \sigma}^{n}\right)-v_{K, \sigma}^{n,-} F\left(u_{K, \sigma}^{n}, u_{K}^{n}\right)\right\}\right)^{2} \mathrm{~d} t\right] \\
& \leqslant C E\left[\sum_{n=0}^{N-1} \sum_{K \in \mathcal{T}} \int_{n k}^{(n+1) k} \frac{1}{|K|}\left((W(t)-W(n k)) \sum_{\sigma \in \mathcal{E}_{K}}|\sigma|\left\{v_{K, \sigma}^{n,+} F\left(u_{K}^{n}, u_{K, \sigma}^{n}\right)-v_{K, \sigma}^{n,-} F\left(u_{K, \sigma}^{n}, u_{K}^{n}\right)\right\}\right)^{2} \mathrm{~d} t\right] \\
& \leqslant C \sum_{n=0}^{N-1} \sum_{K \in \mathcal{T}} \int_{n k}^{(n+1) k} \frac{k}{|K|} E\left[\left(\sum_{\sigma \in \mathcal{E}_{K}}|\sigma|\left\{v_{K, \sigma}^{n,+} F\left(u_{K}^{n}, u_{K, \sigma}^{n}\right)-v_{K, \sigma}^{n,-} F\left(u_{K, \sigma}^{n}, u_{K}^{n}\right)\right\}\right)^{2}\right] \mathrm{d} t \\
& \leqslant C \sum_{n=0}^{N-1} \sum_{K \in \mathcal{T}} \frac{k^{2}}{|K|} E\left[\left(\sum_{\sigma \in \mathcal{E}_{K}}|\sigma|\left\{v_{K, \sigma}^{n,+}\left(F\left(u_{K}^{n}, u_{K, \sigma}^{n}\right)-f\left(u_{K}^{n}\right)\right)-v_{K, \sigma}^{n,-}\left(F\left(u_{K, \sigma}^{n}, u_{K}^{n}\right)-f\left(u_{K}^{n}\right)\right)\right\}\right)^{2}\right] \\
& \leqslant C \sum_{n=0}^{N-1} \sum_{K \in \mathcal{T}} \frac{k^{2}}{|K|}\left(\sum_{\sigma \in \mathcal{E}_{K}}|\sigma|\left(v_{K, \sigma}^{n,+}+v_{K, \sigma}^{n,-}\right)\right) \\
& \times E\left[\sum_{\sigma \in \mathcal{E}_{K}}|\sigma|\left\{v_{K, \sigma}^{n,+}\left(F\left(u_{K}^{n}, u_{K, \sigma}^{n}\right)-f\left(u_{K}^{n}\right)\right)^{2}+v_{K, \sigma}^{n,-}\left(F\left(u_{K, \sigma}^{n}, u_{K}^{n}\right)-f\left(u_{K}^{n}\right)\right)^{2}\right\}\right] \leqslant C V \frac{k}{\bar{\alpha}^{2} h} \\
& \rightarrow 0, \text { as }\left(h, \frac{k}{h}\right) \rightarrow(0,0) .
\end{aligned}
$$

II.2 Convergence of $E\left[\mathbb{1}_{A}\left(B_{2, \text { int }}^{h, k}-B_{3, \text { int }}^{h, k}\right)\right]$

Recall that thanks to Step I, we can decompose this term as

$$
B_{2, \text { int }}^{h, k}-B_{3, \text { int }}^{h, k}=S_{2, \text { int }}^{h, k}-S_{3, \text { int }}^{h, k}-\left(T_{2, \text { int }}^{h, k}-T_{3, \text { int }}^{h, k}\right) .
$$

Let us estimate separately $\left|S_{2, \text { int }}^{h, k}-S_{3, \text { int }}^{h, k}\right|$ and $\left|T_{2, \text { int }}^{h, k}-T_{3, \text { int }}^{h, k}\right|$. Firstly, note that these quantities can be rewritten in the following way:

$$
S_{2, i n t}^{h, k}-S_{3, \text { int }}^{h, k}=S_{2, i n t}^{h, k}-\tilde{S}_{i n t}^{h, k}+\tilde{S}_{i n t}^{h, k}-S_{3, i n t}^{h, k},
$$

where

$$
\tilde{S}_{i n t}^{h, k}=\sum_{n=0}^{N-1} \sum_{(K, L) \in \mathfrak{T}_{n}} k \int_{\sigma_{K, L}}\left\{v_{K, L}^{n,+}\left(G\left(u_{K}^{n}, u_{L}^{n}\right)-\Phi\left(u_{K}^{n}\right)\right)-v_{K, L}^{n,-}\left(G\left(u_{L}^{n}, u_{K}^{n}\right)-\Phi\left(u_{K}^{n}\right)\right)\right\} \varphi(x, n k) \mathrm{d} \gamma(x) .
$$


Then,

$$
\begin{aligned}
S_{2, i n t}^{h, k}-\tilde{S}_{i n t}^{h, k}= & \sum_{n=0}^{N} \sum_{(K, L) \in \mathfrak{T}_{n}} \frac{k}{|K|}\left|\sigma_{K, L}\right|\left\{v_{K, L}^{n,+}\left(G\left(u_{K}^{n}, u_{L}^{n}\right)-\Phi\left(u_{K}^{n}\right)\right)-v_{K, L}^{n,-}\left(G\left(u_{L}^{n}, u_{K}^{n}\right)-\Phi\left(u_{K}^{n}\right)\right)\right\} \\
& \times \int_{K} \varphi(y, n k) \mathrm{d} y \\
& -\sum_{n=0}^{N-1} \sum_{(K, L) \in \mathfrak{T}_{n}} k\left\{v_{K, L}^{n,+}\left(G\left(u_{K}^{n}, u_{L}^{n}\right)-\Phi\left(u_{K}^{n}\right)\right)-v_{K, L}^{n,-}\left(G\left(u_{L}^{n}, u_{K}^{n}\right)-\Phi\left(u_{K}^{n}\right)\right)\right\} \\
& \times \int_{\sigma_{K, L}} \varphi(x, n k) \mathrm{d} \gamma(x) \\
= & \sum_{n=0}^{N-1} \sum_{(K, L) \in \mathfrak{T}_{n}} k\left|\sigma_{K, L}\right|\left\{v_{K, L}^{n,+}\left(G\left(u_{K}^{n}, u_{L}^{n}\right)-\Phi\left(u_{K}^{n}\right)\right)-v_{K, L}^{n,-}\left(G\left(u_{L}^{n}, u_{K}^{n}\right)-\Phi\left(u_{K}^{n}\right)\right)\right\} \\
& \times\left\{\frac{1}{|K|} \int_{K} \varphi(y, n k) \mathrm{d} y-\frac{1}{\left|\sigma_{K, L}\right|} \int_{\sigma_{K, L}} \varphi(x, n k) \mathrm{d} \gamma(x)\right\}
\end{aligned}
$$

and

$$
\begin{aligned}
\tilde{S}_{i n t}^{h, k}-S_{3, i n t}^{h, k}= & \sum_{n=0}^{N-1} \sum_{(K, L) \in \mathfrak{T}_{n}} k \int_{\sigma_{K, L}}\left\{v_{K, L}^{n,+}\left(G\left(u_{K}^{n}, u_{L}^{n}\right)-\Phi\left(u_{K}^{n}\right)\right)-v_{K, L}^{n,-}\left(G\left(u_{L}^{n}, u_{K}^{n}\right)-\Phi\left(u_{K}^{n}\right)\right)\right\} \\
& \times \varphi(x, n k) \mathrm{d} \gamma(x) . \\
& -\sum_{n=0}^{N} \sum_{(K, L) \in \mathfrak{T}_{n}} \int_{n k}^{(n+1) k} \int_{\sigma_{K, L}}\left\{v_{K, L}^{n,+} G\left(u_{K}^{n}, u_{L}^{n}\right)-v_{K, L}^{n,-} G\left(u_{L}^{n}, u_{K}^{n}\right)-\Phi\left(u_{K}^{n}\right) \boldsymbol{v}(x, s) \cdot n_{K, L}\right\} \\
& \times \varphi(x, n k) \mathrm{d} \gamma(x) \mathrm{d} s \\
= & \sum_{n=0}^{N-1} \sum_{(K, L) \in \mathfrak{T}_{n}} \Phi\left(u_{K}^{n}\right) \int_{n k}^{(n+1) k} \int_{\sigma_{K, L}}\left[\boldsymbol{v}(x, s) \cdot n_{K, L}-\left(v_{K, L}^{n,+}-v_{K, L}^{n,-}\right)\right] \varphi(x, n k) \mathrm{d} \gamma(x) \mathrm{d} s .
\end{aligned}
$$

In this way,

$$
\begin{aligned}
S_{2, i n t}^{h, k}-S_{3, i n t}^{h, k}= & \sum_{n=0}^{N-1} \sum_{(K, L) \in \mathfrak{T}_{n}} k\left|\sigma_{K, L}\right|\left\{v_{K, L}^{n,+}\left(G\left(u_{K}^{n}, u_{L}^{n}\right)-\Phi\left(u_{K}^{n}\right)\right)-v_{K, L}^{n,-}\left(G\left(u_{L}^{n}, u_{K}^{n}\right)-\Phi\left(u_{K}^{n}\right)\right)\right\} \\
& \times\left\{\frac{1}{|K|} \int_{K} \varphi(y, n k) \mathrm{d} y-\frac{1}{\left|\sigma_{K, L}\right|} \int_{\sigma_{K, L}} \varphi(x, n k) \mathrm{d} \gamma(x)\right\} \\
& +\sum_{n=0}^{N-1} \sum_{(K, L) \in \mathfrak{T}_{n}} \Phi\left(u_{K}^{n}\right) \int_{n k}^{(n+1) k} \int_{\sigma_{K, L}}\left[\boldsymbol{v}(x, s) \cdot n_{K, L}-\left(v_{K, L}^{n,+}-v_{K, L}^{n,-}\right)\right] \varphi(x, n k) \mathrm{d} \gamma(x) \mathrm{d} s .
\end{aligned}
$$


With a similar decomposition, we have

$$
\begin{aligned}
T_{2, i n t}^{h, k}-T_{3, \text { int }}^{h, k}= & \sum_{n=0}^{N-1} \sum_{(K, L) \in \mathfrak{T}_{n}} k\left|\sigma_{K, L}\right|\left\{v_{K, L}^{n,+}\left(G\left(u_{K}^{n}, u_{L}^{n}\right)-\Phi\left(u_{L}^{n}\right)\right)-v_{K, L}^{n,-}\left(G\left(u_{L}^{n}, u_{K}^{n}\right)-\Phi\left(u_{L}^{n}\right)\right)\right\} \\
& \times\left\{\frac{1}{|L|} \int_{K} \varphi(y, n k) \mathrm{d} y-\frac{1}{\left|\sigma_{K, L}\right|} \int_{\sigma_{K, L}} \varphi(x, n k) \mathrm{d} \gamma(x)\right\} \\
& +\sum_{n=0}^{N-1} \sum_{(K, L) \in \mathfrak{T}_{n}} \Phi\left(u_{L}^{n}\right) \int_{n k}^{(n+1) k} \int_{\sigma_{K, L}}\left[\boldsymbol{v}(x, s) \cdot n_{K, L}-\left(v_{K, L}^{n,+}-v_{K, L}^{n,-}\right)\right] \varphi(x, n k) \mathrm{d} \gamma(x) \mathrm{d} s .
\end{aligned}
$$

Secondly, in order to control $\left|S_{2, \text { int }}^{h, k}-S_{3, \text { int }}^{h, k}\right|$ and $\left|T_{2, \text { int }}^{h, k}-T_{3, \text { int }}^{h, k}\right|$, we aim to bound the following quantities:

$$
\begin{aligned}
G\left(u_{K}^{n}, u_{L}^{n}\right)-\Phi\left(u_{K}^{n}\right), G\left(u_{K}^{n}, u_{L}^{n}\right)-\Phi\left(u_{L}^{n}\right), G\left(u_{L}^{n}, u_{K}^{n}\right)-\Phi\left(u_{K}^{n}\right), G\left(u_{L}^{n}, u_{K}^{n}\right)-\Phi\left(u_{L}^{n}\right), & \\
& \frac{1}{\left|\sigma_{K, L}\right|} \int_{\sigma_{K, L}} \varphi(x, n k) \mathrm{d} \gamma(x)-\frac{1}{|K|} \int_{K} \varphi(y, n k) \mathrm{d} y,
\end{aligned}
$$

and

$$
\sum_{n=0}^{N-1} \sum_{(K, L) \in \mathfrak{T}_{n}} \Phi\left(u_{K}^{n}\right) \int_{n k}^{(n+1) k} \int_{\sigma_{K, L}}\left[\boldsymbol{v}(x, s) \cdot n_{K, L}-\left(v_{K, L}^{n,+}-v_{K, L}^{n,-}\right)\right] \varphi(x, n k) \mathrm{d} \gamma(x) \mathrm{d} s .
$$

- Study of $G\left(u_{K}^{n}, u_{L}^{n}\right)-\Phi\left(u_{K}^{n}\right)$ :

Set $(K, L) \in \mathfrak{T}_{n}$, we then have $u_{K}^{n}>u_{L}^{n}$ which implies that $f\left(s\left(u_{K}^{n}, u_{L}^{n}\right)\right)=\max _{t \in\left[u_{L}^{n}, u_{K}^{n}\right]} f(t)$ and hence

$$
\begin{aligned}
G\left(u_{K}^{n}, u_{L}^{n}\right)-\Phi\left(u_{K}^{n}\right) & =\Phi\left(s\left(u_{K}^{n}, u_{L}^{n}\right)\right)-\Phi\left(u_{K}^{n}\right) \\
& =\int_{u_{K}^{n}}^{s\left(u_{K}^{n}, u_{L}^{n}\right)} \eta^{\prime}(t) f^{\prime}(t) \mathrm{d} t .
\end{aligned}
$$

First case: $\int_{u_{K}^{n}}^{s\left(u_{K}^{n}, u_{L}^{n}\right)} \eta^{\prime}(t) f^{\prime}(t) \mathrm{d} t<0$

Using an integration by parts formula, we get with $m$ such that $f(m)=\min _{t \in\left[s\left(u_{K}^{n}, u_{L}^{n}\right), u_{K}^{n}\right]} f(t)$ :

$$
\begin{aligned}
\int_{u_{K}^{n}}^{s\left(u_{K}^{n}, u_{L}^{n}\right)} \eta^{\prime}(t) f^{\prime}(t) \mathrm{d} t & =\int_{s\left(u_{K}^{n}, u_{L}^{n}\right)}^{u_{K}^{n}} \eta^{\prime \prime}(t)(f(t)-f(m)) \mathrm{d} t-\left[\eta^{\prime}(t)(f(t)-f(m))\right]_{s\left(u_{K}^{n}, u_{L}^{n}\right)}^{u_{n}^{n}} \\
& \geqslant-\eta^{\prime}\left(u_{K}^{n}\right)\left(f\left(u_{K}^{n}\right)-f(m)\right)+\eta^{\prime}\left(s\left(u_{K}^{n}, u_{L}^{n}\right)\right)\left[f\left(s\left(u_{K}^{n}, u_{L}^{n}\right)\right)-f(m)\right] \\
& \geqslant-\left\|\eta^{\prime}\right\|_{\infty}\left|f\left(u_{K}^{n}\right)-f(m)\right|-\left\|\eta^{\prime}\right\|_{\infty}\left|f\left(s\left(u_{K}^{n}, u_{L}^{n}\right)\right)-f(m)\right| \\
& \geqslant-2\left\|\eta^{\prime}\right\|_{\infty}\left|f\left(s\left(u_{K}^{n}, u_{L}^{n}\right)\right)-f(m)\right| \\
& \geqslant-2\left\|\eta^{\prime}\right\|_{\infty} \max _{u_{L}^{n} \leqslant c \leqslant d \leqslant u_{K}^{n}}|F(d, c)-F(d, d)|,
\end{aligned}
$$

indeed, since $f\left(s\left(u_{K}^{n}, u_{L}^{n}\right)\right)=\max _{t \in\left[u_{L}^{n}, u_{K}^{n}\right]} f(t)=\max _{t \in\left[s\left(u_{K}^{n}, u_{L}^{n}\right), m\right]} f(t)=F\left(m, s\left(u_{K}^{n}, u_{L}^{n}\right)\right)$, we thus have

$$
0 \leqslant f\left(s\left(u_{K}^{n}, u_{L}^{n}\right)\right)-f(m)=F\left(m, s\left(u_{K}^{n}, u_{L}^{n}\right)\right)-F(m, m) \leqslant \max _{u_{L}^{n} \leqslant c \leqslant d \leqslant u_{K}^{n}}|F(d, c)-F(d, d)| .
$$


Second case: $\int_{u_{K}^{n}}^{s\left(u_{K}^{n}, u_{L}^{n}\right)} \eta^{\prime}(t) f^{\prime}(t) \mathrm{d} t>0$.

Similarly we have:

$$
\begin{aligned}
\int_{u_{K}^{n}}^{s\left(u_{K}^{n}, u_{L}^{n}\right)} \eta^{\prime}(t) f^{\prime}(t) \mathrm{d} t & =\int_{u_{K}^{n}}^{s\left(u_{K}^{n}, u_{L}^{n}\right)} \eta^{\prime \prime}(t)\left[f(t)-f\left(s\left(u_{K}^{n}, u_{L}^{n}\right)\right)\right] \mathrm{d} t-\left[\eta^{\prime}(t)\left(f(t)-f\left(s\left(u_{K}^{n}, u_{L}^{n}\right)\right)\right)\right]_{s\left(u_{K}^{n}, u_{L}^{n}\right)}^{u_{K}^{n}} \\
& \leqslant \eta^{\prime}\left(u_{K}^{n}\right)\left[f\left(s\left(u_{K}^{n}, u_{L}^{n}\right)\right)-f\left(u_{K}^{n}\right)\right] \\
& \leqslant\left\|\eta^{\prime}\right\|_{\infty}\left|F\left(u_{K}^{n}, u_{K}^{n}\right)-F\left(u_{K}^{n}, s\left(u_{K}^{n}, u_{L}^{n}\right)\right)\right| \\
& \leqslant\left\|\eta^{\prime}\right\|_{\infty} \max _{u_{L}^{n} \leqslant c \leqslant d \leqslant u_{K}^{n}}|F(d, c)-f(d)|
\end{aligned}
$$

since $f\left(s\left(u_{K}^{n}, u_{L}^{n}\right)\right)=\max _{t \in\left[u_{L}^{n}, u_{K}^{n}\right]} f(t)=\max _{t \in\left[s\left(u_{K}^{n}, u_{L}^{n}\right), u_{K}^{n}\right]} f(t)=F\left(u_{K}^{n}, s\left(u_{K}^{n}, u_{L}^{n}\right)\right)$.

We deduce that in both cases we have

$$
\left|G\left(u_{K}^{n}, u_{L}^{n}\right)-\Phi\left(u_{K}^{n}\right)\right| \leqslant 2\left\|\eta^{\prime}\right\|_{\infty_{u_{L}^{n} \leqslant c \leqslant d \leqslant u_{K}^{n}}}|F(d, c)-f(d)| .
$$

By using the same technics, we show that for any $(K, L) \in \mathfrak{T}_{n}$

$$
\begin{aligned}
& \left|G\left(u_{L}^{n}, u_{K}^{n}\right)-\Phi\left(u_{K}^{n}\right)\right| \leqslant 2\left\|\eta^{\prime}\right\|_{\infty} \max _{u_{L}^{n} \leqslant c \leqslant d \leqslant u_{K}^{n}}|F(c, d)-f(d)| \\
& \left|G\left(u_{K}^{n}, u_{L}^{n}\right)-\Phi\left(u_{L}^{n}\right)\right| \leqslant 2\left\|\eta^{\prime}\right\|_{\infty} \max _{u_{L}^{n} \leqslant c \leqslant d \leqslant u_{K}^{n}}|F(d, c)-f(c)| \\
& \left|G\left(u_{L}^{n}, u_{K}^{n}\right)-\Phi\left(u_{L}^{n}\right)\right| \leqslant 2\left\|\eta^{\prime}\right\|_{\infty} \max _{u_{L}^{n} \leqslant c \leqslant d \leqslant u_{K}^{n}}|F(c, d)-f(c)| .
\end{aligned}
$$

- Study of $\frac{1}{\left|\sigma_{K, L}\right|} \int_{\sigma_{K, L}} \varphi(x, n k) \mathrm{d} \gamma(x)-\frac{1}{|K|} \int_{K} \varphi(y, n k) \mathrm{d} y$

Using the regularity of $\varphi$ we get the following bound:

$$
\left|\frac{1}{\left|\sigma_{K, L}\right|} \int_{\sigma_{K, L}} \varphi(x, n k) \mathrm{d} \gamma(x)-\frac{1}{|K|} \int_{K} \varphi(y, n k) \mathrm{d} y\right| \leqslant h\left\|\nabla_{x} \varphi\right\|_{\infty} .
$$

- Study of $\sum_{n=0}^{N-1} \sum_{(K, L) \in \mathfrak{T}_{n}} \Phi\left(u_{K}^{n}\right) \int_{n k}^{(n+1) k} \int_{\sigma_{K, L}}\left[\boldsymbol{v}(x, s) \cdot n_{K, L}-\left(v_{K, L}^{n,+}-v_{K, L}^{n,-}\right)\right] \varphi(x, n k) \mathrm{d} \gamma(x) \mathrm{d} s$

First note that this term is equal to

$$
\begin{aligned}
\sum_{n=0}^{N-1} \sum_{(K, L) \in \mathfrak{T}_{n}} \Phi\left(u_{K}^{n}\right) \int_{n k}^{(n+1) k} \int_{\sigma_{K, L}}\left[\frac{1}{k\left|\sigma_{K, L}\right|} \int_{n k}^{(n+1) k} \int_{\sigma_{K, L}}\{\boldsymbol{v}(x, s)-\boldsymbol{v}(y, t)\} \cdot n_{K, L} \mathrm{~d} \gamma(y) \mathrm{d} t\right] \\
\times \varphi(x, n k) \mathrm{d} \gamma(x) \mathrm{d} s
\end{aligned}
$$

and thanks to the regularity of $\boldsymbol{v}$, we deduce that there exists a constant $c(\boldsymbol{v})$ only depending on $\boldsymbol{v}$ such that

$$
\frac{1}{k\left|\sigma_{K, L}\right|}\left|\int_{n k}^{(n+1) k} \int_{\sigma_{K, L}}\{\boldsymbol{v}(x, s)-\boldsymbol{v}(y, t)\} \cdot n_{K, L} \mathrm{~d} \gamma(y) \mathrm{d} t\right| \leqslant c(\boldsymbol{v})(h+k) .
$$


Hence by denoting $x_{\sigma}$ the center of the edge $\sigma_{K, L}$, we have

$$
\begin{aligned}
& \int_{n k}^{(n+1) k} \int_{\sigma_{K, L}}\left(\frac{1}{k\left|\sigma_{K, L}\right|} \int_{\sigma_{K, L}} \int_{n k}^{(n+1) k}\{\boldsymbol{v}(x, s)-\boldsymbol{v}(y, t)\} \cdot n_{K, L} \mathrm{~d} \gamma(y) \mathrm{d} t\right) \varphi\left(x_{\sigma}, n k\right) \mathrm{d} \gamma(x) \mathrm{d} s \\
& \quad=\varphi\left(x_{\sigma}, n k\right)\left[\int_{\sigma_{K, L}} \int_{n k}^{(n+1) k} \boldsymbol{v}(x, s) \cdot n_{K, L} \mathrm{~d} \gamma(x) \mathrm{d} s-\int_{\sigma_{K, L}} \int_{n k}^{(n+1) k} \boldsymbol{v}(y, t) \cdot n_{K, L} \mathrm{~d} \gamma(y) \mathrm{d} t\right] \\
& =0,
\end{aligned}
$$

and so

$$
\begin{aligned}
& \int_{n k}^{(n+1) k} \int_{\sigma_{K, L}}\left\{\boldsymbol{v}(x, s) \cdot n_{K, L}-\left(v_{K, L}^{n,+}-v_{K, L}^{n,-}\right)\right\} \varphi(x, n k) \mathrm{d} \gamma(x) \mathrm{d} s \\
& =\int_{n k}^{(n+1) k} \int_{\sigma_{K, L}}\left(\frac{1}{k\left|\sigma_{K, L}\right|} \int_{\sigma_{K, L}} \int_{n k}^{(n+1) k}\{\boldsymbol{v}(x, s)-\boldsymbol{v}(y, t)\} \cdot n_{K, L} \mathrm{~d} \gamma(y) \mathrm{d} t\right) \\
& \quad \times\left[\varphi(x, n k)-\varphi\left(x_{\sigma}, n k\right)\right] \mathrm{d} \gamma(x) \mathrm{d} s .
\end{aligned}
$$

Thus, we deduce from this equality, (5.19) and the CFL condition (4.17) that there exists $c(\boldsymbol{v})$ depending only on $\boldsymbol{v}$ such that

$$
\begin{aligned}
\left|\int_{n k}^{(n+1) k} \int_{\sigma_{K, L}}\left\{\boldsymbol{v}(x, s) \cdot n_{K, L}-\left(v_{K, L}^{n,+}-v_{K, L}^{n,-}\right)\right\} \varphi(x, n k) \mathrm{d} \gamma(x) \mathrm{d} s\right| & \\
& \leqslant c(\boldsymbol{v})\left\|\nabla_{x} \varphi\right\|_{\infty}\left|\sigma_{K, L}\right| k h^{2}\left(1+(1-\xi) \frac{\bar{\alpha}^{2}}{V\left(F_{1}+F_{2}\right)}\right) .
\end{aligned}
$$

In this way, there exists a constant $C$ depending only on $\boldsymbol{v}, V, \varphi, u_{0}, u^{b}, T, C_{g}, \xi, \bar{\alpha}, \eta, F_{1}, F_{2}$ and $f$, whose value may change from one line to another such that

$$
\begin{aligned}
& \left|\sum_{n=0}^{N-1} \sum_{(K, L) \in \mathfrak{T}_{n}} \Phi\left(u_{K}^{n}\right) \int_{n k}^{(n+1) k} \int_{\sigma_{K, L}}\left[\boldsymbol{v}(x, s) \cdot n_{K, L}-\left(v_{K, L}^{n,+}-v_{K, L}^{n,-}\right)\right] \varphi(x, n k) \mathrm{d} \gamma(x) \mathrm{d} s\right| \\
& \quad \leqslant C h^{2} \sum_{n=0}^{N-1} k \sum_{K \in \mathcal{T}} \sum_{L \in \mathcal{N}(K)}\left|\sigma_{K, L}\right|\left|\Phi\left(u_{K}^{n}\right)\right| \\
& \quad \leqslant C h^{2} \sum_{n=0}^{N-1} \sum_{K \in \mathcal{T}} k\left|\partial K \| u_{K}^{n}\right| \\
& \quad \leqslant \frac{C}{\bar{\alpha}^{2}} h \sum_{n=0}^{N-1} \sum_{K \in \mathcal{T}} k\left|K \| u_{K}^{n}\right| \\
& \quad \leqslant C h\left\|u_{\mathcal{T}, k}\right\|_{L^{1}(D \times(0, T)) .}
\end{aligned}
$$

Similarly we get for another generic constant $C$ independent of $k$ and $h$ that

$$
\begin{array}{r}
\left|\sum_{n=0}^{N-1} \sum_{(K, L) \in \mathfrak{T}_{n}} \Phi\left(u_{L}^{n}\right) \int_{n k}^{(n+1) k} \int_{\sigma_{K, L}}\left[\boldsymbol{v}(x, s) \cdot n_{K, L}-\left(v_{K, L}^{n,+}-v_{K, L}^{n,-}\right)\right] \varphi(x, n k) \mathrm{d} \gamma(x) \mathrm{d} s\right| \\
\leqslant C h\left\|u_{\mathcal{T}, k}\right\|_{L^{1}(D \times(0, T))} .
\end{array}
$$


We are now ready to compare $B_{2, \text { int }}^{h, k}$ to $B_{3, \text { int }}^{h, k}$. Since

$$
B_{2, \text { int }}^{h, k}-B_{3, \text { int }}^{h, k}=S_{2, \text { int }}^{h, k}-S_{3, \text { int }}^{h, k}-\left(T_{2, \text { int }}^{h, k}-T_{3, \text { int }}^{h, k}\right),
$$

using (5.14), (5.15), (5.18) and (5.20) we get for some constant $C$ independent of $k$ and $h$ that

$$
\begin{aligned}
\left|S_{2, i n t}^{h, k}-S_{3, \text { int }}^{h, k}\right| \leqslant & C \sum_{n=0}^{N-1} \sum_{(K, L) \in \mathfrak{T}_{n}} k\left|\sigma_{K, L}\right| h \\
& \times\left(v_{K, L_{u_{L}^{n} \leqslant c \leqslant d \leqslant u_{K}^{n}}^{n,+}} \max |F(d, c)-f(d)|+v_{K, L}^{n,-} \max _{u_{L}^{n} \leqslant c \leqslant d \leqslant u_{K}^{n}}|F(c, d)-f(d)|\right) \\
& +C h|| u_{\mathcal{T}, k} \|_{L^{1}(D \times(0, T))} .
\end{aligned}
$$

And using (5.16), (5.17), (5.18) and (5.21), we get similarly for some constant $C$ independent of $k$ and $h$ that

$$
\begin{aligned}
\left|T_{2, \text { int }}^{h, k}-T_{3, \text { int }}^{h, k}\right| \leqslant & C \sum_{n=0}^{N-1} \sum_{(K, L) \in \mathfrak{T}_{n}} k\left|\sigma_{K, L}\right| h \\
& \times\left(v_{K, L}^{n,+} \max _{u_{L}^{n} \leqslant c \leqslant d \leqslant u_{K}^{n}}|F(d, c)-f(c)|+v_{K, L}^{n,-} \max _{u_{L}^{n} \leqslant c \leqslant d \leqslant u_{K}^{n}}|F(c, d)-f(c)|\right) \\
& +C h|| u_{\mathcal{T}, k} \|_{L^{1}(D \times(0, T))} .
\end{aligned}
$$

Combining these two inequalities and using the second inequality of Proposition 4.3, we get for some constant $C$ depending only on $\boldsymbol{v}, V, \varphi, u_{0}, u^{b}, T, C_{g}, g(0), \xi, \bar{\alpha}, \eta, F_{1}, F_{2}, f, D$ and whose value may change from one line to another that for almost all $\omega$

$$
\begin{aligned}
\left|B_{2, i n t}^{h, k}-B_{3, \text { int }}^{h, k}\right| \leqslant & C \sum_{n=0}^{N-1} \sum_{(K, L) \in \mathfrak{T}_{n}} k\left|\sigma_{K, L}\right| h\left(v_{K, L}^{n,+}\left\{\max _{u_{L}^{n} \leqslant c \leqslant d \leqslant u_{K}^{n}}|F(d, c)-f(d)|+\max _{u_{L}^{n} \leqslant c \leqslant d \leqslant u_{K}^{n}}|F(d, c)-f(c)|\right\}\right. \\
& \left.+v_{K, L}^{n,-}\left\{\max _{u_{L}^{n} \leqslant c \leqslant d \leqslant u_{K}^{n}}|F(c, d)-f(d)|+\max _{u_{L}^{n} \leqslant c \leqslant d \leqslant u_{K}^{n}}|F(c, d)-f(c)|\right\}\right) \\
& +C h\left\|u_{\mathcal{T}, k}\right\|_{L^{1}(D \times(0, T))} \\
\leqslant & C \times C_{2} h^{1 / 2}+C h\left\|u_{\mathcal{T}, k}\right\|_{L^{1}(D \times(0, T))}
\end{aligned}
$$

where $C_{2}$ is the constant given by Proposition 4.3. Therefore, for any $P$-measurable set $A$,

$$
E\left[\mathbb{1}_{A}\left(B_{2, \text { int }}^{h, k}-B_{3, \text { int }}^{h, k}\right)\right] \underset{h \rightarrow 0}{\rightarrow} 0 .
$$


II.3 Convergence of $E\left[\mathbb{1}_{A}\left(\tilde{B}_{3, b}^{h, k}-B_{3, b}^{h, k}\right)\right]$

$$
\begin{aligned}
\left|\tilde{B}_{3, b}^{h, k}-B_{3, b}^{h, k}\right|= & \left|\sum_{n=0}^{N-1} \sum_{\sigma \in \mathcal{E}^{b}} \frac{k}{|K|}\right| \sigma \mid\left\{\left(v_{K, \sigma}^{n,+}-v_{K, \sigma}^{n,-}\right) \Phi\left(u_{K}^{n}\right)+C_{f} V \eta\left(u_{\sigma}^{b, n}\right)\right\} \int_{K} \varphi(x, n k) \mathrm{d} x \\
& -\sum_{n=0}^{N-1} \sum_{\sigma \in \mathcal{E}^{b}} \int_{n k}^{(n+1) k} \int_{\sigma} \boldsymbol{v}(y, t) \cdot n_{K, \sigma} \Phi\left(u_{K}^{n}\right) \varphi(y, n k) \mathrm{d} \gamma(y) \mathrm{d} t \\
& -C_{f} V \sum_{n=0}^{N-1} \sum_{\sigma \in \mathcal{E}^{b}} \int_{n k}^{(n+1) k} \int_{\sigma} \eta\left(u^{b}(y, t)\right) \varphi(y, n k) \mathrm{d} \gamma(y) \mathrm{d} t \mid \\
\leqslant & U_{3, b}^{h, k}+\tilde{U}_{3, b}^{h, k}
\end{aligned}
$$

where

$$
U_{3, b}^{h, k}=\left|\sum_{n=0}^{N-1} \sum_{\sigma \in \mathcal{E}^{b}} \Phi\left(u_{K}^{n}\right)\left\{\frac{k}{|K|}|\sigma|\left(v_{K, \sigma}^{n,+}-v_{K, \sigma}^{n,-}\right) \int_{K} \varphi(x, n k) \mathrm{d} x-\int_{n k}^{(n+1) k} \int_{\sigma} \boldsymbol{v}(y, t) . n_{K, \sigma} \varphi(y, n k) \mathrm{d} \gamma(y) \mathrm{d} t\right\}\right|
$$

and

$$
\tilde{U}_{3, b}^{h, k}=\left|C_{f} V \sum_{n=0}^{N-1} \sum_{\sigma \in \mathcal{E}^{b}}\left\{\frac{k}{|K|}|\sigma| \eta\left(u_{\sigma}^{b, n}\right) \int_{K} \varphi(x, n k) \mathrm{d} x-\int_{n k}^{(n+1) k} \int_{\sigma} \eta\left(u^{b}(y, t)\right) \varphi(y, n k) \mathrm{d} \gamma(y) \mathrm{d} t\right\}\right| .
$$

- Study of $U_{3, b}^{h, k}$

In what follows, $C$ is a constant depending only on $\boldsymbol{v}, T,|D|, \varphi, u_{0}, u^{b}, C_{g}, \bar{\alpha}, \eta$ and $f$, whose value may change from one line to another. For almost all $\omega \in \Omega$ we have

$$
\begin{aligned}
U_{3, b}^{h, k} & =\left|\sum_{n=0}^{N-1} \sum_{\sigma \in \mathcal{E}^{b}} \Phi\left(u_{K}^{n}\right)\left\{\frac{k}{|K|}|\sigma|\left(v_{K, \sigma}^{n,+}-v_{K, \sigma}^{n,-}\right) \int_{K} \varphi(x, n k) \mathrm{d} x-\int_{n k}^{(n+1) k} \int_{\sigma} \boldsymbol{v}(y, t) \cdot n_{K, \sigma} \varphi(y, n k) \mathrm{d} \gamma(y) \mathrm{d} t\right\}\right| \\
& =\left|\sum_{n=0}^{N-1} \sum_{\sigma \in \mathcal{E}^{b}} \Phi\left(u_{K}^{n}\right)\left\{\frac{1}{|K|} \int_{n k}^{(n+1) k} \int_{\sigma} \boldsymbol{v}(y, t) \cdot n_{K, \sigma}\left(\int_{K} \varphi(x, n k) \mathrm{d} x-|K| \varphi(y, n k)\right) \mathrm{d} \gamma(y) \mathrm{d} t\right\}\right| \\
& \leqslant V \sum_{n=0}^{N-1} \sum_{\sigma \in \mathcal{E}^{b}}\left|\Phi\left(u_{K}^{n}\right)\right| \frac{1}{|K|} \int_{n k}^{(n+1) k} \int_{\sigma} \int_{K}|\varphi(x, n k)-\varphi(y, n k)| \mathrm{d} x \mathrm{~d} \gamma(y) \mathrm{d} t \\
& \leqslant C \sum_{n=0}^{N-1} k \sum_{\sigma \in \mathcal{E}^{b}} h|\sigma|\left|u_{K}^{n}-\kappa\right| .
\end{aligned}
$$

We deduce from (3.2) and the stability estimate stated in Proposition 4.1 that

$$
\sum_{\sigma \in \mathcal{E}^{b}} h|\sigma| E\left[\left(u_{K}^{n}-\kappa\right)^{2}\right] \leqslant \frac{1}{\bar{\alpha}^{2}} \sum_{K \in \mathcal{T}}|K| E\left[\left(u_{K}^{n}-\kappa\right)^{2}\right] \leqslant \frac{2}{\bar{\alpha}^{2}}\left(\kappa^{2}|D|+\left\|u_{\mathcal{T}, k}\right\|_{L^{\infty}\left(0, T ; L^{2}(\Omega \times D)\right)}\right) .
$$


Therefore, using Cauchy-Schwarz inequality, we deduce that

$$
\begin{aligned}
E\left[\mathbb{1}_{A} U_{3, b}^{h, k}\right] & \leqslant C \sum_{n=0}^{N-1} k \sum_{\sigma \in \mathcal{E}^{b}} h|\sigma| E\left[\left|u_{K}^{n}-\kappa\right|\right] \\
& \leqslant \sum_{n=0}^{N-1} k\left(\sum_{\sigma \in \mathcal{E}^{b}} h|\sigma| E\left[\left(u_{K}^{n}-\kappa\right)^{2}\right]\right)^{\frac{1}{2}}\left(\sum_{\sigma \in \mathcal{E}^{b}} h|\sigma|\right)^{\frac{1}{2}} \\
& \leqslant C \sum_{n=0}^{N-1} k \sqrt{h} \sqrt{|\partial D|} \\
& \leqslant C \sqrt{h} \underset{h \rightarrow 0}{\rightarrow} .
\end{aligned}
$$

- Study of $\tilde{U}_{3, b}^{h, k}$

Firstly we note that for any $x \in \mathbb{R}$ we have $|\eta(x)| \leqslant\left\|\eta^{\prime}\right\|_{\infty}|x-\kappa|$. Using this inequality and (5.18), we deduce that for almost all $\omega \in \Omega$ we have

$$
\begin{aligned}
\tilde{U}_{3, b}^{h, k}= & \left|C_{f} V \sum_{n=0}^{N-1} \sum_{\sigma \in \mathcal{E}^{b}}\left\{\frac{k}{|K|}|\sigma| \eta\left(u_{\sigma}^{b, n}\right) \int_{K} \varphi(x, n k) \mathrm{d} x-\int_{n k}^{(n+1) k} \int_{\sigma} \eta\left(u^{b}(y, t)\right) \varphi(y, n k) \mathrm{d} \gamma(y) \mathrm{d} t\right\}\right| \\
= & \mid C_{f} V \sum_{n=0}^{N-1} \sum_{\sigma \in \mathcal{E}^{b}}\left\{k|\sigma| \eta\left(u_{\sigma}^{b, n}\right)\left(\frac{1}{|K|} \int_{K} \varphi(x, n k) \mathrm{d} x-\frac{1}{|\sigma|} \int_{\sigma} \varphi(y, n k) \mathrm{d} \gamma(y)+\frac{1}{|\sigma|} \int_{\sigma} \varphi(y, n k) \mathrm{d} \gamma(y)\right)\right. \\
& \left.-\int_{n k}^{(n+1) k} \int_{\sigma} \eta\left(u^{b}(y, t)\right) \varphi(y, n k) \mathrm{d} \gamma(y) \mathrm{d} t\right\} \mid \\
= & \mid C_{f} V \sum_{n=0}^{N-1} \sum_{\sigma \in \mathcal{E}^{b}}\left\{k|\sigma| \eta\left(u_{\sigma}^{b, n}\right)\left(\frac{1}{|K|} \int_{K} \varphi(x, n k) \mathrm{d} x-\frac{1}{|\sigma|} \int_{\sigma} \varphi(y, n k) \mathrm{d} \gamma(y)\right)\right. \\
& \left.+\int_{n k}^{(n+1) k} \int_{\sigma}\left(\eta\left(u_{\sigma}^{b, n}\right)-\eta\left(u^{b}(y, t)\right)\right) \varphi(y, n k) \mathrm{d} \gamma(y) \mathrm{d} t\right\} \mid \\
\leqslant & C_{f} V\left\|\eta^{\prime}\right\|_{\infty} \sum_{n=0}^{N-1} \sum_{\sigma \in \mathcal{E}^{b}}\left\{\left\|\nabla_{x} \varphi\right\|_{\infty} h k\left|\sigma\left\|u_{\sigma}^{b, n}-\kappa\left|+\|\varphi\|_{\infty} \int_{n k}^{(n+1) k} \int_{\sigma}\right| u_{\sigma}^{b, n}-u^{b}(y, t) \mid \mathrm{d} \gamma(y) \mathrm{d} t\right)\right\}\right. \\
\leqslant & C_{f} V\left\|\eta^{\prime}\right\|{ }_{\infty}\left\{h\left\|\nabla_{x} \varphi\right\|_{\infty}\left\|u^{b}-\kappa\right\|_{L^{2}((0, T) \times \partial D)}+\|\varphi\|_{\infty} \sum_{n=0}^{N-1} \sum_{\sigma \in \mathcal{E}^{b}} \int_{n k}^{(n+1) k} \int\left|u_{\sigma}^{b, n}-u^{b}(y, t)\right| \mathrm{d} \gamma(y) \mathrm{d} t\right\} \\
\rightarrow & 0 \text { as } h \rightarrow 0 .
\end{aligned}
$$

Therefore, for any $P$-measurable set $A, E\left[\mathbb{1}_{A}\left(\tilde{B}_{3, b}^{h, k}-B_{3, b}^{h, k}\right)\right] \underset{h \rightarrow 0}{\rightarrow} 0$. 
II.4 Convergence of $E\left[\mathbb{1}_{A}\left(\tilde{C}^{h, k}-C^{h, k}\right)\right]$

$$
\begin{aligned}
\left|E\left[\mathbb{1}_{A}\left(\tilde{C}^{h, k}-C^{h, k}\right)\right]\right|= & E\left[\sum_{n=0}^{N-1} \sum_{K \in \mathcal{T}} \int_{K} \mathbb{1}_{A} \int_{n k}^{(n+1) k}\left[\eta^{\prime}\left(\bar{u}_{\mathcal{T}, k}(t)\right)-\eta^{\prime}\left(u_{K}^{n}\right)\right] g\left(u_{K}^{n}\right) \varphi(x, n k) \mathrm{d} W(t) \mathrm{d} x\right] \mid \\
\leqslant & \mid E\left[\sum_{n=0}^{N-1} \sum_{K \in \mathcal{T}} \int_{K} \mathbb{1}_{A} \int_{n k}^{(n+1) k}\left[\eta^{\prime}\left(\bar{u}_{\mathcal{T}, k}(t)\right)-\eta^{\prime}\left(u_{K}^{n}\right)\right] g\left(u_{K}^{n}\right)\right. \\
& \times\{\varphi(x, n k)-\varphi(x, t)\} \mathrm{d} W(t) \mathrm{d} x] \mid \\
& +\left|E\left[\sum_{n=0}^{N-1} \sum_{K \in \mathcal{T}} \int_{K} \mathbb{1}_{A} \int_{n k}^{(n+1) k}\left[\eta^{\prime}\left(\bar{u}_{\mathcal{T}, k}(t)\right)-\eta^{\prime}\left(u_{K}^{n}\right)\right] g\left(u_{K}^{n}\right) \varphi(x, t) \mathrm{d} W(t) \mathrm{d} x\right]\right| \\
= & Y_{1}^{h, k}+Y_{2}^{h, k},
\end{aligned}
$$

where

$$
Y_{1}^{h, k}=\left|E\left[\sum_{n=0}^{N-1} \sum_{K \in \mathcal{T}} \int_{K} \mathbb{1}_{A} \int_{n k}^{(n+1) k}\left\{\eta^{\prime}\left(\bar{u}_{\mathcal{T}, k}(t)\right)-\eta^{\prime}\left(u_{K}^{n}\right)\right\} g\left(u_{K}^{n}\right)\{\varphi(x, n k)-\varphi(x, t)\} \mathrm{d} W(t) \mathrm{d} x\right]\right|
$$

and

$$
Y_{2}^{h, k}=\left|E\left[\sum_{n=0}^{N-1} \sum_{K \in \mathcal{T}} \mathbb{1}_{A} \int_{K} \int_{n k}^{(n+1) k}\left\{\eta^{\prime}\left(\bar{u}_{\mathcal{T}, k}(t)\right)-\eta^{\prime}\left(u_{K}^{n}\right)\right\} g\left(u_{K}^{n}\right) \varphi(x, t) \mathrm{d} W(t) \mathrm{d} x\right]\right|
$$

Using successively Cauchy-Schwarz inequality on $\Omega \times D$, Itô isometry (see [10]) and the constant $C_{\text {est }}$ given by Proposition 4.1 one gets

$$
\begin{aligned}
Y_{1}^{h, k} & \leqslant \sqrt{|D|} \sum_{n=0}^{N-1}\left\{\sum_{K \in \mathcal{T}} \int_{K} E\left[\left(\int_{n k}^{(n+1) k}\left\{\eta^{\prime}\left(\bar{u}_{\mathcal{T}, k}(t)\right)-\eta^{\prime}\left(u_{K}^{n}\right)\right\} g\left(u_{K}^{n}\right)\{\varphi(x, n k)-\varphi(x, t)\} \mathrm{d} W(t)\right)^{2}\right] \mathrm{d} x\right\}^{1 / 2} \\
& =\sqrt{|D|} \sum_{n=0}^{N-1}\left\{\sum_{K \in \mathcal{T}} \int_{K} \int_{n k}^{(n+1) k} E\left[\left\{\eta^{\prime}\left(\bar{u}_{\mathcal{T}, k}(t)\right)-\eta^{\prime}\left(u_{K}^{n}\right)\right\}^{2} g^{2}\left(u_{K}^{n}\right)\{\varphi(x, n k)-\varphi(x, t)\}^{2}\right] \mathrm{d} t \mathrm{~d} x\right\}^{1 / 2} \\
& \leqslant 2 k \sqrt{|D| \|} \mid \varphi_{t}\left\|_{\infty}\right\| \eta^{\prime} \|_{\infty} \sum_{n=0}^{N-1}\left\{\sum_{K \in \mathcal{T}} \int_{K} \int_{n k}^{(n+1) k} E\left[g^{2}\left(u_{K}^{n}\right)\right] \mathrm{d} t \mathrm{~d} x\right\}^{1 / 2} \\
& \leqslant 2 \sqrt{2} \sqrt{k} \sqrt{|D|}\left\|\varphi_{t}\right\|_{\infty}\left\|\eta^{\prime}\right\|_{\infty}\left\{C_{g} \sum_{n=0}^{N-1} k\left(\sum_{K \in \mathcal{T}}|K| E\left[\left(u_{K}^{n}\right)^{2}\right]\right)^{1 / 2}+T|g(0)| \sqrt{|D|}\right\} \\
& \leqslant 2 \sqrt{2} \sqrt{k} \sqrt{|D|} C_{g}\left\|\varphi_{t}\right\|_{\infty}\left\|\eta^{\prime}\right\|_{\infty}\left\{T C_{e s t}+T|g(0)| \sqrt{|D|}\right\} \\
& \rightarrow 0 \text { as }(h, k / h) \rightarrow(0,0) .
\end{aligned}
$$


Note that here assumption $H_{6}$ on the function $g$ is important:

$$
\begin{aligned}
\left(Y_{2}^{h, k}\right)^{2} & =\left|E\left[\sum_{n=0}^{N-1} \sum_{K \in \mathcal{T}} \mathbb{1}_{A} \int_{K} \int_{n k}^{(n+1) k}\left\{\eta^{\prime}\left(\bar{u}_{\mathcal{T}, k}(t)\right)-\eta^{\prime}\left(u_{K}^{n}\right)\right\} g\left(u_{K}^{n}\right) \varphi(x, t) \mathrm{d} W(t) \mathrm{d} x\right]\right|^{2} \\
& =\left|E\left[\mathbb{1}_{A} \int_{D} \int_{0}^{T}\left\{\eta^{\prime}\left(\bar{u}_{\mathcal{T}, k}\right)-\eta^{\prime}\left(u_{\mathcal{T}, k}\right)\right\} g\left(u_{\mathcal{T}, k}\right) \varphi(x, t) \mathrm{d} W(t) \mathrm{d} x\right]\right|^{2} \\
& \leqslant|D| \int_{D} E\left[\left(\int_{0}^{T}\left\{\eta^{\prime}\left(\bar{u}_{\mathcal{T}, k}\right)-\eta^{\prime}\left(u_{\mathcal{T}, k}\right)\right\} g\left(u_{\mathcal{T}, k}\right) \varphi(x, t) \mathrm{d} W(t)\right)^{2}\right] \mathrm{d} x \\
& =|D| \int_{D} \int_{0}^{T} E\left[\left\{\eta^{\prime}\left(\bar{u}_{\mathcal{T}, k}\right)-\eta^{\prime}\left(u_{\mathcal{T}, k}\right)\right\}^{2} g^{2}\left(u_{\mathcal{T}, k}\right) \varphi^{2}(x, t)\right] \mathrm{d} t \mathrm{~d} x \\
& \leqslant|D|\|\varphi\|_{\infty}^{2}\left\|\eta^{\prime \prime}\right\|_{\infty}^{2}\|g\|_{\infty}^{2}\left\|\bar{u}_{\mathcal{T}, k}-u_{\mathcal{T}, k}\right\|_{L^{2}(\Omega \times Q)}^{2} \\
& \rightarrow 0 \text { as }(h, k / h) \rightarrow(0,0), \text { using Proposition } 5.2 .
\end{aligned}
$$

In this way,

$$
E\left[\mathbb{1}_{A}\left(\tilde{C}^{h, k}-C^{h, k}\right)\right] \rightarrow 0 \text { as }(h, k / h) \rightarrow(0,0)
$$

II.5 Convergence of $E\left[\mathbb{1}_{A}\left(\tilde{D}^{h, k}-D^{h, k}\right)\right]$

Note that here, again, assumption $H_{6}$ on the function $g$ is important

$$
\begin{aligned}
\left|E\left[\mathbb{1}_{A}\left(\tilde{D}^{h, k}-D^{h, k}\right)\right]\right| & =\left|\frac{1}{2} E\left[\sum_{K \in \mathcal{T}} \sum_{n=0}^{N-1} \mathbb{1}_{A} \int_{K} \int_{n k}^{(n+1) k}\left[\eta^{\prime \prime}\left(u_{K}^{n}\right)-\eta^{\prime \prime}\left(\bar{u}_{\mathcal{T}, k}(t)\right)\right] g^{2}\left(u_{K}^{n}\right) \varphi(x, n k) \mathrm{d} x \mathrm{~d} t\right]\right| \\
& \leqslant \frac{1}{2}\|g\|_{\infty}^{2}\|\varphi\|_{\infty}\left\|\eta^{\prime \prime \prime}\right\|_{\infty}\left\|\bar{u}_{\mathcal{T}, k}-u_{\mathcal{T}, k}\right\|_{L^{1}(\Omega \times Q)} \\
& \leqslant \frac{\sqrt{T|D|}}{2}\|g\|_{\infty}^{2}\|\varphi\|_{\infty}\left\|\eta^{\prime \prime \prime}\right\|_{\infty}\left\|\bar{u}_{\mathcal{T}, k}-u_{\mathcal{T}, k}\right\|_{L^{2}(\Omega \times Q)} \\
& \rightarrow 0 \text { as }(h, k / h) \rightarrow(0,0), \text { using Proposition } 5.2 .
\end{aligned}
$$

In this way,

$$
E\left[\mathbb{1}_{A}\left(\tilde{D}^{h, k}-D^{h, k}\right)\right] \rightarrow 0 \text { as }(h, k / h) \rightarrow(0,0) .
$$

\section{Conclusion of Step II:}

By gathering the results obtained previously, one gets that for any $P$-measurable set $A$,

$$
E\left[\mathbb{1}_{A} R^{h, k}\right] \rightarrow 0 \text { as }(h, k / h) \rightarrow(0,0),
$$

which concludes the proof of the proposition.

Proposition 5.4. Proposition 5.3 holds for a general monotone flux $F$, with the same assumptions.

Proof. The proof of Proposition 5.3 holds for any monotone numerical flux $F$, except when we deal with the terms

$$
B_{1}^{h, k}-B_{2}^{h, k}, B_{2, b}^{h, k}-\tilde{B}_{3, b}^{h, k} \text { and } B_{2, \text { int }}^{h, k}-B_{3, \text { int }}^{h, k},
$$


respectively in the Steps I.2.1, I.2.2 and II.2, where we truly exploit the fact that $F$ is a Godunov numerical flux. In order to adapt these three points of the proof to the case of any monotone numerical flux $F$, we use then the decomposition given by Lemma 5.1:

$$
F(a, b)=\theta(a, b) F^{G}(a, b)+(1-\theta(a, b)) F^{L F}(a, b) .
$$

Let us begin with a definition of the entropy numerical flux $G$, which uses the above decomposition: for any $a, b \in \mathbb{R}$

$$
G(a, b)=\theta(a, b) G^{G}(a, b)+(1-\theta(a, b)) G^{L F}(a, b),
$$

where

$$
G^{G}(a, b)=\Phi(s(a, b)) \text { and } G^{L F}(a, b)=\frac{\Phi(a)+\Phi(b)}{2}-M_{F}(\eta(b)-\eta(a)),
$$

with $s(a, b) \in[\min (a, b), \max (a, b)]$ and $M_{F}=\max \left(F_{1}, F_{2}\right)$.

Now let us analyse separately

$$
B_{1}^{h, k}-B_{2}^{h, k}, B_{2, b}^{h, k}-\tilde{B}_{3, b}^{h, k} \quad \text { and } \quad B_{2, \text { int }}^{h, k}-B_{3, \text { int }}^{h, k} .
$$

- Study of $B_{1}^{h, k}-B_{2}^{h, k}$ : let us show that P-almost surely in $\Omega$,

$$
B_{1}^{h, k}-B_{2}^{h, k} \geqslant 0 .
$$

We split $B_{1}^{h, k}-B_{2}^{h, k}$ into the sum of two terms:

$$
\begin{aligned}
B_{1}^{h, k}-B_{2}^{h, k}= & \sum_{n=0}^{N-1} \sum_{K \in \mathcal{T}} \frac{k}{|K|} \sum_{\sigma \in \mathcal{E}_{K}} \theta\left(u_{K}^{n}, u_{K, \sigma}^{n}\right) \\
& \times|\sigma|\left\{v_{K, \sigma}^{n,+}\left[\eta^{\prime}\left(u_{K}^{n}\right)\left(F^{G}\left(u_{K}^{n}, u_{K, \sigma}^{n}\right)-f\left(u_{K}^{n}\right)\right)-\left(G^{G}\left(u_{K}^{n}, u_{K, \sigma}^{n}\right)-\Phi\left(u_{K}^{n}\right)\right)\right]\right. \\
& \left.-v_{K, \sigma}^{n,-}\left[\eta^{\prime}\left(u_{K}^{n}\right)\left(F^{G}\left(u_{K, \sigma}^{n}, u_{K}^{n}\right)-f\left(u_{K}^{n}\right)\right)-\left(G^{G}\left(u_{K, \sigma}^{n}, u_{K}^{n}\right)-\Phi\left(u_{K}^{n}\right)\right)\right]\right\} \int_{K} \varphi(x, n k) \mathrm{d} x \\
& +\sum_{n=0}^{N-1} \sum_{K \in \mathcal{T}} \frac{k}{|K|} \sum_{\sigma \in \mathcal{E}_{K}}\left(1-\theta\left(u_{K}^{n}, u_{K, \sigma}^{n}\right)\right)|\sigma| \\
& \times\left\{v_{K, \sigma}^{n,+}\left[\eta^{\prime}\left(u_{K}^{n}\right)\left(F^{L F}\left(u_{K}^{n}, u_{K, \sigma}^{n}\right)-f\left(u_{K}^{n}\right)\right)-\left(G^{L F}\left(u_{K}^{n}, u_{K, \sigma}^{n}\right)-\Phi\left(u_{K}^{n}\right)\right)\right]\right. \\
& \left.-v_{K, \sigma}^{n,-}\left[\eta^{\prime}\left(u_{K}^{n}\right)\left(F^{L F}\left(u_{K, \sigma}^{n}, u_{K}^{n}\right)-f\left(u_{K}^{n}\right)\right)-\left(G^{L F}\left(u_{K, \sigma}^{n}, u_{K}^{n}\right)-\Phi\left(u_{K}^{n}\right)\right)\right]\right\} \int_{K} \varphi(x, n k) \mathrm{d} x .
\end{aligned}
$$

Note that the first sum (which involves the Godunov flux) is P-almost surely nonnegative by using the same arguments as the one used in the Step I.1.2: each term of the sum was almost surely nonnegative, which remains true when we multiply each term of the sum by $\theta\left(u_{K}^{n}, u_{K, \sigma}^{n}\right)$, which is nonnegative. In order to show that the second sum (which concerns the Lax-Friedrichs flux) is also nonnegative, we write the flux $f$ as the sum of a nondecreasing function $f_{1}$ and a nonincreasing function $f_{2}$ with

$$
f_{1}(x)=\frac{f(x)}{2}+M_{F} x \quad \text { and } \quad f_{2}(x)=\frac{f(x)}{2}-M_{F} x .
$$

We can then consider the upwind schemes associated to each of these fluxes: respectively

$$
F_{1}^{L F}(a, b)=\frac{f(a)}{2}+M_{F} a \quad \text { and } \quad F_{2}^{L F}(a, b)=\frac{f(b)}{2}-M_{F} b
$$


and notice that the Lax-Friedrichs flux $F^{L F}$ is the sum of the two fluxes $F_{1}^{L F}$ and $F_{2}^{L F}$ associated to upwind schemes (in other words, the Lax-Friedrichs scheme belongs to the class of the flux-splitting schemes). We denote by $G_{1}^{L F}$ and $G_{2}^{L F}$ the associated entropy numerical fluxes:

$$
G_{1}^{L F}(a, b)=\frac{\phi(a)+M_{F} \eta(a)}{2} \quad \text { and } \quad G_{2}^{L F}(a, b)=\frac{\phi(b)-M_{F} \eta(b)}{2} .
$$

We split the second sum in the right hand side of (5.22) into two parts:

$$
\begin{aligned}
& \sum_{n=0}^{N-1} \sum_{K \in \mathcal{T}} \frac{k}{|K|} \sum_{\sigma \in \mathcal{E}_{K}}\left(1-\theta\left(u_{K}^{n}, u_{K, \sigma}^{n}\right)\right)|\sigma|\left\{v_{K, \sigma}^{n,+}\left[\eta^{\prime}\left(u_{K}^{n}\right)\left(F^{L F}\left(u_{K}^{n}, u_{K, \sigma}^{n}\right)-f\left(u_{K}^{n}\right)\right)-\left(G^{L F}\left(u_{K}^{n}, u_{K, \sigma}^{n}\right)-\Phi\left(u_{K}^{n}\right)\right)\right]\right. \\
& \left.\quad-v_{K, \sigma}^{n,-}\left[\eta^{\prime}\left(u_{K}^{n}\right)\left(F_{1}^{L F}\left(u_{K, \sigma}^{n}, u_{K}^{n}\right)-f\left(u_{K}^{n}\right)\right)-\left(G_{1}^{L F}\left(u_{K, \sigma}^{n}, u_{K}^{n}\right)-\Phi\left(u_{K}^{n}\right)\right)\right]\right\} \int_{K} \varphi(x, n k) \mathrm{d} x \\
& =\sum_{n=0}^{N-1} \sum_{K \in \mathcal{T}} \frac{k}{|K|} \sum_{\sigma \in \mathcal{E}_{K}}\left(1-\theta\left(u_{K}^{n}, u_{K, \sigma}^{n}\right)\right)|\sigma|\left\{v_{K, \sigma}^{n,+}\left[\eta^{\prime}\left(u_{K}^{n}\right)\left(F_{1}^{L F}\left(u_{K}^{n}, u_{K, \sigma}^{n}\right)-f\left(u_{K}^{n}\right)\right)-\left(G_{1}^{L F}\left(u_{K}^{n}, u_{K, \sigma}^{n}\right)-\Phi\left(u_{K}^{n}\right)\right)\right]\right. \\
& \left.\quad-v_{K, \sigma}^{n,-}\left[\eta^{\prime}\left(u_{K}^{n}\right)\left(F_{1}^{L F}\left(u_{K, \sigma}^{n}, u_{K}^{n}\right)-f\left(u_{K}^{n}\right)\right)-\left(G_{1}^{L F}\left(u_{K, \sigma}^{n}, u_{K}^{n}\right)-\Phi\left(u_{K}^{n}\right)\right)\right]\right\} \int_{K} \varphi(x, n k) \mathrm{d} x \\
& \quad+\sum_{n=0}^{N-1} \sum_{K \in \mathcal{T}} \frac{k}{|K|} \sum_{\sigma \in \mathcal{E}_{K}}\left(1-\theta\left(u_{K}^{n}, u_{K, \sigma}^{n}\right)\right)|\sigma| \\
& \quad \times\left\{v_{K, \sigma}^{n,+}\left[\eta^{\prime}\left(u_{K}^{n}\right)\left(F_{2}^{L F}\left(u_{K}^{n}, u_{K, \sigma}^{n}\right)-f\left(u_{K}^{n}\right)\right)-\left(G_{2}^{L F}\left(u_{K}^{n}, u_{K, \sigma}^{n}\right)-\Phi\left(u_{K}^{n}\right)\right)\right]\right. \\
& \left.\quad-v_{K, \sigma}^{n,-}\left[\eta^{\prime}\left(u_{K}^{n}\right)\left(F_{2}^{L F}\left(u_{K, \sigma}^{n}, u_{K}^{n}\right)-f\left(u_{K}^{n}\right)\right)-\left(G_{2}^{L F}\left(u_{K, \sigma}^{n}, u_{K}^{n}\right)-\Phi\left(u_{K}^{n}\right)\right)\right]\right\} \int_{K} \varphi(x, n k) \mathrm{d} x
\end{aligned}
$$

We note then that each of the two sums corresponds to the term appearing when we consider a monotone flux $\left(F_{1}^{L F}\right.$ or $\left.F_{2}^{L F}\right)$ and the corresponding upwind schemes, except that each term of the sum is multiplied by $\left(1-\theta\left(u_{K}^{n}, u_{K, \sigma}^{n}\right)\right)$ which is nonnegative. Such schemes are particular cases of Godunov schemes and hence it follows from the step I.1.2 that each term of each sum is almost surely nonnegative and therefore the sum is almost surely nonnegative.

- Study of $B_{2, b}^{h, k}-\tilde{B}_{3, b}^{h, k}$ : similarly, using the decomposition result given by Lemma 5.1, and the Step I.2.2 of the proof of Proposition 5.3 we show that P-almost surely in $\Omega$,

$$
B_{2, b}^{h, k}-\tilde{B}_{3, b}^{h, k} \geqslant 0
$$

- Study of $B_{2, i n t}^{h, k}-B_{3, \text { int }}^{h, k}$ : as previously, by using again the decomposition result given by Lemma 5.1, and the Step II.2 of the proof of Proposition 5.3, we show that for any P-measurable set $A$,

$$
E\left[\mathbb{1}_{A}\left(B_{2, \text { int }}^{h, k}-B_{3, \text { int }}^{h, k}\right)\right] \underset{h \rightarrow 0}{\rightarrow} 0 .
$$

The following proposition investigates the entropy inequalities which are satisfied by the approximate solution $u_{\mathcal{T}, k}$.

Proposition 5.5 (Continuous entropy inequality on the discrete solution). Assume that hypotheses $H_{1}$ to $H_{7}$ hold. Let $\mathcal{T}$ be an admissible mesh in the sense of Definition $3.1, N \in \mathbb{N}^{\star}$ and let $k=\frac{T}{N} \in \mathbb{R}_{+}^{\star}$ be the time step. 
Then, P-a.s. in $\Omega$, for any $\eta \in \mathcal{A}$ and for any $\varphi \in \mathcal{D}^{+}\left(\mathbb{R}^{d} \times[0, T)\right)$ :

$$
\begin{aligned}
\int_{D} \eta\left(u_{0}\right) & \varphi(x, 0) \mathrm{d} x+\int_{Q} \eta\left(u_{\mathcal{T}, k}\right) \varphi_{t}(x, t) \mathrm{d} x \mathrm{~d} t+\int_{Q} \Phi\left(u_{\mathcal{T}, k}\right) \boldsymbol{v}(x, t) . \nabla_{x} \varphi(x, t) \mathrm{d} x \mathrm{~d} t \\
& +\int_{0}^{T} \int_{D} \eta^{\prime}\left(u_{\mathcal{T}, k}\right) g\left(u_{\mathcal{T}, k}\right) \varphi(x, t) \mathrm{d} x \mathrm{~d} W(t)+\frac{1}{2} \int_{Q} \eta^{\prime \prime}\left(u_{\mathcal{T}, k}\right) g^{2}\left(u_{\mathcal{T}, k}\right) \varphi(x, t) \mathrm{d} x \mathrm{~d} t \\
& +C_{f} V \int_{0}^{T} \int_{\partial D} \varphi(x, t) \eta\left(u^{b}(x, t)\right) \mathrm{d} \gamma(x) \mathrm{d} t \\
\geqslant & \tilde{R}^{h, k}
\end{aligned}
$$

where for any $P$-measurable set $A, E\left[\mathbb{1}_{A} \tilde{R}^{h, k}\right] \rightarrow 0$ as $(h, k / h) \rightarrow(0,0)$.

Proof. The proof of this proposition will be separate in two steps: in a first time we will show that inequality (5.23) holds for a convenient $\tilde{R}^{h, k}$ and in a second time, we will prove that for any $P$-measurable set $A$, $E\left[\mathbb{1}_{A} \tilde{R}^{h, k}\right] \rightarrow 0$ as $(h, k / h) \rightarrow(0,0)$.

Let $T>0, u_{0} \in L^{2}(D), \mathcal{T}$ be an admissible mesh in the sense of Definition $3.1, N \in \mathbb{N}^{\star}$ and $k=\frac{T}{N} \in \mathbb{R}_{+}^{\star}$. We assume that $(h, k / h) \rightarrow(0,0)$ in this way we can suppose that the CFL Condition

$$
k \leqslant \frac{(1-\xi) \bar{\alpha}^{2} h}{\left(F_{1}+F_{2}\right) V}
$$

holds for some $\xi \in(0,1)$. In this manner, the estimates given by Propositions 4.1 and 4.3 hold. Consider $\eta \in \mathcal{A}$ and $\varphi \in \mathcal{D}^{+}\left(\mathbb{R}^{d} \times[0, T)\right)$.

Step I: Let us show that inequality (5.23) holds for a convenient $\tilde{R}^{h, k}$.

Note that the first term of inequality (5.5) given by Proposition 5.3 can be rewritten in the following way:

$$
-\sum_{n=0}^{N-1} \sum_{K \in \mathcal{T}}\left[\eta\left(u_{K}^{n+1}\right)-\eta\left(u_{K}^{n}\right)\right] \int_{K} \varphi(x, n k) \mathrm{d} x=\int_{k}^{T} \int_{D} \eta\left(u_{\mathcal{T}, k}\right) \varphi_{t}(x, t-k) \mathrm{d} x \mathrm{~d} t+\sum_{K \in \mathcal{T}} \int_{K} \eta\left(u_{K}^{0}\right) \varphi(x, 0) \mathrm{d} x .
$$

Indeed, thanks to the discrete integration by part formula

$$
\sum_{n=1}^{N-1} a_{n}\left(b_{n}-b_{n-1}\right)=a_{N} b_{N-1}-a_{0} b_{0}-\sum_{n=0}^{N-1} b_{n}\left(a_{n+1}-a_{n}\right)
$$

and by using the fact that for all $x$ in $D$ and for $k$ small enough, $\varphi(x, N k)=\varphi(x,(N-1) k)=0$ we get

$$
\begin{aligned}
\int_{k}^{T} \int_{D} \eta\left(u_{\mathcal{T}, k}\right) \varphi_{t}(x, t-k) \mathrm{d} x \mathrm{~d} t= & \sum_{n=1}^{N-1} \sum_{K \in \mathcal{T}} \int_{K} \eta\left(u_{K}^{n}\right)[\varphi(x, n k)-\varphi(x,(n-1) k)] \mathrm{d} x \\
= & -\sum_{n=0}^{N-1} \sum_{K \in \mathcal{T}} \int_{K}\left[\eta\left(u_{K}^{n+1}\right)-\eta\left(u_{K}^{n}\right)\right] \varphi(x, n k) \mathrm{d} x \\
& +\sum_{K \in \mathcal{T}} \int_{K} \eta\left(u_{K}^{N}\right) \varphi(x,(N-1) k)-\eta\left(u_{K}^{0}\right) \varphi(x, 0) \mathrm{d} x \\
= & -\sum_{n=0}^{N-1} \sum_{K \in \mathcal{T}} \int_{K}\left[\eta\left(u_{K}^{n+1}\right)-\eta\left(u_{K}^{n}\right)\right] \varphi(x, n k) \mathrm{d} x \\
& -\sum_{K \in \mathcal{T}} \int_{K} \eta\left(u_{K}^{0}\right) \varphi(x, 0) \mathrm{d} x .
\end{aligned}
$$


By denoting

$$
\begin{aligned}
C_{1}^{h, k} & =\int_{0}^{T} \int_{D} \eta^{\prime}\left(u_{\mathcal{T}, k}\right) g\left(u_{\mathcal{T}, k}\right) \varphi(x, t) \mathrm{d} x \mathrm{~d} W(t) \\
D_{1}^{h, k} & =\frac{1}{2} \int_{Q} \eta^{\prime \prime}\left(u_{\mathcal{T}, k}\right) g^{2}\left(u_{\mathcal{T}, k}\right) \varphi(x, t) \mathrm{d} x \mathrm{~d} t
\end{aligned}
$$

one gets from inequality (5.5), inequality (5.23) with $\tilde{R}^{h, k}$ defined by

$$
\begin{aligned}
\tilde{R}^{h, k}= & R^{h, k}+\int_{D} \eta\left(u_{0}\right) \varphi(x, 0) \mathrm{d} x-\sum_{K \in \mathcal{T}} \int_{K} \eta\left(u_{K}^{0}\right) \varphi(x, 0) \mathrm{d} x \\
& +\int_{Q} \eta\left(u_{\mathcal{T}, k}\right) \varphi_{t}(x, t) \mathrm{d} x \mathrm{~d} t-\int_{k}^{T} \int_{D} \eta\left(u_{\mathcal{T}, k}\right) \varphi_{t}(x, t-k) \mathrm{d} x \mathrm{~d} t \\
& +\int_{Q} \Phi\left(u_{\mathcal{T}, k}\right) \boldsymbol{v}(x, t) \cdot \nabla_{x} \varphi(x, t) \mathrm{d} x \mathrm{~d} t-\sum_{n=0}^{N-1} \sum_{K \in \mathcal{T}} \int_{n k}^{(n+1) k} \int_{K} \Phi\left(u_{K}^{n}\right) \boldsymbol{v}(x, t) . \nabla_{x} \varphi(x, n k) \mathrm{d} x \mathrm{~d} t \\
& +C_{1}^{h, k}-\sum_{K \in \mathcal{T}} \sum_{n=0}^{N-1} \int_{K} \int_{n k}^{(n+1) k} \eta^{\prime}\left(u_{K}^{n}\right) g\left(u_{K}^{n}\right) \varphi(x, n k) \mathrm{d} W(t) \mathrm{d} x \\
& +D_{1}^{h, k}-\frac{1}{2} \sum_{n=0}^{N-1} \sum_{K \in \mathcal{T}} \int_{n k}^{(n+1) k} \int_{K} \eta^{\prime \prime}\left(u_{K}^{n}\right) g^{2}\left(u_{K}^{n}\right) \varphi(x, n k) \mathrm{d} x \mathrm{~d} t \\
& +C_{f} V\left(\int_{0}^{T} \int_{\partial D} \varphi(x, t) \eta\left(u^{b}(x, t)\right) \mathrm{d} \gamma(x) \mathrm{d} t-\sum_{n=0}^{N-1} \sum_{\sigma \in \mathcal{E}^{b}} \int_{n k}^{(n+1) k} \int_{\sigma} \eta\left(u^{b}(x, t)\right) \varphi(x, n k) \mathrm{d} \gamma(x) \mathrm{d} t\right),
\end{aligned}
$$

where $R^{h, k}$ is given by (5.13) in Proposition 5.3.

Step II: Let us show that for any $P$-measurable set $A, E\left[\mathbb{1}_{A} \tilde{R}^{h, k}\right] \rightarrow 0$ as $(h, k / h) \rightarrow(0,0)$.

Thanks to Proposition 5.3, we know that $E\left[\mathbb{1}_{A} R^{h, k}\right] \rightarrow 0$ as $(h, k / h) \rightarrow(0,0)$. Then it remains to study the convergence of the following quantities:

$$
\begin{aligned}
& E\left[\mathbb{1}_{A}\left(\int_{D} \eta\left(u_{0}\right) \varphi(x, 0) \mathrm{d} x-\sum_{K \in \mathcal{T}} \int_{K} \eta\left(u_{K}^{0}\right) \varphi(x, 0) \mathrm{d} x\right)\right], \\
& E\left[\mathbb{1}_{A}\left(\int_{Q} \eta\left(u_{\mathcal{T}, k}\right) \varphi_{t}(x, t) \mathrm{d} x \mathrm{~d} t-\int_{k}^{T} \int_{D} \eta\left(u_{\mathcal{T}, k}\right) \varphi_{t}(x, t-k) \mathrm{d} x \mathrm{~d} t\right)\right], \\
& E\left[\mathbb{1}_{A}\left(\int_{Q} \Phi\left(u_{\mathcal{T}, k}\right) \boldsymbol{v}(x, t) . \nabla_{x} \varphi(x, t) \mathrm{d} x \mathrm{~d} t-\sum_{n=0}^{N-1} \sum_{K \in \mathcal{T}} \int_{n k}^{(n+1) k} \int_{K} \Phi\left(u_{K}^{n}\right) \boldsymbol{v}(x, t) . \nabla_{x} \varphi(x, n k) \mathrm{d} x \mathrm{~d} t\right)\right], \\
& E\left[\mathbb{1}_{A}\left(C_{1}^{h, k}-\sum_{K \in \mathcal{T}} \sum_{n=0}^{N-1} \int_{K} \int_{n k}^{(n+1) k} \eta^{\prime}\left(u_{K}^{n}\right) g\left(u_{K}^{n}\right) \varphi(x, n k) \mathrm{d} W(t) \mathrm{d} x\right)\right]=E\left[\mathbb{1}_{A}\left(C_{1}^{h, k}-\tilde{C}^{h, k}\right)\right], \\
& E\left[\mathbb{1}_{A}\left(D_{1}^{h, k}-\frac{1}{2} \sum_{n=0}^{N-1} \sum_{K \in \mathcal{T}} \int_{n k}^{(n+1) k} \int_{K} \eta^{\prime \prime}\left(u_{K}^{n}\right) g^{2}\left(u_{K}^{n}\right) \varphi(x, n k) \mathrm{d} x \mathrm{~d} t\right)\right]=E\left[\mathbb{1}_{A}\left(D_{1}^{h, k}-\tilde{D}^{h, k}\right)\right], \\
& E\left[\mathbb{1}_{A} C_{f} V\left(\int_{0}^{T} \int_{\partial D} \varphi(x, t) \eta\left(u^{b}(x, t)\right) \mathrm{d} \gamma(x) \mathrm{d} t-\sum_{n=0}^{N-1} \sum_{\sigma \in \mathcal{E}^{b}} \int_{n k}^{(n+1) k} \int_{\sigma} \eta\left(u^{b}(x, t)\right) \varphi(x, n k) \mathrm{d} \gamma(x) \mathrm{d} t\right)\right],
\end{aligned}
$$


where $\tilde{C}^{h, k}$ and $\tilde{D}^{h, k}$ have been defined respectively by (5.11) and (5.12) in the proof of Proposition 5.3. Let us analyze separately the convergence of these terms as $(h, k / h) \rightarrow(0,0)$.

II.1. Convergence of $E\left[\mathbb{1}_{A}\left(\int_{D} \eta\left(u_{0}\right) \varphi(x, 0) \mathrm{d} x-\sum_{K \in \mathcal{T}} \int_{K} \eta\left(u_{K}^{0}\right) \varphi(x, 0) \mathrm{d} x\right)\right]$ Since $u_{0} \in L^{1}(D)$, one shows that this term tends to 0 as $h \rightarrow 0$. Indeed we have

$$
\left[E\left[\mathbb{1}_{A}\left(\int_{D} \eta\left(u_{0}\right) \varphi(x, 0) \mathrm{d} x-\sum_{K \in \mathcal{T}} \int_{K} \eta\left(u_{K}^{0}\right) \varphi(x, 0) \mathrm{d} x\right)\right]\left|\leqslant\left\|\eta^{\prime}\right\|_{\infty}\|\varphi\|_{\infty} \int_{D}\right| u_{0}(x)-\sum_{K \in \mathcal{T}} u_{K}^{0} \mid \mathrm{d} x,\right.
$$

which goes classically to 0 when $h$ tends to 0 (see [8], p. 135 for example).

II.2. Convergence of $E\left[\mathbb{1}_{A}\left(\int_{Q} \eta\left(u_{\mathcal{T}, k}\right) \varphi_{t}(x, t) \mathrm{d} x \mathrm{~d} t-\int_{k}^{T} \int_{D} \eta\left(u_{\mathcal{T}, k}\right) \varphi_{t}(x, t-k) \mathrm{d} x \mathrm{~d} t\right)\right]$

$$
\begin{aligned}
\mid E & {\left[\int_{Q} \eta\left(u_{\mathcal{T}, k}\right) \varphi_{t}(x, t) \mathrm{d} x \mathrm{~d} t-\int_{k}^{T} \int_{D} \eta\left(u_{\mathcal{T}, k}\right) \varphi_{t}(x, t-k) \mathrm{d} x \mathrm{~d} t\right] \mid } \\
\leqslant & E\left[\int_{k}^{T} \int_{D}\left|\eta\left(u_{\mathcal{T}, k}\right) \| \varphi_{t}(x, t)-\varphi_{t}(x, t-k)\right| \mathrm{d} x \mathrm{~d} t\right]+E\left[\int_{0}^{k} \int_{D}\left|\eta\left(u_{\mathcal{T}, k}\right)\right|\left|\varphi_{t}(x, t)\right| \mathrm{d} x \mathrm{~d} t\right] \\
\leqslant & \left\|\varphi_{t t}\right\|_{\infty} k\left\|\eta^{\prime}\right\|_{\infty} \sqrt{|D| T}\left\|u_{\mathcal{T}, k}-\kappa\right\|_{L^{2}(\Omega \times Q)} \\
& +k\left\|\varphi_{t}\right\|_{\infty}\left\|\eta^{\prime}\right\|_{\infty} \sqrt{|D|}\left\|u_{\mathcal{T}, k}-\kappa\right\|_{L^{\infty}\left(0, T ; L^{2}(\Omega \times D)\right)} .
\end{aligned}
$$

We deduce easily that $E\left[\mathbb{1}_{A}\left(\int_{Q} \eta\left(u_{\mathcal{T}, k}\right) \varphi_{t}(x, t) \mathrm{d} x \mathrm{~d} t-\int_{k}^{T} \int_{D} \eta\left(u_{\mathcal{T}, k}\right) \varphi_{t}(x, t-k) \mathrm{d} x \mathrm{~d} t\right)\right] \underset{h \rightarrow 0}{\rightarrow} 0$.

II.3. Convergence of

$$
\begin{aligned}
E & {\left[\mathbb{1}_{A}\left(\int_{Q} \Phi\left(u_{\mathcal{T}, k}\right) \boldsymbol{v}(x, t) \cdot \nabla_{x} \varphi(x, t) \mathrm{d} x \mathrm{~d} t-\sum_{n=0}^{N-1} \sum_{K \in \mathcal{T}} \int_{n k}^{(n+1) k} \int_{K} \Phi\left(u_{K}^{n}\right) \boldsymbol{v}(x, t) . \nabla_{x} \varphi(x, n k) \mathrm{d} x \mathrm{~d} t\right)\right] } \\
\mid E & {\left[\mathbb{1}_{A}\left(\int_{Q} \Phi\left(u_{\mathcal{T}, k}\right) \boldsymbol{v}(x, t) . \nabla_{x} \varphi(x, t) \mathrm{d} x \mathrm{~d} t-\sum_{n=0}^{N-1} \sum_{K \in \mathcal{T}} \int_{n k}^{(n+1) k} \int_{K} \Phi\left(u_{K}^{n}\right) \boldsymbol{v}(x, t) . \nabla_{x} \varphi(x, n k) \mathrm{d} x \mathrm{~d} t\right)\right] \mid } \\
& =\left|\sum_{n=0}^{N-1} \sum_{K \in \mathcal{T}} E\left[\mathbb{1}_{A} \int_{n k}^{(n+1) k} \int_{K} \Phi\left(u_{K}^{n}\right) \boldsymbol{v}(x, t) \cdot\left[\nabla_{x} \varphi(x, t)-\nabla_{x} \varphi(x, n k)\right] \mathrm{d} x \mathrm{~d} t\right]\right| \\
& \leqslant h\left\|\Phi^{\prime}\right\|_{\infty}\left\|\nabla_{x x} \varphi\right\|_{\infty} V E\left[\int_{0}^{T} \int_{D}\left|u_{\mathcal{T}, k}-\kappa\right| \mathrm{d} x \mathrm{~d} t\right] \\
& \leqslant h\left\|\Phi^{\prime}\right\|_{\infty}\left\|\nabla_{x x} \varphi\right\|_{\infty} V \sqrt{T|D|}\left\|u_{\mathcal{T}, k}-\kappa\right\|_{L^{2}(\Omega \times Q)} \\
& \rightarrow 0
\end{aligned}
$$


II.4. Convergence of $E\left[\mathbb{1}_{A}\left(C_{1}^{h, k}-\tilde{C}^{h, k}\right)\right]$

Using Cauchy-Schwarz inequality on $\Omega \times D$ and Itô isometry one gets

$$
\begin{aligned}
\left|E\left[\mathbb{1}_{A}\left(C_{1}^{h, k}-\tilde{C}^{h, k}\right)\right]\right| & =\left|E\left[\mathbb{1}_{A} \sum_{n=0}^{N-1} \sum_{K \in \mathcal{T}} \int_{K} \int_{n k}^{(n+1) k} \eta^{\prime}\left(u_{K}^{n}\right) g\left(u_{K}^{n}\right)\{\varphi(x, n k)-\varphi(x, t)\} \mathrm{d} W(t) \mathrm{d} x\right]\right| \\
& \leqslant \sum_{n=0}^{N-1} \sqrt{|D|}\left(\sum_{K \in \mathcal{T}} \int_{K} E\left[\left(\int_{n k}^{(n+1) k} \eta^{\prime}\left(u_{K}^{n}\right) g\left(u_{K}^{n}\right)\{\varphi(x, n k)-\varphi(x, t)\} \mathrm{d} W(t)\right)^{2}\right] \mathrm{d} x\right)^{1 / 2} \\
& =\sum_{n=0}^{N-1} \sqrt{|D|}\left(\sum_{K \in \mathcal{T}} \int_{K} \int_{n k}^{(n+1) k} E\left[\left(\eta^{\prime}\left(u_{K}^{n}\right) g\left(u_{K}^{n}\right)\{\varphi(x, n k)-\varphi(x, t)\}\right)^{2}\right] \mathrm{d} t \mathrm{~d} x\right)^{1 / 2} \\
& \leqslant k \sqrt{2} \sqrt{|D|} C_{g}|| \varphi_{t}\left\|_{\infty}|| \eta^{\prime}\right\|_{\infty} \sum_{n=0}^{N-1} k\left\{|g(0)| \sqrt{|D|}+\left(\sum_{K \in \mathcal{T}}|K| E\left[\left(u_{K}^{n}\right)^{2}\right]\right)^{1 / 2}\right\} \\
& \leqslant k \sqrt{2} \sqrt{|D|} C_{g}|| \varphi_{t}\left\|_{\infty}\right\| \eta^{\prime} \|_{\infty} T\left\{|g(0)| \sqrt{|D|}+\left\|u_{\mathcal{T}, k}\right\|_{L^{\infty}\left(0, T ; L^{2}(\Omega \times Q)\right)}\right\} \\
& \rightarrow 0
\end{aligned}
$$

where we have used Proposition 4.1 to conclude.

II.5. Convergence of $E\left[\mathbb{1}_{A}\left(D_{1}^{h, k}-\tilde{D}^{h, k}\right)\right]$

$$
\begin{aligned}
\left|E\left[\mathbb{1}_{A}\left(D_{1}^{h, k}-\tilde{D}^{h, k}\right)\right]\right| & =\left|\frac{1}{2} E\left[\sum_{n=0}^{N-1} \sum_{K \in \mathcal{T}} \int_{n k}^{(n+1) k} \int_{K} \mathbb{1}_{A} \eta^{\prime \prime}\left(u_{K}^{n}\right) g^{2}\left(u_{K}^{n}\right)[\varphi(x, n k)-\varphi(x, t)] \mathrm{d} x \mathrm{~d} t\right]\right| \\
& \leqslant \frac{1}{2} k\left\|\eta^{\prime \prime}||_{\infty}|| g\right\|_{\infty}^{2}\left\|\varphi_{t}\right\|_{\infty} T|D| \\
& \underset{h \rightarrow 0}{\rightarrow \rightarrow 0} 0
\end{aligned}
$$

II.6. Convergence of

$$
\begin{aligned}
& E\left[\mathbb{1}_{A} C_{f} V\left(\int_{0}^{T} \int_{\partial D} \varphi(x, t) \eta\left(u^{b}(x, t)\right) \mathrm{d} \gamma(x) \mathrm{d} t-\sum_{n=0}^{N-1} \sum_{\sigma \in \mathcal{E}^{b}} \int_{n k}^{(n+1) k} \int_{\sigma} \eta\left(u^{b}(x, t)\right) \varphi(x, n k) \mathrm{d} \gamma(x) \mathrm{d} t\right)\right] \\
& \left.\left|E\left[\mathbb{1}_{A}\left(\int_{0}^{T} \int_{\partial D} \varphi(x, t) \eta\left(u^{b}(x, t)\right) \mathrm{d} \gamma(x) \mathrm{d} t-\sum_{n=0}^{N-1} \sum_{\sigma \in \mathcal{E}^{b}} \int_{n k}^{(n+1) k} \int_{\sigma} \eta\left(u^{b}(x, t)\right) \varphi(x, n k) \mathrm{d} \gamma(x) \mathrm{d} t\right)\right]\right|\right] \\
& \leqslant k\left\|\varphi_{t}\right\|_{\infty} \sum_{n=0}^{N-1} \sum_{\sigma \in \mathcal{E}^{b}} \int_{n k}^{(n+1) k} \int_{\sigma}\left|\eta\left(u^{b}(x, t)\right)\right| \mathrm{d} \gamma(x) \mathrm{d} t \\
& \leqslant k\left\|\varphi_{t}\right\|_{\infty}\left\|\eta^{\prime}\right\|_{\infty} T|\partial D|\left\|u^{b}-\kappa\right\|_{L^{\infty}((0, T) \times \partial D)} \\
& \underset{h \rightarrow 0}{\rightarrow} 0 .
\end{aligned}
$$

To summarize, we proved in this second step that $E\left[\mathbb{1}_{A} \tilde{R}^{h, k}\right] \rightarrow 0$ as $(h, k / h) \rightarrow(0,0)$, which concludes the proof of the proposition. 


\subsection{Proof of the convergence}

And we prove now the convergence of the finite volume approximation $u_{\mathcal{T}, k}$ to the stochastic entropy solution of Problem (1.1).

Theorem 5.6 (Convergence to the stochastic entropy solution). Assume that hypotheses $H_{1}$ to $H_{7}$ hold. Let $\mathcal{T}$ be an admissible mesh in the sense of Definition $3.1, N \in \mathbb{N}^{\star}$, let $k=\frac{T}{N} \in \mathbb{R}_{+}^{\star}$ be the time step. Let $u_{\mathcal{T}, k}$ be the finite volume approximation defined by (3.6). Then $u_{\mathcal{T}, k}$ converges in $L^{p}(\Omega \times Q)$ for any $1 \leqslant p<2$ to the unique stochastic entropy solution of (1.1) in the sense of Definition 2.1 as $(h, k / h) \rightarrow(0,0)$.

Proof. Let $\mathcal{T}$ be an admissible mesh in the sense of Definition 3.1, $N \in \mathbb{N}^{\star}$ and let $k=\frac{T}{N} \in \mathbb{R}_{+}^{\star}$ be the time step such that $k / h \rightarrow 0$ as $h \rightarrow 0$. In this way we can suppose that (at least for $h$ small enough) the CFL Condition

$$
k \leqslant \frac{(1-\xi) \bar{\alpha}^{2} h}{V\left(F_{1}+F_{2}\right)}
$$

holds for some $\xi \in(0,1)$. In this manner, the estimates given by Propositions 4.1 and 4.3 hold. Consider $A$ a $P$-measurable set, $\eta \in \mathcal{A}, \varphi \in \mathcal{D}^{+}\left(\mathbb{R}^{d} \times[0, T)\right)$.

Let us multiply Inequality (5.23) by $\mathbb{1}_{A}$ and take the expectation. This yields:

$$
\begin{aligned}
E & {\left[\mathbb{1}_{A} \int_{\mathbb{R}^{d}} \eta\left(u_{0}\right) \varphi(x, 0) \mathrm{d} x\right]+E\left[\mathbb{1}_{A} \int_{Q} \eta\left(u_{\mathcal{T}, k}\right) \varphi_{t}(x, t) \mathrm{d} x \mathrm{~d} t\right]+E\left[\mathbb{1}_{A} \int_{Q} \Phi\left(u_{\mathcal{T}, k}\right) \boldsymbol{v}(x, t) . \nabla_{x} \varphi(x, t) \mathrm{d} x \mathrm{~d} t\right] } \\
& +E\left[\mathbb{1}_{A} \int_{0}^{T} \int_{D} \eta^{\prime}\left(u_{\mathcal{T}, k}\right) g\left(u_{\mathcal{T}, k}\right) \varphi(x, t) \mathrm{d} x \mathrm{~d} W(t)\right]+\frac{1}{2} E\left[\mathbb{1}_{A} \int_{Q} \eta^{\prime \prime}\left(u_{\mathcal{T}, k}\right) g^{2}\left(u_{\mathcal{T}, k}\right) \varphi(x, t) \mathrm{d} x \mathrm{~d} t\right] \\
& +E\left[\mathbb{1}_{A} C_{f} V \int_{0}^{T} \int_{\partial D} \varphi(x, t) \eta\left(u^{b}(x, t)\right) \mathrm{d} \gamma(x) \mathrm{d} t\right] \\
\geqslant & E\left[\mathbb{1}_{A} \tilde{R}^{h, k}\right] .
\end{aligned}
$$

To show the convergence of $u_{\mathcal{T}, k}$ towards the unique stochastic entropy solution of our problem, we aim to pass to the limit in the above inequality. Thanks to Proposition 5.5 we know that for any $P$-measurable set $A$, $E\left[\mathbb{1}_{A} \tilde{R}^{h, k}\right] \rightarrow 0$ as $(h, k / h) \rightarrow(0,0)$. Thus it remains to study the convergence of the left-hand side of $(5.24)$. Recall that thanks to the estimate stated in Proposition 4.1, $u_{\mathcal{T}, k}$ converges (up to a subsequence denoted in the same way) in the sense of Young measures to an "entropy process" denoted by $\mathbf{u}$ in $L^{2}(\Omega \times Q \times(0,1))$ (see Sect. 4.3).

1. Study of $E\left[\mathbb{1}_{A} \int_{Q} \eta\left(u_{\mathcal{T}, k}\right) \varphi_{t}(x, t) \mathrm{d} x \mathrm{~d} t\right]$

Note that $\Psi:(\omega, x, t, \nu) \in \Omega \times Q \times \mathbb{R} \mapsto \mathbb{1}_{A}(\omega) \eta(\nu) \varphi_{t}(x, t) \in \mathbb{R}$ is a Carathéodory function such that $\Psi\left(., u_{\mathcal{T}, k}\right)$ is bounded in $L^{2}(\Omega \times Q)$, it is therefore uniformly integrable, thus

$$
E\left[\mathbb{1}_{A} \int_{Q} \eta\left(u_{\mathcal{T}, k}(x, t)\right) \varphi_{t}(x, t) \mathrm{d} x \mathrm{~d} t\right] \rightarrow E\left[\mathbb{1}_{A} \int_{Q} \int_{0}^{1} \eta(\mathbf{u}(x, t, \alpha)) \mathrm{d} \alpha \varphi_{t}(x, t) \mathrm{d} x \mathrm{~d} t\right] \text { as } h \rightarrow 0 .
$$

2. Study of $E\left[\mathbb{1}_{A} \int_{Q} \Phi\left(u_{\mathcal{T}, k}\right) \boldsymbol{v}(x, t) . \nabla_{x} \varphi(x, t) \mathrm{d} x \mathrm{~d} t\right]$

Since $\Phi\left(u_{\mathcal{T}, k}\right)$ is bounded in $L^{2}(\Omega \times Q)$, using the same arguments as previously, we obtain

$$
E\left[\mathbb{1}_{A} \int_{Q} \Phi\left(u_{\mathcal{T}, k}\right) \boldsymbol{v}(x, t) \cdot \nabla_{x} \varphi(x, t) \mathrm{d} x \mathrm{~d} t\right] \rightarrow E\left[\mathbb{1}_{A} \int_{Q} \int_{0}^{1} \Phi(\mathbf{u}(x, t, \alpha)) \boldsymbol{v}(x, t) . \nabla_{x} \varphi(x, t) \mathrm{d} \alpha \mathrm{d} x \mathrm{~d} t\right] \text { as } h \rightarrow 0 .
$$


3. Study of $E\left[\mathbb{1}_{A} \int_{0}^{T} \int_{\mathbb{R}^{d}} \eta^{\prime}\left(u_{\mathcal{T}, k}\right) g\left(u_{\mathcal{T}, k}\right) \varphi(x, t) \mathrm{d} x \mathrm{~d} W(t)\right]$

By denoting $\Psi:(\omega, x, t, \nu) \in \Omega \times Q \times \mathbb{R} \mapsto \eta^{\prime}(\nu) g(\nu) \varphi(x, t) \in \mathbb{R}, \Psi\left(., u_{\mathcal{T}, k}\right)$ is bounded in $L^{2}(\Omega \times Q)$, and therefore $\Psi\left(., u_{\mathcal{T}, k}\right)$ converges weakly (up to a subsequence denoted in the same way) in $L^{2}(\Omega \times Q)$ to an element called $\chi$.

But, for any $\phi \in L^{2}(\Omega \times Q),(\omega, x, t, \nu) \in \Omega \times Q \times \mathbb{R} \mapsto \phi(\omega, x, t) \Psi(\omega, x, t, \nu)$ is a Carathéodory function such that $\left(\phi \Psi\left(., u_{\mathcal{T}, k}\right)\right)$ is uniformly integrable. It is based on the fact that for any subset $H$ of $\Omega \times Q$,

$$
\int_{H}\left|\phi \Psi\left(., u_{\mathcal{T}, k}\right)\right| \mathrm{d} x \mathrm{~d} t \mathrm{~d} P \leqslant\left\|\Psi\left(., u_{\mathcal{T}, k}\right)\right\|_{L^{2}(H)}\left[\int_{H}|\phi|^{2} \mathrm{~d} x \mathrm{~d} t \mathrm{~d} P\right]^{1 / 2} .
$$

Thus, at the limit,

$$
\int_{\Omega \times Q} \chi \phi \mathrm{d} x \mathrm{~d} t \mathrm{~d} P=\int_{\Omega \times Q} \int_{0}^{1} \Psi(., \mathbf{u}(., \alpha)) \mathrm{d} \alpha \phi \mathrm{d} x \mathrm{~d} t \mathrm{~d} P .
$$

By identification, $\Psi\left(., u_{\mathcal{T}, k}\right) \rightarrow \int_{0}^{1} \Psi(., \mathbf{u}(., \alpha)) \mathrm{d} \alpha$ weakly in $L^{2}(\Omega \times Q)$. Using now the linear continuity of the stochastic integral from $L^{2}(\Omega \times Q)$ to $L^{2}(\Omega \times D)$, which implies the continuity for the weak topology:

$$
\int_{0}^{T} \eta^{\prime}\left(u_{\mathcal{T}, k}\right) g\left(u_{\mathcal{T}, k}\right) \varphi \mathrm{d} W(t) \rightarrow \int_{0}^{T} \int_{0}^{1} \eta^{\prime}(\mathbf{u}(., \alpha)) g(\mathbf{u}(., \alpha)) \varphi \mathrm{d} \alpha \mathrm{d} W(t) \text { weakly in } L^{2}(\Omega \times D) .
$$

As $\mathbb{1}_{A} \in L^{2}(\Omega \times D)$ one gets at the limit

$$
E\left[\mathbb{1}_{A} \int_{0}^{T} \int_{D} \eta^{\prime}\left(u_{\mathcal{T}, k}\right) g\left(u_{\mathcal{T}, k}\right) \varphi(x, t) \mathrm{d} x \mathrm{~d} W(t)\right] \rightarrow E\left[\mathbb{1}_{A} \int_{0}^{T} \int_{D} \int_{0}^{1} \eta^{\prime}(\mathbf{u}(x, t, \alpha)) g(\mathbf{u}(x, t, \alpha)) \varphi(x, t) \mathrm{d} \alpha \mathrm{d} x \mathrm{~d} W(t)\right] .
$$

4. Study of $\frac{1}{2} E\left[\mathbb{1}_{A} \int_{Q} \eta^{\prime \prime}\left(u_{\mathcal{T}, k}\right) g^{2}\left(u_{\mathcal{T}, k}\right) \varphi(x, t) \mathrm{d} x \mathrm{~d} t\right]$

Since $\Psi:(\omega, x, t, \nu) \in \Omega \times Q \times \mathbb{R} \mapsto \eta^{\prime \prime}(\nu) g^{2}(\nu) \varphi(x, t) \mathbb{1}_{A}(\omega) \in \mathbb{R}$ is a Carathéodory function such that $\Psi\left(., u_{\mathcal{T}, k}\right)$ is bounded in $L^{2}(\Omega \times Q)$, at the limit we get:

$$
\frac{1}{2} E\left[\mathbb{1}_{A} \int_{Q} \eta^{\prime \prime}\left(u_{\mathcal{T}, k}\right) g^{2}\left(u_{\mathcal{T}, k}\right) \varphi(x, t) \mathrm{d} x \mathrm{~d} t\right] \rightarrow \frac{1}{2} E\left[\mathbb{1}_{A} \int_{Q} \int_{0}^{1} \eta^{\prime \prime}(\mathbf{u}(x, t, \alpha)) g^{2}(\mathbf{u}(x, t, \alpha)) \varphi(x, t) \mathrm{d} \alpha \mathrm{d} x \mathrm{~d} t\right] .
$$

Finally, by passing to the limit in Inequality (5.24), we obtain:

For any $P$-measurable set $A$, for any $\eta \in \mathcal{A}$ and for any $\varphi \in \mathcal{D}^{+}\left(\mathbb{R}^{d} \times[0, T)\right)$

$$
\begin{aligned}
0 \leqslant & E\left[\mathbb{1}_{A} \int_{\mathbb{R}^{d}} \eta\left(u_{0}\right) \varphi(x, 0) \mathrm{d} x\right]+E\left[\mathbb{1}_{A} \int_{Q} \int_{0}^{1} \eta(\mathbf{u}(x, t, \alpha)) \varphi_{t}(x, t) \mathrm{d} \alpha \mathrm{d} x \mathrm{~d} t\right] \\
& +E\left[\mathbb{1}_{A} \int_{Q} \int_{0}^{1} \Phi(\mathbf{u}(x, t, \alpha)) \boldsymbol{v}(x, t) . \nabla_{x} \varphi(x, t) \mathrm{d} \alpha \mathrm{d} x \mathrm{~d} t\right] \\
& +E\left[\mathbb{1}_{A} \int_{0}^{T} \int_{\mathbb{R}^{d}} \int_{0}^{1} \eta^{\prime}(\mathbf{u}(x, t, \alpha)) g(\mathbf{u}(x, t, \alpha)) \varphi(x, t) \mathrm{d} \alpha \mathrm{d} x \mathrm{~d} W(t)\right] \\
& +\frac{1}{2} E\left[\mathbb{1}_{A} \int_{Q} \int_{0}^{1} \eta^{\prime \prime}(\mathbf{u}(x, t, \alpha)) g^{2}(\mathbf{u}(x, t, \alpha)) \varphi(x, t) \mathrm{d} \alpha \mathrm{d} x \mathrm{~d} t\right] \\
& +E\left[\mathbb{1}_{A} C_{f} V \int_{0}^{T} \int_{\partial D} \varphi(x, t) \eta\left(u^{b}(x, t)\right) \mathrm{d} \gamma(x) \mathrm{d} t\right] .
\end{aligned}
$$

Hence $\mathbf{u}$ is a measure-valued entropy solution in the sense of Definition 2.2. Thanks to Theorem 2.3, $\mathbf{u}$ is independent of $\alpha$ and is hence the unique stochastic entropy solution in the sense of Definition 2.1 and we denote it by $u$. In this way, all the sequence of approximate solution $u_{\mathcal{T}, k}$ converges to $u$ in $L^{1}(\Omega \times Q)$. In addition, since $u_{\mathcal{T}, k}$ is bounded in $L^{2}(\Omega \times Q)$, all the sequence converges in $L^{p}(\Omega \times Q)$ for any $1 \leqslant p<2$. 


\section{REFERENCES}

[1] E.J. Balder, Lectures on Young measure theory and its applications in economics. Workshop on Measure Theory and Real Analysis (Italian), Grado (1997). Rend. Istit. Mat. Univ. Trieste 31 (2000) 1-69.

[2] C. Bauzet, On a time-splitting method for a scalar conservation law with a multiplicative stochastic perturbation and numerical experiments. J. Evol. Equ. 14 (2014) 333-356.

[3] C. Bauzet, G. Vallet and P. Wittbold, The Cauchy problem for a conservation law with a multiplicative stochastic perturbation. J. Hyperbolic Differ. Eq. 9 (2012) 661-709.

[4] C. Bauzet, J. Charrier and T. Gallouët, Convergence of flux-splitting finite volume schemes for hyperbolic scalar conservation laws with a multiplicative stochastic perturbation. Math. Comp. 85 (2016) 2777-2813.

[5] C. Bauzet, J. Charrier and T. Gallouët, Convergence of monotone finite volume schemes for hyperbolic scalar conservation laws with a multiplicative noise. Stoch. Partial Differ. Eq. Anal. Comput. 4 (2016) 150-223.

[6] C. Bauzet, G. Vallet and P. Wittbold, The Dirichlet problem for a conservation law with a multiplicative stochastic perturbation. J. Funct. Anal. 4 (2014) 2503-2545.

[7] I.H. Biswas and A.K. Majee, Stochastic conservation laws: Weak-in-time formulation and strong entropy condition. J. Funct. Anal. 7 (2014) 2199-2252.

[8] C. Chainais-Hillairet, Second-order finite-volume schemes for a non-linear hyperbolic equation: error estimate. Math. Methods Appl. Sci. 23 (2000) 467-490.

[9] G.-Q. Chen, Q. Ding and K.H. Karlsen, On nonlinear stochastic balance laws. Arch. Ration. Mech. Anal. 204 (2012) $707-743$.

[10] G. Da Prato and J. Zabczyk, Stochastic equations in infinite dimensions. Vol. 44 of Encycl. Math. Appl. Cambridge University Press, Cambridge (1992).

[11] A. Debussche and J. Vovelle, Scalar conservation laws with stochastic forcing. J. Funct. Anal. 259 (2010) $1014-1042$.

[12] R. Eymard, T. Gallouët and R. Herbin, Existence and uniqueness of the entropy solution to a nonlinear hyperbolic equation. Chinese Ann. Math. Ser. B 16 (1995) 1-14. A Chinese summary appears in Chinese Ann. Math. Ser. A 16 (1995) 119.

[13] R. Eymard, T. Gallouët and R. Herbin, Finite volume methods. Vol. VII of Handb. Numer. Anal. North-Holland, Amsterdam (2000) 713-1020.

[14] J. Feng and D. Nualart, Stochastic scalar conservation laws. J. Funct. Anal. 255 (2008) 313-373.

[15] M. Hofmanová, Bhatnagar-gross-krook approximation to stochastic scalar conservation laws. Ann. Inst. Henri Poincaré Probab. Statist. (2014).

[16] H. Holden and N.H. Risebro, A stochastic approach to conservation laws. In Third International Conference on Hyperbolic Problems. Vols. I, II (Uppsala, 1990). Studentlitteratur, Lund (1991) 575-587.

[17] K. Kobayasi and D. Noboriguchi, A stochastic conservation law with nonhomogeneous Dirichlet boundary conditions. Acta Math. Vietnam. 41 (2016) 607-632.

[18] I. Kröker and C. Rohde, Finite volume schemes for hyperbolic balance laws with multiplicative noise. Appl. Numer. Math. 62 (2012) 441-456.

[19] F. Otto, Initial-boundary value problem for a scalar conservation law. C. R. Acad. Sci. Paris Sér. I Math. 322 (1996) $729-734$.

[20] E. Yu. Panov, On measure-valued solutions of the Cauchy problem for a first-order quasilinear equation. Izv. Ross. Akad. Nauk Ser. Mat. 60 (1996) 107-148.

[21] G. Vallet, Stochastic perturbation of nonlinear degenerate parabolic problems. Differ. Integral Eq. 21 (2008) $1055-1082$.

[22] J. Vovelle, Convergence of finite volume monotone schemes for scalar conservation laws on bounded domains. Numer. Math. 90 (2002) 563-596. 